\title{
Selective ortho Thiolation Enabled by Tuning the Ancillary Ligand in Palla- dium/Norbornene Catalysis
}

\author{
Wenqiang Cai and Zhenhua Gu* \\ Department of Chemistry, Center for Excellence in Molecular Synthesis, and Hefei National La- \\ boratory for Physical Sciences at the Microscale, University of Science and Technology of China, 96 \\ Jinzhai Road, Hefei, Anhui 230026, P.R. China
}

zhgu@ustc.edu.cn

\section{General Information}

Nuclear magnetic resonances were recorded on Bruker-400 MHz instruments. Reference values for residual solvents were taken as $\delta=7.26 \mathrm{ppm}\left(\mathrm{CDCl}_{3}\right)$ for ${ }^{1} \mathrm{H} \mathrm{NMR} ; \delta=77.00 \mathrm{ppm}\left(\mathrm{CDCl}_{3}\right)$ for ${ }^{13} \mathrm{C}$ NMR. High resolution mass spectral analysis (HRMS) was performed on Waters XEVO G2 QTOF (Waters Corporation). All reactions were performed under an inert atmosphere of dry nitrogen in flame-dried glassware, unless otherwise stated. Tetrahydrofuran was distilled over sodium in the presence of benzophenone under nitrogen atmosphere. 1,4-dioxane and acetonitrile are super dry solvent with molecular sieves. Toluene, dichloromethane, 1,2-dichloroethane and N,N-dimethylformamide were distilled over calcium hydride under an atmosphere of nitrogen.

\section{General Procedure for the Synthesis of Thiosulfonates}

Preparation of the symmetrical thiosulfonate 2 a via reductive coupling of sulfonyl chloride ${ }^{l}$

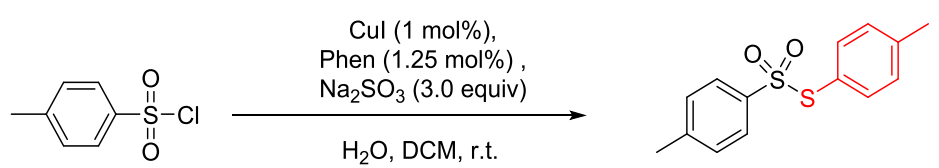

$\mathbf{2 a}$

To a solution of tosyl chloride (7.63 g, $40.0 \mathrm{mmol})$, sodium sulfite $(15.12 \mathrm{~g}, 120 \mathrm{mmol})$ and $\mathrm{H}_{2} \mathrm{O}$ $(0.72 \mathrm{~mL})$ in DCM $(100 \mathrm{~mL})$ were added copper(I) iodide $(76.2 \mathrm{mg}, 0.40 \mathrm{mmol}, 1.0 \mathrm{~mol} \%)$ and 1,10-Phenanthrolin ( $90.1 \mathrm{mg}, 0.5 \mathrm{mmol}, 1.25 \mathrm{~mol} \%)$ ), the mixture was stirred at room temperature for $4 \mathrm{~h}$ (monitored by TLC). The mixture was diluted with $\mathrm{CH}_{2} \mathrm{Cl}_{2}$, and the resulting mixture was washed with water and the organic layer was dried over $\mathrm{Na}_{2} \mathrm{SO}_{4}$. After filtration, the solvent was removed and the residue was purified by column chromatography on silica gel (50/50 DCM/hexanes) to give $\mathbf{2 a}(4.45 \mathrm{~g}, 80 \%)$ as a white solid. 
A typical procedure for synthesis of unsymmetrical thiosulfonate $2 \boldsymbol{e}$ from arenesulfinate salt and disulfide ${ }^{2}$

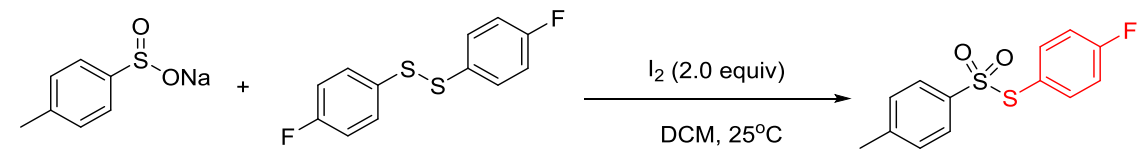

(3.2 equiv)

$2 e$

To a mixture of sodium $p$-toluenesulfinate $(1.140 \mathrm{~g}, 6.4 \mathrm{mmol})$ and bis $(p$-fluorophenyl) disulfide $(0.508 \mathrm{~g}, 2.0 \mathrm{mmol})$ in $\mathrm{CH}_{2} \mathrm{Cl}_{2}(10 \mathrm{~mL})$ was added $\mathrm{I}_{2}(1.015 \mathrm{~g}, 4.0 \mathrm{mmol})$ with stirring. The mixture was stirred until the disulfide was consumed, then $\mathrm{CH}_{2} \mathrm{Cl}_{2}(50 \mathrm{~mL})$ was added followed by aq. $\mathrm{Na}_{2} \mathrm{SO}_{3}(1 \mathrm{M})$ with stirring until the $\mathrm{I}_{2}$ color disappeared. The organic layer was washed with $\mathrm{H}_{2} \mathrm{O}$ $(50 \mathrm{~mL} \times 2)$ and dried over $\mathrm{Na}_{2} \mathrm{SO}_{4}$. then the solvent was removed under reduced pressure and the residue was purified by column chromatography on silica gel (1/10 ethyl acetate/hexanes) to give S-p-fluorophenyl $p$-toluenethiosulfonate $\mathbf{2 e}(1.049 \mathrm{~g}, 93 \%)$ as a white solid. $\mathbf{2 b - d , ~ 2 f - j}$ and $\mathbf{2 n}$ were prepared following this procedure.

A typical procedure for the synthesis of thiosulfonates from alkyl halides and sodium thiosulfonate 2 .

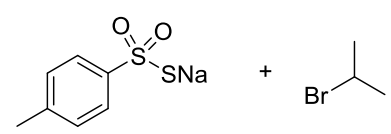

(2.0 equiv)

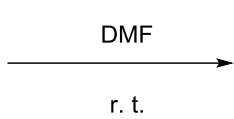

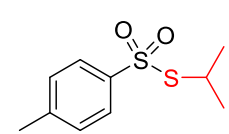

21

To a solution of sodium $p$-toluenethiosufonate $(0.631 \mathrm{~g}, 3.0 \mathrm{mmol})$ in DMF $(5 \mathrm{~mL})$ was added 2-bromopropane ( $0.56 \mathrm{~mL}, 6.0 \mathrm{mmol})$. After being stirred at room temperature for $16 \mathrm{~h}$, water (30 $\mathrm{mL})$ was added, and the mixture was extracted with EtOAc $(30 \mathrm{~mL} \times 3)$. The combined organic phase was dried over $\mathrm{Na}_{2} \mathrm{SO}_{4}$. The solvent was removed under reduced pressure and the residue was purified by column chromatography on silica gel (1/10 ethyl acetate/hexanes) to afford $\mathbf{2 l}(0.466 \mathrm{~g}$, quant.) as colorless oil. $\mathbf{2 k}$ and $\mathbf{2 m}$ were prepared following this procedure.

All thiosulfonates except $\mathbf{2 g}$ and $\mathbf{2 d}$ are known compounds and identical in the spectrum data reported by literatures.

\section{S-4-tert-butylphenyl $p$-toluenethiosulfonate (2g)}




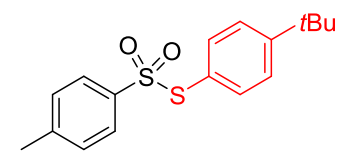

2g: $98 \%$ yield. White solid. It was purified with flash column chromatography on silica gel with ethyl acetate/hexanes (1/10) as eluent. ${ }^{1} \mathbf{H}$ NMR (400 MHz, $\left.\mathrm{CDCl}_{3}\right) \delta 7.47-7.41(\mathrm{~m}, 2 \mathrm{H}), 7.38-$ $7.32(\mathrm{~m}, 2 \mathrm{H}), 7.31-7.27(\mathrm{~m}, 2 \mathrm{H}), 7.23-7.17(\mathrm{~m}, 2 \mathrm{H}), 2.42(\mathrm{~s}, 3 \mathrm{H}), 1.31$ (s, 9H). ${ }^{13} \mathbf{C}$ NMR (100 $\left.\mathrm{MHz}, \mathrm{CDCl}_{3}\right) \delta 155.1,144.5,140.4,136.3,129.3,127.6,126.5,124.5,34.9,31.1,21.6$. HRMS (ESI) Calcd for $\mathrm{C}_{17} \mathrm{H}_{20} \mathrm{O}_{2} \mathrm{~S}_{2} \mathrm{Na}[\mathrm{M}+\mathrm{Na}]^{+}$343.0802, found 343.0796.

\section{S-4-tolyl 2-mesitylenethiosulfonate (2d)}

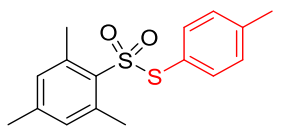

2d: $50 \%$ yield. White solid. It was purified with flash column chromatography on silica gel with ethyl acetate/hexanes (1/10) as eluent. ${ }^{1} \mathbf{H}$ NMR $\left(400 \mathrm{MHz}, \mathrm{CDCl}_{3}\right) \delta 7.20(\mathrm{~d}, J=8.4 \mathrm{~Hz}, 2 \mathrm{H}), 7.11$ $(\mathrm{d}, J=8.0 \mathrm{~Hz}, 2 \mathrm{H}), 6.87$ (s, 2H), 2.37 (s, 3H), $2.33(\mathrm{~s}, 6 \mathrm{H}), 2.30(\mathrm{~s}, 3 \mathrm{H}) .{ }^{13} \mathrm{C}$ NMR $(100 \mathrm{MHz}$, $\left.\mathrm{CDCl}_{3}\right) \delta 143.5,141.9,140.1,137.1,136.9,131.7,130.0,124.5,22.6,21.4,21.1$. HRMS (ESI) Calcd for $\mathrm{C}_{16} \mathrm{H}_{18} \mathrm{O}_{2} \mathrm{~S}_{2} \mathrm{Na}[\mathrm{M}+\mathrm{Na}]^{+}$329.0646, found 329.0652.

\section{General Procedure for Palladium/NBE-Catalyzed ortho Thiolation.}
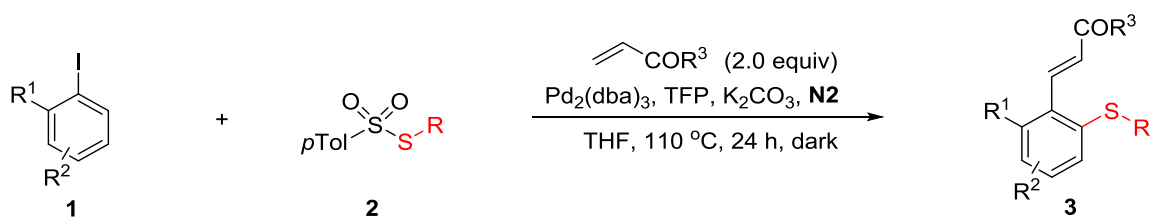

To a flame-dried sealed tube were added aryl iodide 1 ( $0.20 \mathrm{mmol}, 1.0$ equiv), thiosulfonate 2 (0.30 mmol, 1.5 equiv), acrylate ( $0.40 \mathrm{mmol}, 2.0$ equiv), $\mathrm{Pd}_{2}(\mathrm{dba})_{3}(9.2 \mathrm{mg}, 5 \mathrm{~mol} \%)$, TFP (10.2 mg, $22 \mathrm{~mol} \%$ ), bicyclo[2.2.1]hept-5-ene-2-carbaldehyde (N2) $(71 \mu \mathrm{L}, 0.60 \mathrm{mmol}, 3.0$ equiv), $\mathrm{K}_{2} \mathrm{CO}_{3}(82.9 \mathrm{mg}, 0.60 \mathrm{mmol}, 3.0$ equiv) and THF $(2.0 \mathrm{~mL})$. The mixture was allowed to stir at $110^{\circ} \mathrm{C}$ for $24 \mathrm{~h}$. After cooling down to room temperature, the mixture was filtered through a plug of silica gel eluting with ethyl acetate. The filtrate was concentrated and the residue was purified by column chromatography on silica gel to give the desired product 3. It's noted that light irradiation should be avoided as possible as it could during the reaction/purification and the product should be 
stored in darkness.

Synthesis of compound 3a

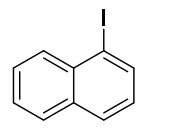

$1 \mathrm{a}$

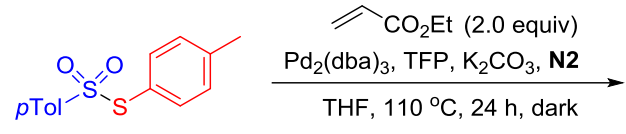

2a

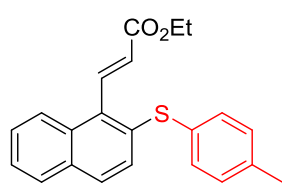

3a

The reaction of 1-iodonaphthalene $\mathbf{1 a}(50.8 \mathrm{mg}, 0.20 \mathrm{mmol}, 1.0$ equiv), $\mathbf{2 a}(83.5 \mathrm{mg}, 0.30 \mathrm{mmol}$, 1.5 equiv), ethyl acrylate ( $44 \mu \mathrm{L}, 0.4 \mathrm{mmol}, 2.0$ equiv), $\operatorname{Pd}_{2}(\mathrm{dba})_{3}(9.2 \mathrm{mg}, 5 \mathrm{~mol} \%)$, TFP (10.2 mg, $22 \mathrm{~mol} \%), \mathbf{N 2}\left(71 \mu \mathrm{L}, 0.60 \mathrm{mmol}, 3.0\right.$ equiv), $\mathrm{K}_{2} \mathrm{CO}_{3}(82.9 \mathrm{mg}, 0.60 \mathrm{mmol}, 3.0$ equiv) in THF (2.0 $\mathrm{mL}$ ) delivered 3a (59.0 mg, 84\%) (1/200 ethyl acetate/hexanes), ${ }^{1} \mathbf{H}$ NMR $\left(400 \mathrm{MHz}, \mathrm{CDCl}_{3}\right) \delta$ $8.27(\mathrm{~d}, J=16.4 \mathrm{~Hz}, 1 \mathrm{H}), 8.16-8.07(\mathrm{~m}, 1 \mathrm{H}), 7.83-7.73(\mathrm{~m}, 1 \mathrm{H}), 7.65(\mathrm{~d}, J=8.8 \mathrm{~Hz}, 1 \mathrm{H}), 7.56$ $-7.43(\mathrm{~m}, 2 \mathrm{H}), 7.36-7.27(\mathrm{~m}, 2 \mathrm{H}), 7.21(\mathrm{~d}, J=8.8 \mathrm{~Hz}, 1 \mathrm{H}), 7.18-7.10(\mathrm{~m}, 2 \mathrm{H}), 6.33(\mathrm{~d}, J=16.0$ $\mathrm{Hz}, 1 \mathrm{H}), 4.33$ (q, $J=7.2 \mathrm{~Hz}, 2 \mathrm{H}), 2.35(\mathrm{~s}, 3 \mathrm{H}), 1.38(\mathrm{t}, J=7.2 \mathrm{~Hz}, 3 \mathrm{H}) .{ }^{13} \mathbf{C ~ N M R}\left(100 \mathrm{MHz}, \mathrm{CDCl}_{3}\right)$ $\delta 166.4,140.9,138.0,135.0,132.7,132.2,131.4,131.0,130.1,129.1,128.4,127.5,127.2,126.4$, 125.9, 124.8, 60.7, 21.2, 14.3. HRMS (ESI) Calcd for $\mathrm{C}_{22} \mathrm{H}_{21} \mathrm{O}_{2} \mathrm{~S}[\mathrm{M}+\mathrm{H}]^{+}$349.1262, found 349.1254 .

Synthesis of compound $\mathbf{3 b}$

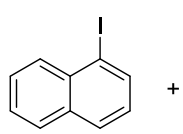

$1 \mathrm{a}$

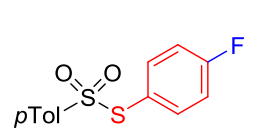

$2 e$

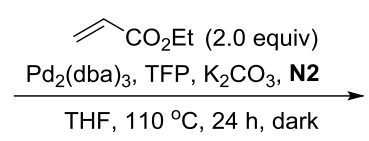

THF, $110^{\circ} \mathrm{C}, 24 \mathrm{~h}$, dark

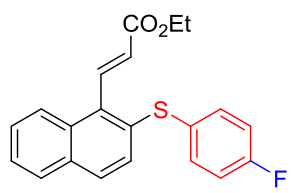

$3 \mathbf{b}$

The reaction of 1-iodonaphthalene 1a $(50.8 \mathrm{mg}, 0.20 \mathrm{mmol}, 1.0$ equiv), $2 \mathbf{e}(84.7 \mathrm{mg}, 0.30 \mathrm{mmol}$, 1.5 equiv), ethyl acrylate ( $44 \mu \mathrm{L}, 0.40 \mathrm{mmol}, 2.0$ equiv), $\mathrm{Pd}_{2}(\mathrm{dba})_{3}(9.2 \mathrm{mg}, 5 \mathrm{~mol} \%)$, TFP (10.2 mg, $22 \mathrm{~mol} \%), \mathbf{N} 2$ (71 $\mu \mathrm{L}, 0.60 \mathrm{mmol}, 3.0$ equiv), $\mathrm{K}_{2} \mathrm{CO}_{3}(82.9 \mathrm{mg}, 0.60 \mathrm{mmol}, 3.0$ equiv) in THF (2.0 mL) delivered $\mathbf{3 b}$ (41.3 mg, 58\%) (1/200 ethyl acetate/hexanes). ${ }^{1} \mathbf{H}$ NMR (400 MHz, $\mathrm{CDCl}_{3}$ ) $\delta 8.25(\mathrm{~d}, J=16.0 \mathrm{~Hz}, 1 \mathrm{H}), 8.16-8.08(\mathrm{~m}, 1 \mathrm{H}), 7.82-7.77(\mathrm{~m}, 1 \mathrm{H}), 7.68(\mathrm{~d}, J=9.2 \mathrm{~Hz}, 1 \mathrm{H}), 7.56$ $-7.45(\mathrm{~m}, 2 \mathrm{H}), 7.40-7.33(\mathrm{~m}, 2 \mathrm{H}), 7.22(\mathrm{~d}, J=8.8 \mathrm{~Hz}, 1 \mathrm{H}), 7.07-6.98(\mathrm{~m}, 2 \mathrm{H}), 6.31(\mathrm{~d}, J=16.4$ $\mathrm{Hz}, 1 \mathrm{H}), 4.33(\mathrm{q}, J=7.2 \mathrm{~Hz}, 2 \mathrm{H}), 1.38(\mathrm{t}, J=7.2 \mathrm{~Hz}, 3 \mathrm{H}) .{ }^{13} \mathbf{C} \mathbf{N M R}\left(100 \mathrm{MHz}, \mathrm{CDCl}_{3}\right) \delta 166.3$, $162.5(\mathrm{~d}, J=246.9 \mathrm{~Hz}), 140.8,134.4(\mathrm{~d}, J=8.1 \mathrm{~Hz}), 134.2,132.7,132.3,131.4,130.0(\mathrm{~d}, J=3.4$ 
Hz), 129.3, 128.4, 127.6, 127.3, 126.6, 126.2, 124.9, $116.5(\mathrm{~d}, J=21.8 \mathrm{~Hz}), 60.7,14.3 .{ }^{19} \mathbf{F}$ NMR (376 MHz, $\mathrm{CDCl}_{3}$ ) $\delta$-113.36. HRMS (ESI) Calcd for $\mathrm{C}_{21} \mathrm{H}_{17} \mathrm{O}_{2} \mathrm{FSNa}[\mathrm{M}+\mathrm{Na}]^{+}$375.0831, found 375.0828 .

Synthesis of compound $\mathbf{3 c}$

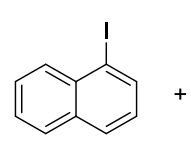

1a

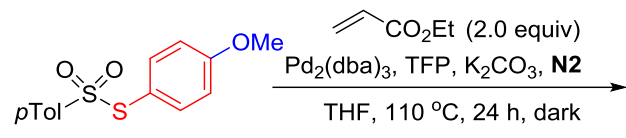

$2 \mathbf{f}$

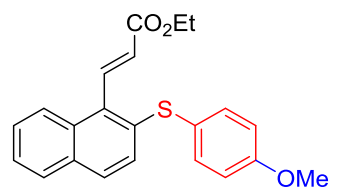

$3 c$

The reaction of 1-iodonaphthalene 1a $(50.8 \mathrm{mg}, 0.20 \mathrm{mmol}, 1.0$ equiv), $\mathbf{2 f}(88.3 \mathrm{mg}, 0.30 \mathrm{mmol}$, 1.5 equiv), ethyl acrylate ( $44 \mu \mathrm{L}, 0.40 \mathrm{mmol}, 2.0$ equiv), $\mathrm{Pd}_{2}(\mathrm{dba})_{3}(9.2 \mathrm{mg}, 5 \mathrm{~mol} \%)$, TFP (10.2 mg, $22 \mathrm{~mol} \%)$, $\mathbf{N} 2$ (71 $\mu \mathrm{L}, 0.60 \mathrm{mmol}, 3.0$ equiv), $\mathrm{K}_{2} \mathrm{CO}_{3}(82.9 \mathrm{mg}, 0.60 \mathrm{mmol}, 3.0$ equiv) in THF (2.0 mL) delivered 3c (59.2 mg, 81\%) (1/80 ethyl acetate/hexanes). ${ }^{1} \mathbf{H}$ NMR (400 MHz, $\left.\mathrm{CDCl}_{3}\right) \delta$ $8.27(\mathrm{~d}, J=16.0 \mathrm{~Hz}, 1 \mathrm{H}), 8.16-8.05(\mathrm{~m}, 1 \mathrm{H}), 7.85-7.72(\mathrm{~m}, 1 \mathrm{H}), 7.62(\mathrm{~d}, J=8.8 \mathrm{~Hz}, 1 \mathrm{H}), 7.56$ $-7.42(\mathrm{~m}, 2 \mathrm{H}), 7.42-7.37(\mathrm{~m}, 2 \mathrm{H}), 7.11(\mathrm{~d}, J=8.8 \mathrm{~Hz}, 1 \mathrm{H}), 6.94-6.87(\mathrm{~m}, 2 \mathrm{H}), 6.35(\mathrm{~d}, J=16.0$ $\mathrm{Hz}, 1 \mathrm{H}), 4.34$ (q, $J=7.2 \mathrm{~Hz}, 2 \mathrm{H}), 3.83(\mathrm{~s}, 3 \mathrm{H}), 1.39$ (t, $J=7.2 \mathrm{~Hz}, 3 \mathrm{H}) .{ }^{13} \mathbf{C} \mathbf{N M R}\left(100 \mathrm{MHz}, \mathrm{CDCl}_{3}\right)$ $\delta$ 166.4, 160.0, 140.7, 136.3, 135.5, 131.8, 131.4, 130.8, 129.1, 128.4, 127.2, 126.4, 126.3, 125.7, 124.6, 124.3, 115.1, 60.7, 55.4, 14.3. HRMS (ESI) Calcd for $\mathrm{C}_{22} \mathrm{H}_{20} \mathrm{O}_{3} \mathrm{SNa}[\mathrm{M}+\mathrm{Na}]^{+}$387.1031, found 387.1023 .

Synthesis of compound $\mathbf{3 d}$

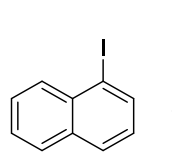

$1 \mathrm{a}$

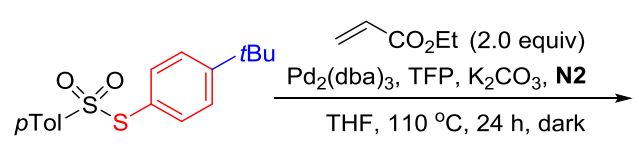

$2 \mathrm{~g}$

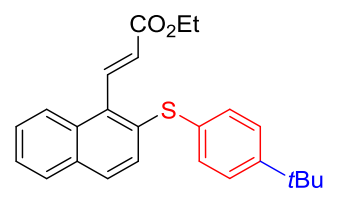

$3 d$

The reaction of 1-iodonaphthalene $\mathbf{1 a}(50.8 \mathrm{mg}, 0.20 \mathrm{mmol}, 1.0$ equiv), $\mathbf{2 g}$ (96.1 mg, $0.30 \mathrm{mmol}$, 1.5 equiv), ethyl acrylate ( $44 \mu \mathrm{L}, 0.40 \mathrm{mmol}, 2.0$ equiv), $\mathrm{Pd}_{2}(\mathrm{dba})_{3}(9.2 \mathrm{mg}, 5 \mathrm{~mol} \%)$, TFP (10.2 $\mathrm{mg}, 22 \mathrm{~mol} \%), \mathbf{N} 2\left(71 \mu \mathrm{L}, 0.60 \mathrm{mmol}, 3.0\right.$ equiv), $\mathrm{K}_{2} \mathrm{CO}_{3}(82.9 \mathrm{mg}, 0.60 \mathrm{mmol}, 3.0$ equiv) in THF (2.0 mL) delivered 3d (55.2 mg, 71\%) (1/200 ethyl acetate/hexanes). ${ }^{1} \mathbf{H}$ NMR (400 MHz, $\left.\mathrm{CDCl}_{3}\right)$ $\delta 8.28(\mathrm{~d}, J=16.0 \mathrm{~Hz}, 1 \mathrm{H}), 8.13(\mathrm{~d}, J=8.4 \mathrm{~Hz}, 1 \mathrm{H}), 7.83-7.75(\mathrm{~m}, 1 \mathrm{H}), 7.67(\mathrm{~d}, J=8.8 \mathrm{~Hz}, 1 \mathrm{H})$, $7.56-7.43(\mathrm{~m}, 2 \mathrm{H}), 7.38-7.28(\mathrm{~m}, 4 \mathrm{H}), 7.27(\mathrm{~d}, J=8.8 \mathrm{~Hz}, 1 \mathrm{H}), 6.33(\mathrm{~d}, J=16.4 \mathrm{~Hz}, 1 \mathrm{H}), 4.32$ 
$(\mathrm{q}, J=7.2 \mathrm{~Hz}, 2 \mathrm{H}), 1.37(\mathrm{t}, J=7.2 \mathrm{~Hz}, 3 \mathrm{H}), 1.31(\mathrm{~s}, 9 \mathrm{H}) .{ }^{13} \mathrm{C} \mathbf{N M R}\left(100 \mathrm{MHz}, \mathrm{CDCl}_{3}\right) \delta 166.4$, 151.0, 140.9, 134.7, 132.5, 132.3, 132.0, 131.5, 131.2, 129.2, 128.4, 127.9, 127.2, 126.40, 126.38, 126.0, 124.8, 60.7, 34.6, 31.2, 14.3. HRMS (ESI) Calcd for $\mathrm{C}_{25} \mathrm{H}_{26} \mathrm{O}_{2} \mathrm{SNa}[\mathrm{M}+\mathrm{Na}]^{+} 413.1551$, found 413.1544.

Synthesis of compound $\mathbf{3 e}$

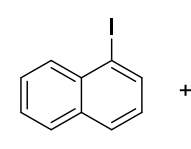

$1 a$

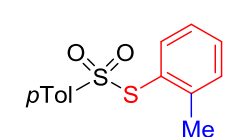

2h

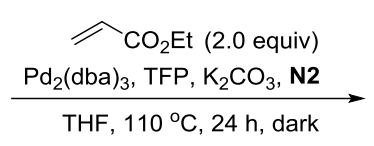

THF, $110^{\circ} \mathrm{C}, 24 \mathrm{~h}$, dark

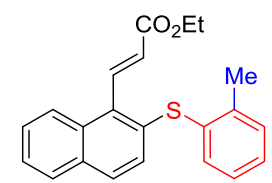

$3 e$

The reaction of 1-iodonaphthalene $\mathbf{1 a}(50.8 \mathrm{mg}, 0.20 \mathrm{mmol}, 1.0$ equiv), $\mathbf{2 h}(83.5 \mathrm{mg}, 0.30 \mathrm{mmol}$, 1.5 equiv), ethyl acrylate ( $44 \mu \mathrm{L}, 0.40 \mathrm{mmol}, 2.0$ equiv), $\mathrm{Pd}_{2}(\mathrm{dba})_{3}(9.2 \mathrm{mg}, 5 \mathrm{~mol} \%)$, TFP (10.2 mg, $22 \mathrm{~mol} \%), \mathbf{N 2}\left(71 \mu \mathrm{L}, 0.60 \mathrm{mmol}, 3.0\right.$ equiv), $\mathrm{K}_{2} \mathrm{CO}_{3}(82.9 \mathrm{mg}, 0.60 \mathrm{mmol}, 3.0$ equiv) in THF (2.0 mL) delivered 3e (68.9 mg, 98\%) (1/200 ethyl acetate/hexanes). ${ }^{1} \mathbf{H}$ NMR (400 MHz, $\left.\mathrm{CDCl}_{3}\right)$ $\delta 8.28(\mathrm{~d}, J=16.4 \mathrm{~Hz}, 1 \mathrm{H}), 8.16-8.08(\mathrm{~m}, 1 \mathrm{H}), 7.82-7.72(\mathrm{~m}, 1 \mathrm{H}), 7.63(\mathrm{~d}, J=8.8 \mathrm{~Hz}, 1 \mathrm{H}), 7.56$ $-7.41(\mathrm{~m}, 2 \mathrm{H}), 7.35-7.30(\mathrm{~m}, 1 \mathrm{H}), 7.28-7.22(\mathrm{~m}, 2 \mathrm{H}), 7.19-7.12(\mathrm{~m}, 1 \mathrm{H}), 7.08(\mathrm{~d}, J=8.8 \mathrm{~Hz}$, $1 \mathrm{H}), 6.36(\mathrm{~d}, J=16.4 \mathrm{~Hz}, 1 \mathrm{H}), 4.33(\mathrm{q}, J=7.2 \mathrm{~Hz}, 2 \mathrm{H}), 2.35(\mathrm{~s}, 3 \mathrm{H}), 1.38(\mathrm{t}, J=7.2 \mathrm{~Hz}, 3 \mathrm{H}) .{ }^{13} \mathrm{C}$ NMR $\left(100 \mathrm{MHz}, \mathrm{CDCl}_{3}\right) \delta 166.4,140.7,140.5,134.4,133.8,133.4,132.1,132.0,131.5,130.8$, 129.2, 128.43, 128.36, 127.2, 126.9, 126.8, 126.4, 125.9, 124.7, 60.7, 20.7, 14.3. HRMS (ESI) Calcd for $\mathrm{C}_{22} \mathrm{H}_{21} \mathrm{O}_{2} \mathrm{~S}[\mathrm{M}+\mathrm{H}]^{+}$349.1262, found 349.1251.

Synthesis of compound $\mathbf{3 f}$

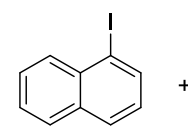

$1 a$

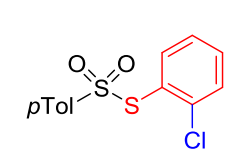

$2 \mathbf{i}$

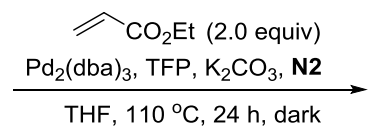

THF, $110^{\circ} \mathrm{C}, 24 \mathrm{~h}$, dark

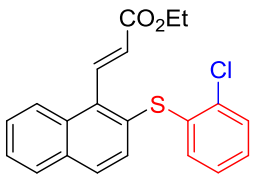

$3 f$

The reaction of 1-iodonaphthalene $\mathbf{1 a}(50.8 \mathrm{mg}, 0.20 \mathrm{mmol}, 1.0$ equiv), $\mathbf{2 i}$ ( $89.6 \mathrm{mg}, 0.30 \mathrm{mmol}$, 1.5 equiv), ethyl acrylate ( $44 \mu \mathrm{L}, 0.40 \mathrm{mmol}, 2.0$ equiv), $\mathrm{Pd}_{2}(\mathrm{dba})_{3}(9.2 \mathrm{mg}, 5 \mathrm{~mol} \%)$, TFP (10.2 $\mathrm{mg}, 22 \mathrm{~mol} \%), \mathbf{N 2}\left(71 \mu \mathrm{L}, 0.60 \mathrm{mmol}, 3.0\right.$ equiv), $\mathrm{K}_{2} \mathrm{CO}_{3}(82.9 \mathrm{mg}, 0.60 \mathrm{mmol}, 3.0$ equiv) in THF (2.0 mL) delivered 3f (41.6 mg, 56\%) (1/200 ethyl acetate/hexanes). ${ }^{1} \mathbf{H}$ NMR (400 MHz, $\left.\mathrm{CDCl}_{3}\right)$ $\delta 8.24(\mathrm{~d}, J=16.0 \mathrm{~Hz}, 1 \mathrm{H}), 8.18-8.09(\mathrm{~m}, 1 \mathrm{H}), 7.89-7.81(\mathrm{~m}, 1 \mathrm{H}), 7.75(\mathrm{~d}, J=8.8 \mathrm{~Hz}, 1 \mathrm{H}), 7.59$ 
$-7.50(\mathrm{~m}, 2 \mathrm{H}), 7.41(\mathrm{dd}, J=7.8,1.6 \mathrm{~Hz}, 1 \mathrm{H}), 7.34(\mathrm{~d}, J=8.4 \mathrm{~Hz}, 1 \mathrm{H}), 7.17(\mathrm{td}, J=7.8,2.0 \mathrm{~Hz}$, 1H), $7.11(\mathrm{td}, J=7.8,1.6 \mathrm{~Hz}, 1 \mathrm{H}), 7.05(\mathrm{dd}, J=7.8,1.6 \mathrm{~Hz}, 1 \mathrm{H}), 6.29(\mathrm{~d}, J=16.0 \mathrm{~Hz}, 1 \mathrm{H}), 4.31$ $(\mathrm{q}, J=7.2 \mathrm{~Hz}, 2 \mathrm{H}), 1.36(\mathrm{t}, J=7.2 \mathrm{~Hz}, 3 \mathrm{H}) .{ }^{13} \mathbf{C}$ NMR $\left(100 \mathrm{MHz}, \mathrm{CDCl}_{3}\right) \delta 166.2,140.8,135.21$, 135.17, 134.6, 133.0, 131.8, 131.6, 131.2, 130.0, 129.5, 129.4, 128.4, 128.0, 127.3, 126.7, 126.6, 125.2, 60.7, 14.3. HRMS (ESI) Calcd for $\mathrm{C}_{21} \mathrm{H}_{18} \mathrm{ClO}_{2} \mathrm{~S}[\mathrm{M}+\mathrm{H}]^{+}$369.0716, found 369.0711.

\section{Preparation of 3f at $1.0 \mathrm{mmol}$ scale}

The reaction of 1-iodonaphthalene 1a (254 mg, $1.0 \mathrm{mmol}, 1.0$ equiv), $2 \mathbf{i}$ (448 mg, $1.5 \mathrm{mmol}, 1.5$ equiv), ethyl acrylate ( $0.22 \mathrm{~mL}, 2.0 \mathrm{mmol}, 2.0$ equiv), $\mathrm{Pd}_{2}(\mathrm{dba})_{3}(46 \mathrm{mg}, 5 \mathrm{~mol} \%)$, TFP (51 mg, 22 mol\%), $\mathbf{N 2}$ (0.35 mL, $3.0 \mathrm{mmol}, 3.0$ equiv), $\mathrm{K}_{2} \mathrm{CO}_{3}(415 \mathrm{mg}, 3.0 \mathrm{mmol}, 3.0$ equiv) in THF (10.0 $\mathrm{mL}$ ) delivered $\mathbf{3 f}$ (164 mg, 44\%) (1/200 ethyl acetate/hexanes). The analytic data was identical with the compound obtained in $0.2 \mathrm{mmol}$ scale.

Synthesis of compound $\mathbf{3 g}$

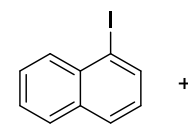

$1 \mathrm{a}$

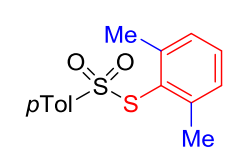

2j

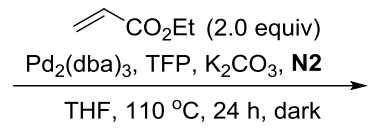

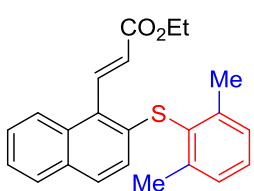

$3 \mathrm{~g}$

The reaction of 1-iodonaphthalene $\mathbf{1 a}(50.8 \mathrm{mg}, 0.20 \mathrm{mmol}, 1.0$ equiv), $\mathbf{2 j}$ ( $87.7 \mathrm{mg}, 0.30 \mathrm{mmol}$, 1.5 equiv), ethyl acrylate ( $44 \mu \mathrm{L}, 0.40 \mathrm{mmol}, 2.0$ equiv), $\mathrm{Pd}_{2}(\mathrm{dba})_{3}(9.2 \mathrm{mg}, 5 \mathrm{~mol} \%)$, TFP (10.2 mg, $22 \mathrm{~mol} \%)$, $\mathbf{N 2}$ (71 $\mu \mathrm{L}, 0.60 \mathrm{mmol}, 3.0$ equiv), $\mathrm{K}_{2} \mathrm{CO}_{3}(82.9 \mathrm{mg}, 0.60 \mathrm{mmol}, 3.0$ equiv) in THF (2.0 mL) delivered 3g (57.4 mg, 79\%) (1/200 ethyl acetate/hexanes). ${ }^{1} \mathbf{H}$ NMR (400 MHz, $\mathrm{CDCl}_{3}$ ) $\delta 8.31(\mathrm{~d}, J=16.4 \mathrm{~Hz}, 1 \mathrm{H}), 8.16-8.07(\mathrm{~m}, 1 \mathrm{H}), 7.79-7.71(\mathrm{~m}, 1 \mathrm{H}), 7.57-7.48(\mathrm{~m}, 2 \mathrm{H}), 7.46-$ $7.37(\mathrm{~m}, 1 \mathrm{H}), 7.29-7.23(\mathrm{~m}, 1 \mathrm{H}), 7.22-7.17(\mathrm{~m}, 2 \mathrm{H}), 6.70(\mathrm{~d}, J=8.8 \mathrm{~Hz}, 1 \mathrm{H}), 6.46(\mathrm{~d}, J=16.4$ $\mathrm{Hz}, 1 \mathrm{H}), 4.36(\mathrm{q}, J=7.2 \mathrm{~Hz}, 2 \mathrm{H}), 2.39(\mathrm{~s}, 6 \mathrm{H}), 1.41(\mathrm{t}, J=7.2 \mathrm{~Hz}, 3 \mathrm{H}) .{ }^{13} \mathbf{C ~ N M R}\left(100 \mathrm{MHz}, \mathrm{CDCl}_{3}\right)$ $\delta 166.6,143.8,140.4,136.1,131.6,131.4,131.0,129.4,129.2,128.6,128.4,127.2,126.3,125.3$, 124.2, 123.5, 60.7, 21.9, 14.4. HRMS (ESI) Calcd for $\mathrm{C}_{23} \mathrm{H}_{23} \mathrm{O}_{2} \mathrm{~S}[\mathrm{M}+\mathrm{H}]^{+} 363.1419$, found 363.1422 .

Synthesis of compound $\mathbf{3 h}$ 


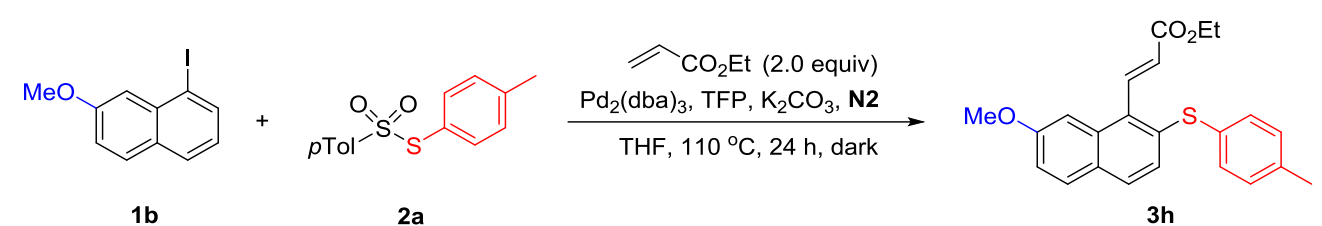

The reaction of 1-iodo-7-methoxynaphthalene $\mathbf{1 b}(56.8 \mathrm{mg}, 0.20 \mathrm{mmol}, 1.0$ equiv), $\mathbf{2 a}(83.5 \mathrm{mg}$, $0.30 \mathrm{mmol}, 1.5$ equiv), ethyl acrylate ( $44 \mu \mathrm{L}, 0.40 \mathrm{mmol}, 2.0$ equiv), $\operatorname{Pd}_{2}(\mathrm{dba})_{3}(9.2 \mathrm{mg}, 5 \mathrm{~mol} \%)$, TFP (10.2 mg, $22 \mathrm{~mol} \%)$, N2 (71 $\mu \mathrm{L}, 0.60 \mathrm{mmol}, 3.0$ equiv), $\mathrm{K}_{2} \mathrm{CO}_{3}(82.9 \mathrm{mg}, 0.60 \mathrm{mmol}, 3.0$ equiv) in THF (2.0 mL) delivered $\mathbf{3 h}(55.6 \mathrm{mg}, 73 \%)$ (1/50 ethyl acetate/hexanes). ${ }^{\mathbf{1}} \mathbf{H}$ NMR (400 MHz, $\left.\mathrm{CDCl}_{3}\right) \delta 8.24(\mathrm{~d}, J=16.0 \mathrm{~Hz}, 1 \mathrm{H}), 7.68(\mathrm{~d}, J=9.2 \mathrm{~Hz}, 1 \mathrm{H}), 7.57(\mathrm{~d}, J=8.4 \mathrm{~Hz}, 1 \mathrm{H}), 7.40(\mathrm{~d}, J=$ $2.4 \mathrm{~Hz}, 1 \mathrm{H}), 7.33-7.27(\mathrm{~m}, 2 \mathrm{H}), 7.18-7.11(\mathrm{~m}, 3 \mathrm{H}), 7.06(\mathrm{~d}, J=8.8 \mathrm{~Hz}, 1 \mathrm{H}), 6.37(\mathrm{~d}, J=16.4$ $\mathrm{Hz}, 1 \mathrm{H}), 4.32(\mathrm{q}, J=7.2 \mathrm{~Hz}, 2 \mathrm{H}), 3.91(\mathrm{~s}, 3 \mathrm{H}), 2.35(\mathrm{~s}, 3 \mathrm{H}), 1.37(\mathrm{t}, J=7.2 \mathrm{~Hz}, 3 \mathrm{H}) .{ }^{13} \mathbf{C}$ NMR $\left(100 \mathrm{MHz}, \mathrm{CDCl}_{3}\right) \delta 166.5,158.7,141.3,138.0,135.8,132.78,132.77,131.0,130.7,130.1,129.9$, 128.9, 127.5, 126.0, 125.2, 118.1, 103.7, 60.7, 55.4, 21.2, 14.3. HRMS (ESI) Calcd for $\mathrm{C}_{23} \mathrm{H}_{22} \mathrm{O}_{3} \mathrm{SNa}[\mathrm{M}+\mathrm{Na}]^{+}$401.1187, found 401.1190.

Synthesis of compound $\mathbf{3 i}$
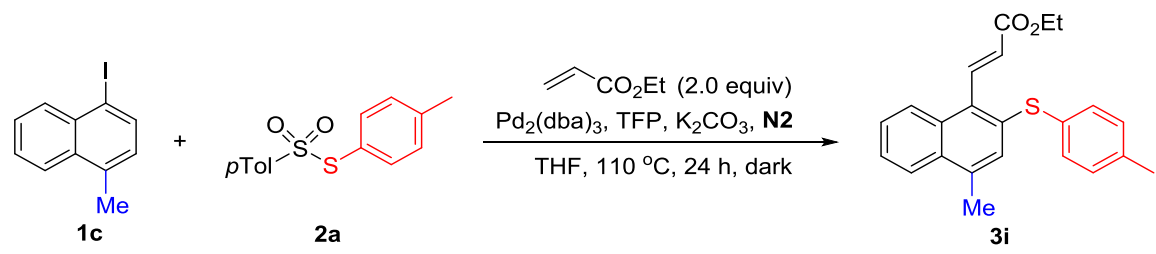

The reaction of 1-iodo-4-methylnaphthalene $\mathbf{1 c}(56.8 \mathrm{mg}, 0.20 \mathrm{mmol}, 1.0$ equiv), $\mathbf{2 a}(83.5 \mathrm{mg}$, $0.30 \mathrm{mmol}, 1.5$ equiv), ethyl acrylate (44 $\mu \mathrm{L}, 0.40 \mathrm{mmol}, 2.0$ equiv), $\operatorname{Pd}_{2}(\mathrm{dba})_{3}(9.2 \mathrm{mg}, 5 \mathrm{~mol} \%)$, TFP (10.2 mg, $22 \mathrm{~mol} \%)$, $\mathbf{N 2}\left(71 \mu \mathrm{L}, 0.60 \mathrm{mmol}, 3.0\right.$ equiv), $\mathrm{K}_{2} \mathrm{CO}_{3}(82.9 \mathrm{mg}, 0.60 \mathrm{mmol}, 3.0$ equiv) in THF (2.0 mL) delivered 3i $(57.4 \mathrm{mg}, 79 \%)\left(1 / 200\right.$ ethyl acetate/hexanes). ${ }^{1} \mathbf{H}$ NMR (400 MHz, $\left.\mathrm{CDCl}_{3}\right) \delta 8.28(\mathrm{~d}, J=16.4 \mathrm{~Hz}, 1 \mathrm{H}), 8.19-8.10(\mathrm{~m}, 1 \mathrm{H}), 7.98-7.87(\mathrm{~m}, 1 \mathrm{H}), 7.57-7.45(\mathrm{~m}, 2 \mathrm{H})$, $7.29-7.20(\mathrm{~m}, 2 \mathrm{H}), 7.17-7.08(\mathrm{~m}, 3 \mathrm{H}), 6.29(\mathrm{~d}, J=16.0 \mathrm{~Hz}, 1 \mathrm{H}), 4.31(\mathrm{q}, J=7.2 \mathrm{~Hz}, 2 \mathrm{H}), 2.55$ (s, 3H), $2.34(\mathrm{~s}, 3 \mathrm{H}), 1.37(\mathrm{t}, J=7.2 \mathrm{~Hz}, 3 \mathrm{H}) .{ }^{13} \mathrm{C} \mathbf{N M R}\left(101 \mathrm{MHz}, \mathrm{CDCl}_{3}\right) \delta 166.5,141.2,137.6$, $135.8,134.0,132.1,131.64,131.59,131.4,131.1,130.1,128.5,126.8,126.1,125.9,125.5,124.5$, 60.6, 21.1, 19.5, 14.3. HRMS (ESI) Calcd for $\mathrm{C}_{23} \mathrm{H}_{23} \mathrm{O}_{2} \mathrm{~S}[\mathrm{M}+\mathrm{H}]^{+}$363.1419, found 363.1417.

Synthesis of compound 3j 


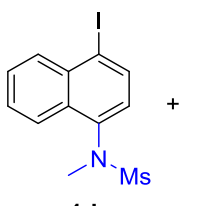

1d

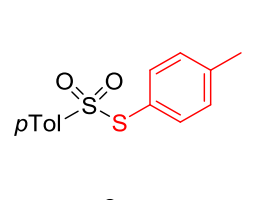

2a
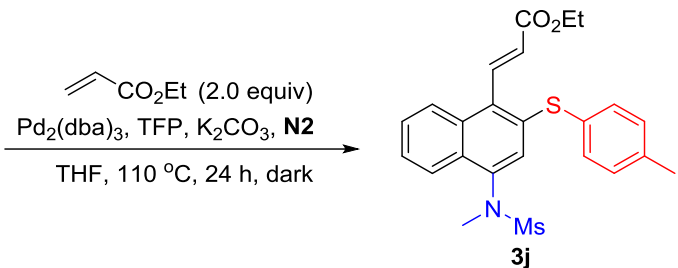

3j

The reaction of $\mathbf{1 d}(72.2 \mathrm{mg}, 0.20 \mathrm{mmol}, 1.0$ equiv), $\mathbf{2 a}(83.5 \mathrm{mg}, 0.30 \mathrm{mmol}, 1.5$ equiv), ethyl acrylate (44 $\mu \mathrm{L}, 0.40 \mathrm{mmol}, 2.0$ equiv), $\mathrm{Pd}_{2}(\mathrm{dba})_{3}(9.2 \mathrm{mg}, 5 \mathrm{~mol} \%)$, TFP (10.2 mg, $\left.22 \mathrm{~mol} \%\right), \mathbf{N 2}$ (71 $\mu \mathrm{L}, 0.60 \mathrm{mmol}, 3.0$ equiv), $\mathrm{K}_{2} \mathrm{CO}_{3}(82.9 \mathrm{mg}, 0.60 \mathrm{mmol}, 3.0$ equiv) in THF (2.0 mL) delivered 3j (60.8 mg, 67\%) (100/50 to 100/0 DCM/hexanes). ${ }^{1} \mathbf{H}$ NMR $\left(400 \mathrm{MHz}, \mathrm{CDCl}_{3}\right) \delta 8.20$ (d, $J=$ $16.0 \mathrm{~Hz}, 1 \mathrm{H}), 8.22-8.16(\mathrm{~m}, 1 \mathrm{H}), 8.14-8.07(\mathrm{~m}, 1 \mathrm{H}), 7.61-7.54(\mathrm{~m}, 2 \mathrm{H}), 7.34-7.29(\mathrm{~m}, 2 \mathrm{H})$, $7.20-7.15(\mathrm{~m}, 2 \mathrm{H}), 7.15(\mathrm{~s}, 1 \mathrm{H}), 6.33(\mathrm{~d}, J=16.0 \mathrm{~Hz}, 1 \mathrm{H}), 4.34(\mathrm{q}, J=7.2 \mathrm{~Hz}, 2 \mathrm{H}), 3.26(\mathrm{~s}, 3 \mathrm{H})$, $2.78(\mathrm{~s}, 3 \mathrm{H}), 2.36(\mathrm{~s}, 3 \mathrm{H}), 1.39(\mathrm{t}, J=7.2 \mathrm{~Hz}, 3 \mathrm{H}) .{ }^{13} \mathbf{C} \mathbf{N M R}\left(100 \mathrm{MHz}, \mathrm{CDCl}_{3}\right) \delta 166.1,140.1$, $138.8,138.6,134.8,133.0,132.9,132.8,130.53,130.52,130.3,128.0,127.4,127.1,126.3,125.2$, 124.2, 60.9, 39.4, 36.4, 21.2, 14.3. HRMS (ESI) Calcd for $\mathrm{C}_{24} \mathrm{H}_{25} \mathrm{NO}_{4} \mathrm{~S}_{2} \mathrm{Na}[\mathrm{M}+\mathrm{Na}]^{+} 478.1123$, found 478.1120

Synthesis of compound $\mathbf{3 k}$

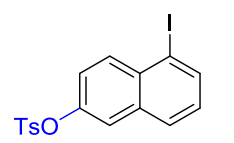

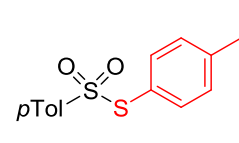

2a

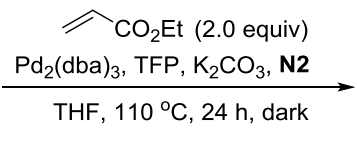

THF, $110^{\circ} \mathrm{C}, 24 \mathrm{~h}$, dark

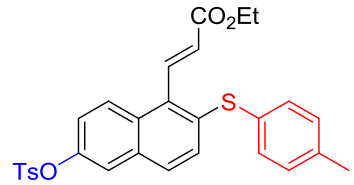

3k

The reaction of $1 \mathbf{e}(84.9 \mathrm{mg}, 0.20 \mathrm{mmol}, 1.0$ equiv), $2 \mathrm{a}(83.5 \mathrm{mg}, 0.30 \mathrm{mmol}, 1.5$ equiv), ethyl acrylate (44 $\mu \mathrm{L}, 0.40 \mathrm{mmol}, 2.0$ equiv), $\mathrm{Pd}_{2}(\mathrm{dba})_{3}(9.2 \mathrm{mg}, 5 \mathrm{~mol} \%)$, TFP (10.2 mg, $\left.22 \mathrm{~mol} \%\right), \mathbf{N 2}$ (71 $\mu \mathrm{L}, 0.60 \mathrm{mmol}, 3.0$ equiv), $\mathrm{K}_{2} \mathrm{CO}_{3}(82.9 \mathrm{mg}, 0.60 \mathrm{mmol}, 3.0$ equiv) in THF (2.0 mL) delivered 3k (70.3 mg, 67\%) (1/10 ethyl acetate/hexanes). ${ }^{1} \mathbf{H}$ NMR (400 MHz, $\left.\mathrm{CDCl}_{3}\right) \delta 8.18(\mathrm{~d}, J=16.0$ $\mathrm{Hz}, 1 \mathrm{H}), 7.99(\mathrm{~d}, J=9.6 \mathrm{~Hz}, 1 \mathrm{H}), 7.75-7.68(\mathrm{~m}, 2 \mathrm{H}), 7.54(\mathrm{~d}, J=8.8 \mathrm{~Hz}, 1 \mathrm{H}), 7.46(\mathrm{~d}, J=2.4 \mathrm{~Hz}$, 1H), $7.33-7.27(\mathrm{~m}, 4 \mathrm{H}), 7.21-7.13(\mathrm{~m}, 3 \mathrm{H}), 7.04(\mathrm{dd}, J=9.2,2.4 \mathrm{~Hz}, 1 \mathrm{H}), 6.28(\mathrm{~d}, J=16.4 \mathrm{~Hz}$, 1H), $4.31(\mathrm{q}, J=7.2 \mathrm{~Hz}, 2 \mathrm{H}), 2.45(\mathrm{~s}, 3 \mathrm{H}), 2.36(\mathrm{~s}, 3 \mathrm{H}), 1.37(\mathrm{t}, J=7.2 \mathrm{~Hz}, 3 \mathrm{H}) .{ }^{13} \mathbf{C}$ NMR $(100$ $\left.\mathrm{MHz}, \mathrm{CDCl}_{3}\right) \delta 166.2,147.1,145.5,140.3,138.5,136.3,133.2,132.3,131.6,130.3,130.2,129.9$, $129.8,128.8,128.5,128.1,126.9,126.7,122.1,120.5,60.8,21.7,21.2,14.3$. Calcd for $\mathrm{C}_{29} \mathrm{H}_{27} \mathrm{O}_{5} \mathrm{~S}_{2}$ $[\mathrm{M}+\mathrm{H}]^{+}$519.1300, found 519.1304. 
Synthesis of compound $\mathbf{3 l}$

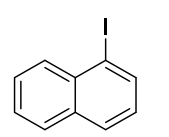

$1 \mathrm{a}$

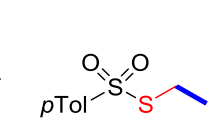

$\mathbf{2 k}$

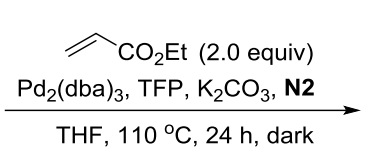

THF, $110^{\circ} \mathrm{C}, 24 \mathrm{~h}$, dark

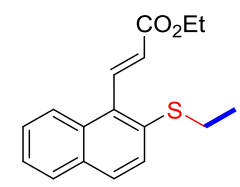

31

The reaction of $1 \mathbf{a}(50.8 \mathrm{mg}, 0.20 \mathrm{mmol}, 1.0$ equiv), $2 \mathbf{k}(64.9 \mathrm{mg}, 0.30 \mathrm{mmol}, 1.5$ equiv), ethyl acrylate (44 $\mu \mathrm{L}, 0.40 \mathrm{mmol}, 2.0$ equiv), $\mathrm{Pd}_{2}(\mathrm{dba})_{3}(9.2 \mathrm{mg}, 5 \mathrm{~mol} \%)$, TFP (10.2 mg, $\left.22 \mathrm{~mol} \%\right), \mathbf{N 2}$ (71 $\mu \mathrm{L}, 0.60 \mathrm{mmol}, 3.0$ equiv), $\mathrm{K}_{2} \mathrm{CO}_{3}(82.9 \mathrm{mg}, 0.60 \mathrm{mmol}, 3.0$ equiv) in THF (2.0 mL) delivered 31 (33.0 mg, 57\%) (1/100 ethyl acetate/hexanes). ${ }^{\mathbf{1}} \mathbf{H}$ NMR (400 MHz, $\left.\mathrm{CDCl}_{3}\right) \delta 8.24$ (d, $J=16.0$ $\mathrm{Hz}, 1 \mathrm{H}), 8.17-8.05(\mathrm{~m}, 1 \mathrm{H}), 7.84-7.79(\mathrm{~m}, 1 \mathrm{H}), 7.78(\mathrm{~d}, J=8.8 \mathrm{~Hz}, 1 \mathrm{H}), 7.55-7.42(\mathrm{~m}, 3 \mathrm{H})$, $6.33(\mathrm{~d}, J=16.4 \mathrm{~Hz}, 1 \mathrm{H}), 4.34(\mathrm{q}, J=7.2 \mathrm{~Hz}, 2 \mathrm{H}), 3.03(\mathrm{q}, J=7.2 \mathrm{~Hz}, 2 \mathrm{H}), 1.39(\mathrm{t}, J=7.2 \mathrm{~Hz}, 3 \mathrm{H})$, $1.32(\mathrm{t}, J=7.2 \mathrm{~Hz}, 3 \mathrm{H}) .{ }^{13} \mathbf{C}$ NMR $\left(100 \mathrm{MHz}, \mathrm{CDCl}_{3}\right) \delta 166.5,141.2,134.3,132.4,131.9,131.4$, 129.0, 128.4, 127.1, 126.0, 125.9, 125.7, 124.7, 60.7, 28.0, 14.5, 14.3. HRMS (ESI) Calcd for $\mathrm{C}_{17} \mathrm{H}_{18} \mathrm{O}_{2} \mathrm{SNa}[\mathrm{M}+\mathrm{Na}]^{+}$309.0925, found 309.0927.

Synthesis of compound $\mathbf{3 m}$

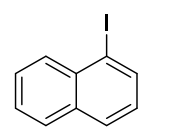

$1 \mathbf{a}$

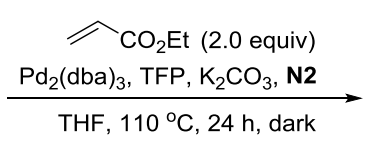

2l

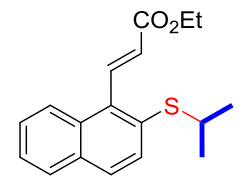

$3 m$

The reaction of 1a (50.8 mg, $0.20 \mathrm{mmol}, 1.0$ equiv), $2 \mathrm{l}(69.1 \mathrm{mg}, 0.30 \mathrm{mmol}, 1.5$ equiv), ethyl acrylate (44 $\mu \mathrm{L}, 0.40 \mathrm{mmol}, 2.0$ equiv), $\mathrm{Pd}_{2}(\mathrm{dba})_{3}(9.2 \mathrm{mg}, 5 \mathrm{~mol} \%)$, TFP (10.2 mg, $\left.22 \mathrm{~mol} \%\right), \mathbf{N 2}$ (71 $\mu \mathrm{L}, 0.60 \mathrm{mmol}, 3.0$ equiv), $\mathrm{K}_{2} \mathrm{CO}_{3}(82.9 \mathrm{mg}, 0.6 \mathrm{mmol}, 3.0$ equiv) in THF (2.0 mL) delivered 3m (41.3 mg, 68\%) (1/100 ethyl acetate/hexanes). ${ }^{1} \mathbf{H}$ NMR (400 MHz, $\left.\mathrm{CDCl}_{3}\right) \delta 8.28$ (d, $J=16.0$ Hz, 1H), $8.19-8.10(\mathrm{~m}, 1 \mathrm{H}), 7.86-7.79(\mathrm{~m}, 1 \mathrm{H}), 7.77$ (d, $J=8.8 \mathrm{~Hz}, 1 \mathrm{H}), 7.56$ (d, $J=8.8 \mathrm{~Hz}$, 1H), $7.53-7.44$ (m, 2H), 6.30 (d, $J=16.4 \mathrm{~Hz}, 1 \mathrm{H}), 4.34$ (q, $J=7.2 \mathrm{~Hz}, 2 \mathrm{H}), 3.52$ (hept, $J=6.8 \mathrm{~Hz}$, 1H), $1.39(\mathrm{t}, J=7.2 \mathrm{~Hz}, 3 \mathrm{H}), 1.32(\mathrm{~d}, J=6.4 \mathrm{~Hz}, 6 \mathrm{H}) .{ }^{13} \mathbf{C} \mathbf{~ N M R}\left(100 \mathrm{MHz}, \mathrm{CDCl}_{3}\right) \delta 166.6,141.7$, 134.1, 133.8, 132.3, 131.5, 128.8, 128.44, 128.36, 127.0, 125.9, 125.8, 125.0, 60.6, 38.6, 23.1, 14.3. HRMS (ESI) Calcd for $\mathrm{C}_{18} \mathrm{H}_{21} \mathrm{O}_{2} \mathrm{~S}[\mathrm{M}+\mathrm{H}]^{+}$301.1262, found 301.1272. 
Synthesis of compound $\mathbf{3 n}$

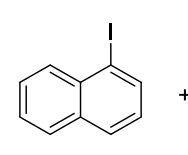

$1 \mathrm{a}$

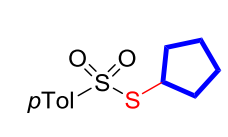

$2 m$

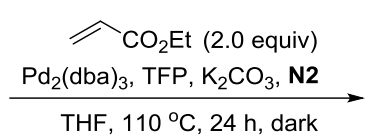

THF, $110^{\circ} \mathrm{C}, 24 \mathrm{~h}$, dark

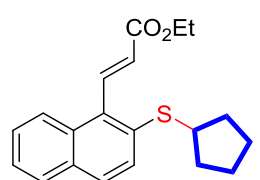

$3 n$

The reaction of $1 \mathbf{a}(50.8 \mathrm{mg}, 0.20 \mathrm{mmol}, 1.0$ equiv), $\mathbf{2 m}$ ( $76.9 \mathrm{mg}, 0.30 \mathrm{mmol}, 1.5$ equiv), ethyl acrylate (44 $\mu \mathrm{L}, 0.40 \mathrm{mmol}, 2.0$ equiv), $\mathrm{Pd}_{2}(\mathrm{dba})_{3}(9.2 \mathrm{mg}, 5 \mathrm{~mol} \%)$, TFP (10.2 mg, $\left.22 \mathrm{~mol} \%\right), \mathbf{N 2}$ (71 $\mu \mathrm{L}, 0.60 \mathrm{mmol}, 3.0$ equiv), $\mathrm{K}_{2} \mathrm{CO}_{3}(82.9 \mathrm{mg}, 0.60 \mathrm{mmol}, 3.0$ equiv) in THF (2.0 mL) delivered 3n (42.9 mg, 66\%) (1/150 ethyl acetate/hexanes). ${ }^{1} \mathbf{H}$ NMR (400 MHz, $\left.\mathrm{CDCl}_{3}\right) \delta 8.23(\mathrm{~d}, J=16.0$ $\mathrm{Hz}, 1 \mathrm{H}), 8.17-8.06(\mathrm{~m}, 1 \mathrm{H}), 7.84-7.78(\mathrm{~m}, 1 \mathrm{H}), 7.76(\mathrm{~d}, J=8.8 \mathrm{~Hz}, 1 \mathrm{H}), 7.56(\mathrm{~d}, J=8.4 \mathrm{~Hz}$, 1H), $7.53-7.40(\mathrm{~m}, 2 \mathrm{H}), 6.31(\mathrm{~d}, J=16.4 \mathrm{~Hz}, 1 \mathrm{H}), 4.33(\mathrm{q}, J=7.2 \mathrm{~Hz}, 2 \mathrm{H}), 3.85-3.66(\mathrm{~m}, 1 \mathrm{H})$, $2.15-1.99(\mathrm{~m}, 2 \mathrm{H}), 1.90-1.75(\mathrm{~m}, 2 \mathrm{H}), 1.71-1.60(\mathrm{~m}, 4 \mathrm{H}), 1.38(\mathrm{t}, J=7.2 \mathrm{~Hz}, 3 \mathrm{H}) .{ }^{13} \mathbf{C} \mathbf{N M R}$ $\left(100 \mathrm{MHz}, \mathrm{CDCl}_{3}\right) \delta 166.6,141.4,135.4,132.5,131.9,131.4,128.8,128.4,127.14,127.06,125.9$, 125.6, 124.7, 60.6, 46.3, 33.6, 24.8, 14.3. HRMS (ESI) Calcd for $\mathrm{C}_{20} \mathrm{H}_{23} \mathrm{O}_{2} \mathrm{~S}[\mathrm{M}+\mathrm{H}]^{+}$327.1419, found 327.1412 .

Synthesis of compound $\mathbf{3 o}$

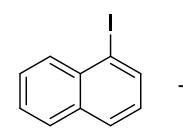

1a

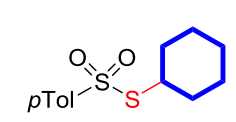

$2 n$

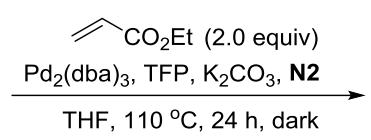

THF, $110^{\circ} \mathrm{C}, 24 \mathrm{~h}$, dark

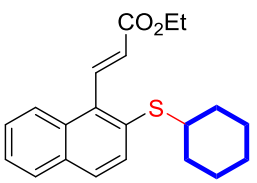

30

The reaction of $\mathbf{1 a}(50.8 \mathrm{mg}, 0.20 \mathrm{mmol}, 1.0$ equiv), $\mathbf{2 n}(81.1 \mathrm{mg}, 0.30 \mathrm{mmol}, 1.5$ equiv), ethyl acrylate (44 $\mu \mathrm{L}, 0.40 \mathrm{mmol}, 2.0$ equiv), $\mathrm{Pd}_{2}(\mathrm{dba})_{3}(9.2 \mathrm{mg}, 5 \mathrm{~mol} \%)$, TFP (10.2 mg, $\left.22 \mathrm{~mol} \%\right), \mathbf{N 2}$ (71 $\mu \mathrm{L}, 0.60 \mathrm{mmol}, 3.0$ equiv), $\mathrm{K}_{2} \mathrm{CO}_{3}(82.9 \mathrm{mg}, 0.60 \mathrm{mmol}, 3.0$ equiv) in THF ( $2.0 \mathrm{~mL})$ delivered 3o (51.7 mg, 76\%) (1/150 ethyl acetate/hexanes). ${ }^{1} \mathbf{H}$ NMR (400 MHz, $\left.\mathrm{CDCl}_{3}\right) \delta 8.28(\mathrm{~d}, J=16.4$ Hz, 1H), $8.17-8.08$ (m, 1H), $7.87-7.79(\mathrm{~m}, 1 \mathrm{H}), 7.75$ (d, $J=8.8 \mathrm{~Hz}, 1 \mathrm{H}), 7.56$ (d, $J=8.4 \mathrm{~Hz}$, 1H), $7.53-7.43(\mathrm{~m}, 2 \mathrm{H}), 6.30(\mathrm{~d}, J=16.4 \mathrm{~Hz}, 1 \mathrm{H}), 4.34(\mathrm{q}, J=7.2 \mathrm{~Hz}, 2 \mathrm{H}), 3.26(\mathrm{tt}, J=10.4,3.6$ $\mathrm{Hz}, 1 \mathrm{H}), 2.03-1.91(\mathrm{~m}, 2 \mathrm{H}), 1.83-1.72(\mathrm{~m}, 2 \mathrm{H}), 1.66-1.58(\mathrm{~m}, 1 \mathrm{H}), 1.50-1.35(\mathrm{~m}, 5 \mathrm{H}), 1.35-$ $1.22(\mathrm{~m}, 3 \mathrm{H}) .{ }^{13} \mathrm{C}$ NMR $\left(100 \mathrm{MHz}, \mathrm{CDCl}_{3}\right) \delta 166.6,141.8,134.2,133.5,132.3,131.5,128.8,128.6$, 128.3, 127.0, 125.9, 125.8, 125.0, 60.6, 47.1, 33.3, 26.0, 25.7, 14.3. HRMS (ESI) Calcd for $\mathrm{C}_{21} \mathrm{H}_{25} \mathrm{O}_{2} \mathrm{~S}[\mathrm{M}+\mathrm{H}]^{+}$341.1575, found 341.1569. 
Synthesis of compound $\mathbf{3 p}$

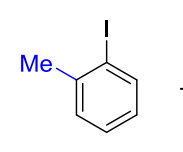

$1 f$

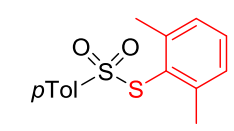

2j

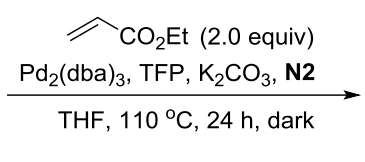

THF, $110^{\circ} \mathrm{C}, 24 \mathrm{~h}$, dark

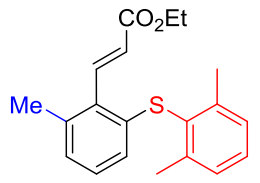

$3 p$

The reaction of 2-iodotoluene $\mathbf{1 f}(43.6 \mathrm{mg}, 0.20 \mathrm{mmol}, 1.0$ equiv), $\mathbf{2 j}$ ( $87.7 \mathrm{mg}, 0.30 \mathrm{mmol}, 1.5$ equiv), ethyl acrylate ( $44 \mu \mathrm{L}, 0.40 \mathrm{mmol}, 2.0$ equiv), $\mathrm{Pd}_{2}(\mathrm{dba})_{3}(9.2 \mathrm{mg}, 5 \mathrm{~mol} \%)$, TFP (10.2 mg, $22 \mathrm{~mol} \%), \mathbf{N 2}\left(71 \mu \mathrm{L}, 0.60 \mathrm{mmol}, 3.0\right.$ equiv), $\mathrm{K}_{2} \mathrm{CO}_{3}(82.9 \mathrm{mg}, 0.60 \mathrm{mmol}, 3.0$ equiv) in THF (2.0 $\mathrm{mL}$ ) delivered 3p (33.0 mg, 50\%) (1/200 ethyl acetate/hexanes). ${ }^{1} \mathbf{H}$ NMR (400 MHz, $\left.\mathrm{CDCl}_{3}\right) \delta$ $7.98(\mathrm{~d}, J=16.0 \mathrm{~Hz}, 1 \mathrm{H}), 7.24(\mathrm{dd}, J=8.6,6.2 \mathrm{~Hz}, 1 \mathrm{H}), 7.20-7.14(\mathrm{~m}, 2 \mathrm{H}), 7.00-6.86(\mathrm{~m}, 2 \mathrm{H})$, $6.42-6.32(\mathrm{~m}, 1 \mathrm{H}), 6.30(\mathrm{~d}, J=16.4 \mathrm{~Hz}, 1 \mathrm{H}), 4.31(\mathrm{q}, J=7.2 \mathrm{~Hz}, 2 \mathrm{H}), 2.38(\mathrm{~s}, 6 \mathrm{H}), 2.37(\mathrm{~s}, 3 \mathrm{H})$, $1.37(\mathrm{t}, J=7.2 \mathrm{~Hz}, 3 \mathrm{H}) .{ }^{13} \mathbf{C} \mathbf{N M R}\left(100 \mathrm{MHz}, \mathrm{CDCl}_{3}\right) \delta 166.7,143.8,141.3,138.4,137.2,132.2$, 130.8, 129.3, 128.6, 128.5, 127.0, 125.0, 122.8, 60.6, 21.8, 21.1, 14.3. HRMS (ESI) Calcd for $\mathrm{C}_{20} \mathrm{H}_{23} \mathrm{O}_{2} \mathrm{~S}[\mathrm{M}+\mathrm{H}]^{+}$327.1419, found 327.1417.

Synthesis of compound $\mathbf{3 q}$

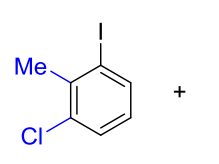

$1 \mathrm{~g}$

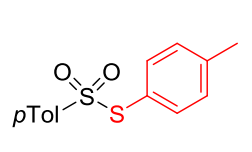

$2 a$

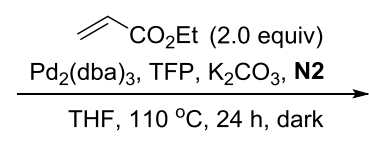

THF, $110^{\circ} \mathrm{C}, 24 \mathrm{~h}$, dark

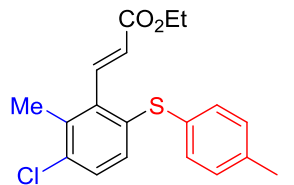

$3 q$

The reaction of $1 \mathrm{~g}$ (50.5 $\mathrm{mg}, 0.20 \mathrm{mmol}, 1.0$ equiv), $2 \mathrm{a}(83.5 \mathrm{mg}, 0.30 \mathrm{mmol}, 1.5$ equiv), ethyl acrylate (44 $\mu \mathrm{L}, 0.40 \mathrm{mmol}, 2.0$ equiv), $\mathrm{Pd}_{2}(\mathrm{dba})_{3}(9.2 \mathrm{mg}, 5 \mathrm{~mol} \%)$, TFP (10.2 mg, $\left.22 \mathrm{~mol} \%\right), \mathbf{N 2}$ (71 $\mu \mathrm{L}, 0.60 \mathrm{mmol}, 3.0$ equiv), $\mathrm{K}_{2} \mathrm{CO}_{3}(82.9 \mathrm{mg}, 0.60 \mathrm{mmol}, 3.0$ equiv) in THF ( $2.0 \mathrm{~mL})$ delivered 3q (41.3 mg, 60\%) (1/150 ethyl acetate/hexanes). ${ }^{1} \mathbf{H}$ NMR (400 MHz, $\left.\mathrm{CDCl}_{3}\right) \delta 7.83$ (d, $J=16.0$ $\mathrm{Hz}, 1 \mathrm{H}), 7.26-7.21(\mathrm{~m}, 2 \mathrm{H}), 7.17(\mathrm{~d}, J=8.8 \mathrm{~Hz}, 1 \mathrm{H}), 7.16-7.10(\mathrm{~m}, 2 \mathrm{H}), 6.87(\mathrm{~d}, J=8.8 \mathrm{~Hz}$, 1H), 6.05 (d, $J=16.4 \mathrm{~Hz}, 1 \mathrm{H}), 4.27$ (q, $J=7.2 \mathrm{~Hz}, 2 \mathrm{H}), 2.38$ (s, 3H), 2.34 (s, 3H), 1.34 (t, $J=7.2$ $\mathrm{Hz}, 3 \mathrm{H}) .{ }^{13} \mathrm{C}$ NMR $\left(100 \mathrm{MHz}, \mathrm{CDCl}_{3}\right) \delta 166.0,141.6,138.2,136.4,136.0,134.7,133.4,132.9$, 130.3, 130.2, 129.3, 128.4, 126.3, 60.7, 21.2, 18.1, 14.3. HRMS (ESI) Calcd for $\mathrm{C}_{19} \mathrm{H}_{20} \mathrm{O}_{2} \mathrm{SCl}[\mathrm{M}+$ $\mathrm{H}]^{+}$347.0873, found 347.0862 . 
Synthesis of compound $\mathbf{3 r}$

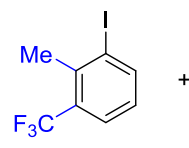

1h

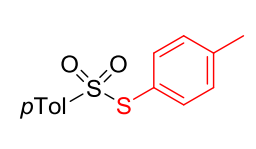

$2 a$

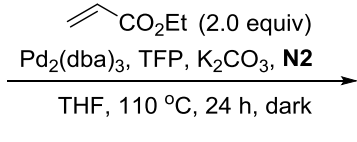

THF, $110^{\circ} \mathrm{C}, 24 \mathrm{~h}$, dark

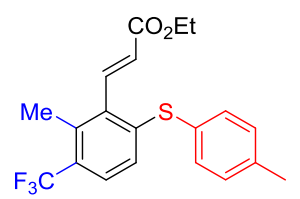

$3 r$

The reaction of $\mathbf{1 h}(57.2 \mathrm{mg}, 0.2 \mathrm{mmol}, 1.0$ equiv), $\mathbf{2 a}(83.5 \mathrm{mg}, 0.3 \mathrm{mmol}, 1.5$ equiv), ethyl acrylate (44 $\mu \mathrm{L}, 0.4 \mathrm{mmol}, 2.0$ equiv), $\mathrm{Pd}_{2}(\mathrm{dba})_{3}(9.2 \mathrm{mg}, 5 \mathrm{~mol} \%)$, TFP (10.2 mg, $\left.22 \mathrm{~mol} \%\right), \mathbf{N 2}$ ( $71 \mu \mathrm{L}, 0.60 \mathrm{mmol}, 3.0$ equiv), $\mathrm{K}_{2} \mathrm{CO}_{3}(82.9 \mathrm{mg}, 0.60 \mathrm{mmol}, 3.0$ equiv) in THF (2.0 mL) delivered 3r (36.4 mg, 47\%) (1/200 ethyl acetate/hexanes). ${ }^{1} \mathbf{H}$ NMR (400 MHz, $\left.\mathrm{CDCl}_{3}\right) \delta 7.80$ (d, $J=16.0$ $\mathrm{Hz}, 1 \mathrm{H}), 7.39-7.32(\mathrm{~m}, 3 \mathrm{H}), 7.25-7.18(\mathrm{~m}, 2 \mathrm{H}), 6.75(\mathrm{~d}, J=8.4 \mathrm{~Hz}, 1 \mathrm{H}), 6.16(\mathrm{~d}, J=16.4 \mathrm{~Hz}$, 1H), $4.30(\mathrm{q}, J=7.2 \mathrm{~Hz}, 2 \mathrm{H}), 2.41(\mathrm{q}, J=1.6 \mathrm{~Hz}, 3 \mathrm{H}), 2.39(\mathrm{~s}, 3 \mathrm{H}), 1.36(\mathrm{t}, J=7.2 \mathrm{~Hz}, 3 \mathrm{H}) .{ }^{13} \mathrm{C}$ $\operatorname{NMR}\left(100 \mathrm{MHz}, \mathrm{CDCl}_{3}\right) \delta 165.9,143.70,143.69,140.8,139.5,135.3(\mathrm{~d}, J=1.4 \mathrm{~Hz}), 134.8,134.7$, 130.6, 128.0, 127.2, $126.3(\mathrm{q}, J=29.5 \mathrm{~Hz}), 125.6(\mathrm{t}, J=5.9 \mathrm{~Hz}), 124.4(\mathrm{t}, J=271.7 \mathrm{~Hz}), 124.2$, 60.8, 21.3, $16.7(\mathrm{q}, J=2.3 \mathrm{~Hz}), 14.3 .{ }^{19} \mathbf{F}$ NMR $\left(376 \mathrm{MHz}, \mathrm{CDCl}_{3}\right) \delta-60.58$. HRMS (ESI) Calcd for $\mathrm{C}_{20} \mathrm{H}_{19} \mathrm{O}_{2} \mathrm{SF}_{3} \mathrm{Na}[\mathrm{M}+\mathrm{Na}]^{+}$403.0956, found 403.0954.

Synthesis of compound $\mathbf{3 s}$

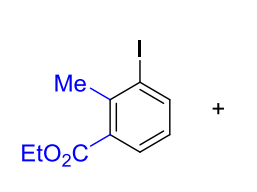

$1 \mathbf{i}$

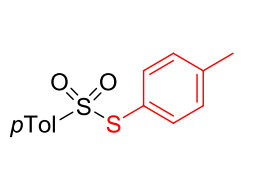

$2 a$
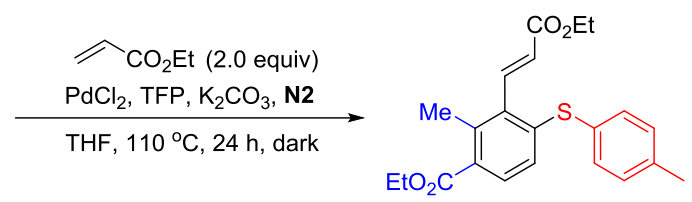

3s

The reaction of $\mathbf{1 i}(57.2 \mathrm{mg}, 0.20 \mathrm{mmol}, 1.0$ equiv), $2 \mathbf{a}(83.5 \mathrm{mg}, 0.30 \mathrm{mmol}, 1.5$ equiv), ethyl acrylate ( $44 \mu \mathrm{L}, 0.40 \mathrm{mmol}, 2.0$ equiv), $\mathrm{PdCl}_{2}(3.5 \mathrm{mg}, 10 \mathrm{~mol} \%)$, TFP (10.2 mg, $\left.22 \mathrm{~mol} \%\right), \mathbf{N 2}(71$ $\mu \mathrm{L}, 0.60 \mathrm{mmol}$, 3.0 equiv), $\mathrm{K}_{2} \mathrm{CO}_{3}(82.9 \mathrm{mg}, 0.60 \mathrm{mmol}, 3.0$ equiv) in THF (2.0 mL) delivered $3 \mathrm{~s}$ $(51.0 \mathrm{mg}, 66 \%)\left(1 / 50\right.$ ethyl acetate/hexanes). ${ }^{1} \mathbf{H}$ NMR $\left(400 \mathrm{MHz}, \mathrm{CDCl}_{3}\right) \delta 7.84(\mathrm{~d}, J=16.4 \mathrm{~Hz}$, 1H), $7.57(\mathrm{~d}, J=8.8 \mathrm{~Hz}, 1 \mathrm{H}), 7.37-7.30(\mathrm{~m}, 2 \mathrm{H}), 7.22-7.17(\mathrm{~m}, 2 \mathrm{H}), 6.74(\mathrm{~d}, J=8.4 \mathrm{~Hz}, 1 \mathrm{H})$, $6.13(\mathrm{~d}, J=16.4 \mathrm{~Hz}, 1 \mathrm{H}), 4.32(\mathrm{q}, J=7.2 \mathrm{~Hz}, 2 \mathrm{H}), 4.29(\mathrm{q}, J=7.2 \mathrm{~Hz}, 2 \mathrm{H}), 2.52(\mathrm{~s}, 3 \mathrm{H}), 2.38(\mathrm{~s}$, $3 \mathrm{H}), 1.353(\mathrm{t}, J=7.2 \mathrm{~Hz}, 3 \mathrm{H}) .1 .347(\mathrm{t}, J=7.2 \mathrm{~Hz}, 3 \mathrm{H}) .{ }^{13} \mathbf{C} \mathbf{N M R}\left(100 \mathrm{MHz}, \mathrm{CDCl}_{3}\right) \delta$ 167.7, 166.0, 143.3, 141.5, 139.2, 137.8, 134.53, 134.46, 130.5, 130.0, 128.6, 128.5, 126.8, 124.6, 60.9, 60.7, 21.3, 18.4, 14.28, 14.25. HRMS (ESI) Calcd for $\mathrm{C}_{22} \mathrm{H}_{24} \mathrm{O}_{4} \mathrm{SNa}[\mathrm{M}+\mathrm{Na}]^{+} 407.1293$, found 407.1288. 
Synthesis of compound $3 \mathrm{t}$

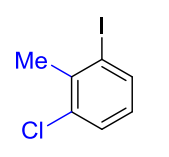

$1 \mathrm{~g}$

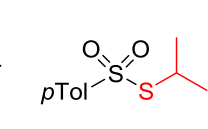

21

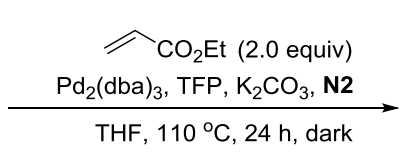

THF, $110^{\circ} \mathrm{C}, 24 \mathrm{~h}$, dark

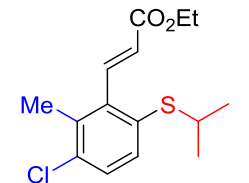

$3 t$

The reaction of $1 \mathrm{~g}$ (50.5 $\mathrm{mg}, 0.20 \mathrm{mmol}, 1.0$ equiv), $2 \mathrm{l}$ ( $69.1 \mathrm{mg}, 0.30 \mathrm{mmol}, 1.5$ equiv), ethyl acrylate (44 $\mu \mathrm{L}, 0.40 \mathrm{mmol}, 2.0$ equiv), $\mathrm{Pd}_{2}(\mathrm{dba})_{3}(9.2 \mathrm{mg}, 5 \mathrm{~mol} \%)$, TFP (10.2 mg, $\left.22 \mathrm{~mol} \%\right), \mathbf{N 2}$ (71 $\mu \mathrm{L}, 0.60 \mathrm{mmol}, 3.0$ equiv), $\mathrm{K}_{2} \mathrm{CO}_{3}(82.9 \mathrm{mg}, 0.60 \mathrm{mmol}, 3.0$ equiv) in THF (2.0 mL) delivered $3 \mathbf{t}$ (33.3 mg, 55\%) (1/200 ethyl acetate/hexanes). $\left.{ }^{1} \mathbf{H} \mathbf{~ N M R ~ ( 4 0 0 ~ M H z , ~} \mathrm{CDCl}_{3}\right) \delta 7.89$ (d, $J=16.0$ $\mathrm{Hz}, 1 \mathrm{H}), 7.28(\mathrm{~d}, J=8.4 \mathrm{~Hz}, 1 \mathrm{H}), 7.20(\mathrm{~d}, J=8.4 \mathrm{~Hz}, 1 \mathrm{H}), 6.03(\mathrm{~d}, J=16.4 \mathrm{~Hz}, 1 \mathrm{H}), 4.28(\mathrm{q}, J=$ $7.2 \mathrm{~Hz}, 2 \mathrm{H}), 3.31$ (hept, $J=6.8 \mathrm{~Hz}, 1 \mathrm{H}), 2.39$ (s, 3H), $1.35(\mathrm{t}, J=7.2 \mathrm{~Hz}, 3 \mathrm{H}), 1.26(\mathrm{~d}, J=6.8 \mathrm{~Hz}$, 6H). ${ }^{13} \mathbf{C}$ NMR $\left(100 \mathrm{MHz}, \mathrm{CDCl}_{3}\right) \delta 166.2,142.4,138.1,134.7,134.4,133.7,129.5,129.0,125.7$, 60.7, 38.2, 22.9, 18.3, 14.3. HRMS (ESI) Calcd for $\mathrm{C}_{15} \mathrm{H}_{20} \mathrm{O}_{2} \mathrm{SCl}[\mathrm{M}+\mathrm{H}]^{+}$299.0872, found 299.0875 .

Synthesis of compound $\mathbf{3 u}$

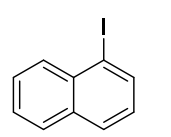

$1 \mathrm{a}$

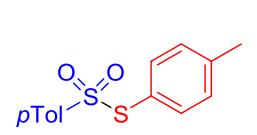

$2 a$

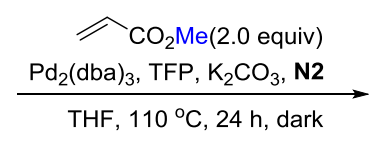

THF, $110^{\circ} \mathrm{C}, 24 \mathrm{~h}$, dark

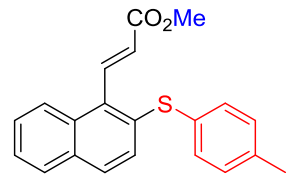

$3 u$

The reaction of $1 \mathbf{a}(50.8 \mathrm{mg}, 0.20 \mathrm{mmol}, 1.0$ equiv), $\mathbf{2 a}(83.5 \mathrm{mg}, 0.30 \mathrm{mmol}, 1.5$ equiv), methyl acrylate (36 $\mu \mathrm{L}, 0.40 \mathrm{mmol}, 2.0$ equiv), $\mathrm{Pd}_{2}(\mathrm{dba})_{3}(9.2 \mathrm{mg}, 5 \mathrm{~mol} \%)$, TFP (10.2 mg, $\left.22 \mathrm{~mol} \%\right)$, N2 (71 $\mu \mathrm{L}, 0.60 \mathrm{mmol}, 3.0$ equiv), $\mathrm{K}_{2} \mathrm{CO}_{3}(82.9 \mathrm{mg}, 0.60 \mathrm{mmol}, 3.0$ equiv) in THF (2.0 mL) delivered 3u (48.2 mg, 72\%) (1/200 ethyl acetate/hexanes). ${ }^{1} \mathbf{H}$ NMR (400 MHz, $\left.\mathrm{CDCl}_{3}\right) \delta 8.29$ (d, $J=16.4$ $\mathrm{Hz}, 1 \mathrm{H}), 8.15-8.08(\mathrm{~m}, 1 \mathrm{H}), 7.82-7.75(\mathrm{~m}, 1 \mathrm{H}), 7.65(\mathrm{~d}, J=8.8 \mathrm{~Hz}, 1 \mathrm{H}), 7.55-7.44(\mathrm{~m}, 2 \mathrm{H})$, $7.31-7.27(\mathrm{~m}, 2 \mathrm{H}), 7.21(\mathrm{~d}, J=8.8 \mathrm{~Hz}, 1 \mathrm{H}), 7.17-7.12(\mathrm{~m}, 2 \mathrm{H}), 6.35(\mathrm{~d}, J=16.4 \mathrm{~Hz}, 1 \mathrm{H}), 3.87$ (s, 3H), 2.36 (s, 3H). ${ }^{13} \mathbf{C}$ NMR (100 MHz, $\left.\mathrm{CDCl}_{3}\right) \delta$ 166.8, 141.2, 138.0, 135.0, 132.6, 132.2, 132.1, 131.4, 131.0, 130.1, 129.2, 128.4, 127.5, 127.2, 125.99, 125.96, 124.7, 51.8, 21.1. HRMS (ESI) Calcd for $\mathrm{C}_{21} \mathrm{H}_{19} \mathrm{O}_{2} \mathrm{~S}[\mathrm{M}+\mathrm{H}]^{+}$335.1106, found 335.1098. 
Synthesis of compound $\mathbf{3 v}$

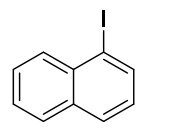

$1 \mathrm{a}$

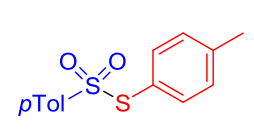

2a

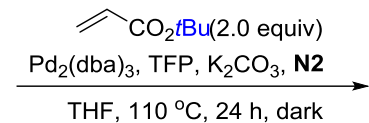

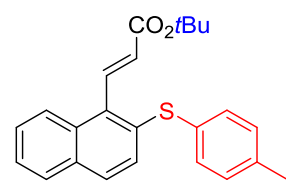

3v

The reaction of $\mathbf{1 a}(50.8 \mathrm{mg}, 0.20 \mathrm{mmol}, 1.0$ equiv), $\mathbf{2 a}(83.5 \mathrm{mg}, 0.30 \mathrm{mmol}, 1.5$ equiv), tertbutyl acrylate (58 $\mu \mathrm{L}, 0.40 \mathrm{mmol}, 2.0$ equiv), $\mathrm{Pd}_{2}(\mathrm{dba})_{3}(9.2 \mathrm{mg}, 5 \mathrm{~mol} \%)$, TFP (10.2 mg, $22 \mathrm{~mol} \%$ ), N2 (71 $\mu \mathrm{L}, 0.60 \mathrm{mmol}, 3.0$ equiv), $\mathrm{K}_{2} \mathrm{CO}_{3}(82.9 \mathrm{mg}, 0.60 \mathrm{mmol}, 3.0$ equiv) in THF (2.0 mL) delivered 3v (45.5 mg, 60\%) (1/200 ethyl acetate/hexanes). ${ }^{1} \mathbf{H}$ NMR (400 MHz, $\left.\mathrm{CDCl}_{3}\right) \delta 8.22-8.11$ $(\mathrm{m}, 2 \mathrm{H}), 7.81-7.74(\mathrm{~m}, 1 \mathrm{H}), 7.64(\mathrm{~d}, J=8.8 \mathrm{~Hz}, 1 \mathrm{H}), 7.56-7.42(\mathrm{~m}, 2 \mathrm{H}), 7.34-7.27(\mathrm{~m}, 2 \mathrm{H})$, $7.22(\mathrm{~d}, J=8.4 \mathrm{~Hz}, 1 \mathrm{H}), 7.17-7.10(\mathrm{~m}, 2 \mathrm{H}), 6.24(\mathrm{~d}, J=16.0 \mathrm{~Hz}, 1 \mathrm{H}), 2.35(\mathrm{~s}, 3 \mathrm{H}), 1.58(\mathrm{~s}, 9 \mathrm{H})$. ${ }^{13}$ C NMR $\left(100 \mathrm{MHz}, \mathrm{CDCl}_{3}\right) \delta 165.7,139.8,137.9,134.8,132.6,132.4,132.2,131.5,131.2,130.1$, 128.9, 128.4, 128.2, 127.6, 127.1, 125.9, 124.9, 80.7, 28.2, 21.2. HRMS (ESI) Calcd for $\mathrm{C}_{24} \mathrm{H}_{25} \mathrm{O}_{2} \mathrm{~S}$ $[\mathrm{M}+\mathrm{H}]^{+}$377.1575, found 377.1577.

Synthesis of compound $\mathbf{3 w}$

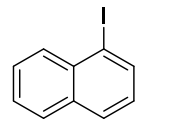

1a

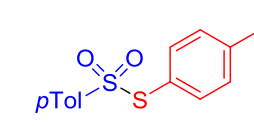

2a

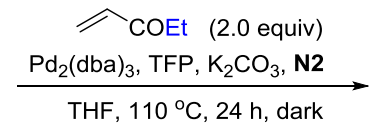

THF, $110^{\circ} \mathrm{C}, 24 \mathrm{~h}$, dark

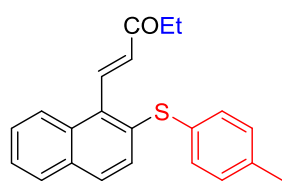

$3 w$

The reaction of $\mathbf{1 a}$ ( $50.8 \mathrm{mg}, 0.20 \mathrm{mmol}, 1.0$ equiv), $\mathbf{2 a}(83.5 \mathrm{mg}, 0.30 \mathrm{mmol}, 1.5$ equiv), ethyl vinylketone (39 $\mu \mathrm{L}, 0.40 \mathrm{mmol}, 2.0$ equiv), $\mathrm{Pd}_{2}(\mathrm{dba})_{3}(9.2 \mathrm{mg}, 5 \mathrm{~mol} \%)$, TFP (10.2 mg, $\left.22 \mathrm{~mol} \%\right)$, N2 (71 $\mu \mathrm{L}, 0.60 \mathrm{mmol}, 3.0$ equiv), $\mathrm{K}_{2} \mathrm{CO}_{3}(82.9 \mathrm{mg}, 0.60 \mathrm{mmol}, 3.0$ equiv) in THF (2.0 mL) delivered 3w (41.3 mg, 62\%) (1/200 ethyl acetate/hexanes). ${ }^{1} \mathbf{H}$ NMR (400 MHz, $\left.\mathrm{CDCl}_{3}\right) \delta 8.14(\mathrm{~d}, J=$ $16.4 \mathrm{~Hz}, 1 \mathrm{H}), 8.12-8.08(\mathrm{~m}, 1 \mathrm{H}), 7.83-7.76(\mathrm{~m}, 1 \mathrm{H}), 7.67(\mathrm{~d}, J=8.8 \mathrm{~Hz}, 1 \mathrm{H}), 7.55-7.44(\mathrm{~m}$, 2H), $7.30-7.26(\mathrm{~m}, 2 \mathrm{H}), 7.23(\mathrm{~d}, J=8.8 \mathrm{~Hz}, 1 \mathrm{H}), 7.18-7.11(\mathrm{~m}, 2 \mathrm{H}), 6.59(\mathrm{~d}, J=16.4 \mathrm{~Hz}, 1 \mathrm{H})$, $2.79(\mathrm{q}, J=7.2 \mathrm{~Hz}, 2 \mathrm{H}), 2.35(\mathrm{~s}, 3 \mathrm{H}), 1.22(\mathrm{t}, J=7.2 \mathrm{~Hz}, 3 \mathrm{H}) .{ }^{13} \mathbf{C} \mathbf{N M R}\left(100 \mathrm{MHz}, \mathrm{CDCl}_{3}\right) \delta 200.7$, $138.5,137.9,134.9,134.2,132.5,132.4,132.3,131.4,131.0,130.1,129.2,128.5,127.6,127.2$, 126.0, 124.8, 33.7, 21.1, 8.2. HRMS (ESI) Calcd for $\mathrm{C}_{22} \mathrm{H}_{21} \mathrm{OS}[\mathrm{M}+\mathrm{H}]^{+} 333.1313$, found 333.1306.

Synthesis of compound $\mathbf{3 x}$ 

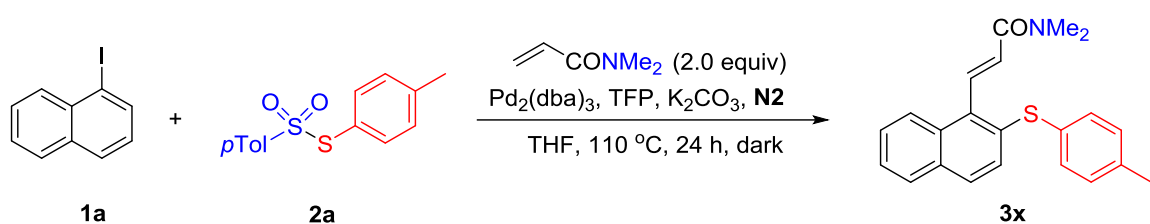

$3 \mathbf{x}$

The reaction of $\mathbf{1 a}(50.8 \mathrm{mg}, 0.20 \mathrm{mmol}, 1.0$ equiv), $\mathbf{2 a}(83.5 \mathrm{mg}, 0.30 \mathrm{mmol}, 1.5$ equiv), $\mathrm{N}, \mathrm{N}-$ dimethylacrylamide (41 $\mu \mathrm{L}, 0.40 \mathrm{mmol}, 2.0$ equiv), $\mathrm{Pd}_{2}(\mathrm{dba})_{3}(9.2 \mathrm{mg}, 5 \mathrm{~mol} \%)$, TFP (10.2 mg, 22 mol\%), $\mathbf{N} 2\left(71 \mu \mathrm{L}, 0.60 \mathrm{mmol}, 3.0\right.$ equiv), $\mathrm{K}_{2} \mathrm{CO}_{3}(82.9 \mathrm{mg}, 0.60 \mathrm{mmol}, 3.0$ equiv) in $\mathrm{THF}(2.0 \mathrm{~mL})$ delivered 3x (33.4 mg, 48\%) (1/5 ethyl acetate/hexanes). ${ }^{\mathbf{1}} \mathbf{H} \mathbf{N M R}\left(400 \mathrm{MHz}, \mathrm{CDCl}_{3}\right) \delta 8.16(\mathrm{~d}, J$ $=15.6 \mathrm{~Hz}, 1 \mathrm{H}), 8.14-8.09(\mathrm{~m}, 1 \mathrm{H}), 7.80-7.74(\mathrm{~m}, 1 \mathrm{H}), 7.63(\mathrm{~d}, J=8.8 \mathrm{~Hz}, 1 \mathrm{H}), 7.54-7.42(\mathrm{~m}$, 2H), $7.30-7.24(\mathrm{~m}, 2 \mathrm{H}), 7.22(\mathrm{~d}, J=8.8 \mathrm{~Hz}, 1 \mathrm{H}), 7.16-7.09(\mathrm{~m}, 2 \mathrm{H}), 6.75(\mathrm{~d}, J=15.6 \mathrm{~Hz}, 1 \mathrm{H})$, $3.10(\mathrm{~s}, 6 \mathrm{H}), 2.34(\mathrm{~s}, 3 \mathrm{H}) .{ }^{13} \mathbf{C}$ NMR $\left(100 \mathrm{MHz}, \mathrm{CDCl}_{3}\right) \delta 166.3,138.1,137.7,134.0,133.6,132.4$, $132.1,131.8,131.4,130.1,128.6,128.3,127.8,126.9,126.4,125.9,125.0,37.4,35.8,21.1$. HRMS (ESI) Calcd for $\mathrm{C}_{22} \mathrm{H}_{22} \mathrm{NOS}[\mathrm{M}+\mathrm{H}]^{+} 348.1422$, found 348.1418 .

Synthesis of compound $\mathbf{3 b b}$

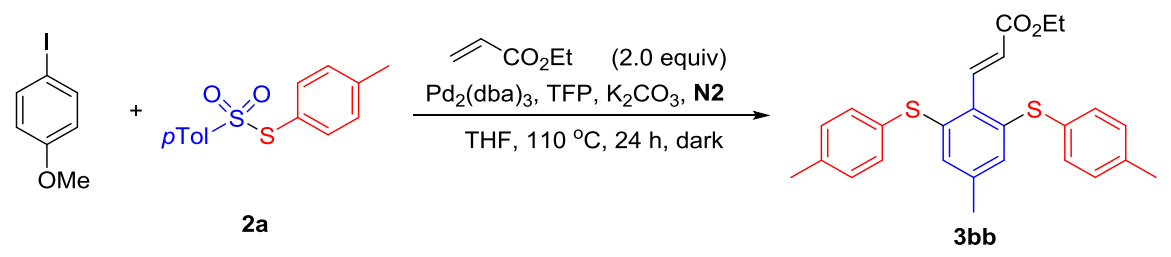

The reaction of 4-Iodoanisole (46.8 mg, $0.20 \mathrm{mmol}, 1.0$ equiv), $\mathbf{2 a}$ (167.0 $\mathrm{mg}, 0.60 \mathrm{mmol}, 3.0$ equiv), ethyl acrylate (44 $\mu \mathrm{L}, 0.40 \mathrm{mmol}, 2.0$ equiv), $\mathrm{Pd}_{2}(\mathrm{dba})_{3}(9.2 \mathrm{mg}, 5 \mathrm{~mol} \%)$, TFP (10.2 mg, $22 \mathrm{~mol} \%), \mathbf{N} 2\left(71 \mu \mathrm{L}, 0.60 \mathrm{mmol}, 3.0\right.$ equiv), $\mathrm{K}_{2} \mathrm{CO}_{3}(110.6 \mathrm{mg}, 0.60 \mathrm{mmol}, 4.0$ equiv) in THF (2.0 $\mathrm{mL}$ ) delivered 3bb (17.2 mg, 13\%)(1/150 ethyl acetate/hexanes). The same product was detected by TLC for both reactions of 4-iodotoluene and 2-iodoanisole. ${ }^{1} \mathrm{H}$ NMR (400 MHz, $\left.\mathrm{CDCl}_{3}\right) \delta 7.93$ $(\mathrm{d}, J=16.4 \mathrm{~Hz}, 1 \mathrm{H}), 7.30-7.25(\mathrm{~m}, 4 \mathrm{H}), 7.17-7.11(\mathrm{~m}, 4 \mathrm{H}), 6.73(\mathrm{~s}, 2 \mathrm{H}), 6.33(\mathrm{~d}, J=16.4 \mathrm{~Hz}$, 1H), $4.24(\mathrm{q}, J=7.2 \mathrm{~Hz}, 2 \mathrm{H}), 2.35(\mathrm{~s}, 6 \mathrm{H}), 2.08(\mathrm{~s}, 3 \mathrm{H}), 1.32(\mathrm{t}, J=7.2 \mathrm{~Hz}, 3 \mathrm{H}) .{ }^{13} \mathrm{C}$ NMR $(100$ $\left.\mathrm{MHz}, \mathrm{CDCl}_{3}\right) \delta 166.4,140.3,139.1,138.3,138.1,133.0,131.4,130.4,130.2,128.6,125.6,60.5$, 21.19, 21.17, 14.3. HRMS (ESI) Calcd for $\mathrm{C}_{26} \mathrm{H}_{27} \mathrm{O}_{2} \mathrm{~S}_{2}[\mathrm{M}+\mathrm{H}]^{+} 435.1452$, found 435.1449.

Oxidation of sulfide $3 \mathbf{e}$ to sulfoxide $4 \mathbf{e}$. 
<smiles>CCO/C=C\c1c(Sc2ccccc2C)ccc2ccccc12</smiles>

$3 e$

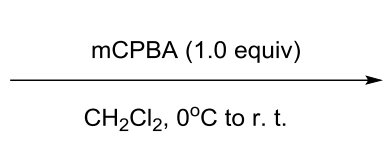

$\mathrm{CH}_{2} \mathrm{Cl}_{2}, 0^{\circ} \mathrm{C}$ to $\mathrm{r}$.

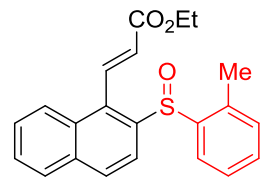

$4 e, 66 \%$

Sulfide 3e $(0.270 \mathrm{~g}, 0.77 \mathrm{mmol})$ was dissolved in DCM $(10 \mathrm{~mL})$ at $0^{\circ} \mathrm{C}$ with stirring, then 3 chloroperoxybenzoic acid ( $75 \%$ purity) $(0.177 \mathrm{~g}, 0.77 \mathrm{mmol})$ was added in one portion. The mixture was stirred at $0^{\circ} \mathrm{C}$ for $30 \mathrm{~min}$ and warmed up to room temperature for $2 \mathrm{~h}$ (TLC monitoring), The solvent was removed under reduced pressure and the residue was purified by column chromatography on silica gel (1/3 ethyl acetate/hexanes) to give $4 \mathbf{e}(0.181 \mathrm{~g}, 66 \%)$ as a white solid. ${ }^{1} \mathbf{H}$ NMR $\left(400 \mathrm{MHz}, \mathrm{CDCl}_{3}\right) \delta 8.22(\mathrm{~d}, J=16.4 \mathrm{~Hz}, 1 \mathrm{H}), 8.11-8.06(\mathrm{~m}, 1 \mathrm{H}), 7.95(\mathrm{~d}, J=8.8 \mathrm{~Hz}, 1 \mathrm{H}), 7.91$ $-7.84(\mathrm{~m}, 2 \mathrm{H}), 7.81(\mathrm{~d}, J=8.8 \mathrm{~Hz}, 1 \mathrm{H}), 7.65-7.56(\mathrm{~m}, 2 \mathrm{H}), 7.42-7.32(\mathrm{~m}, 2 \mathrm{H}), 7.19-7.12(\mathrm{~m}$, $1 \mathrm{H}), 6.36(\mathrm{~d}, J=16.0 \mathrm{~Hz}, 1 \mathrm{H}), 4.34(\mathrm{q}, J=7.2 \mathrm{~Hz}, 2 \mathrm{H}), 2.28(\mathrm{~s}, 3 \mathrm{H}), 1.39(\mathrm{t}, J=7.2 \mathrm{~Hz}, 3 \mathrm{H}) .{ }^{13} \mathrm{C}$ NMR $\left(100 \mathrm{MHz}, \mathrm{CDCl}_{3}\right) \delta 165.5,142.3,139.7,138.5,136.2,134.4,133.5,131.1,130.9,130.6$, 130.4, 128.7, 128.1, 127.7, 127.1, 125.9, 125.6, 121.5, 61.0, 18.8, 14.3. HRMS (ESI) Calcd for $\mathrm{C}_{22} \mathrm{H}_{20} \mathrm{O}_{3} \mathrm{SNa}[\mathrm{M}+\mathrm{Na}]^{+}$387.1031, found 387.1030.

$Z / E$ isomerization of $\mathbf{3 a}$ and $\mathbf{3 m}$

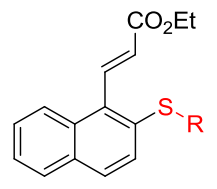

3a, $\mathrm{R}=4-\mathrm{MeC}_{6} \mathrm{H}_{4}$ $3 \mathrm{~m}, \mathrm{R}=i \mathrm{Pr}$

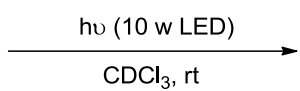

$\mathrm{CDCl}_{3}$,

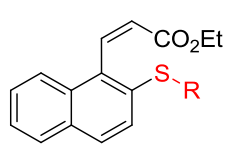

$Z-3 a, 20 h, E \cdot Z=1: 4.5,73 \%$ $\mathrm{Z}-3 \mathrm{~m}, 16 \mathrm{~h}, \mathrm{E}: \mathrm{Z}=1: 3.0,68 \%$

A solution of sulfide $3 \mathrm{~m}$ (42.8 $\mathrm{mg}, 0.142 \mathrm{mmol})$ dissolved in $\mathrm{CDCl}_{3}(1.42 \mathrm{~mL})$ was transferred to a quartz glass tube reactor. The system was degassed with a nitrogen balloon for $15 \mathrm{~min}$, then the reactor was tightly sealed and irradiated by a blue LED lamp $(10 \mathrm{~W}, 465 \mathrm{~nm})$ for $16 \mathrm{~h}$. The $E / Z$ ratio was determined as 1:3.0 by ${ }^{1} \mathrm{H}-\mathrm{NMR}$ spectroscopy, The solvent was removed and the isomers was separated by thin layer chromatography plate (1/80 ethyl acetate/hexanes) to give (Z)-3m (29.2 mg, 68\%). ${ }^{1} \mathbf{H}$ NMR (400 MHz, $\left.\mathrm{CDCl}_{3}\right) \delta 7.88-7.82(\mathrm{~m}, 1 \mathrm{H}), 7.82-7.77(\mathrm{~m}, 1 \mathrm{H}), 7.75(\mathrm{~d}, J=8.4 \mathrm{~Hz}$, $1 \mathrm{H}), 7.54(\mathrm{~d}, J=8.4 \mathrm{~Hz}, 1 \mathrm{H}), 7.47-7.38(\mathrm{~m}, 3 \mathrm{H}), 6.36(\mathrm{~d}, J=11.6 \mathrm{~Hz}, 1 \mathrm{H}), 3.79(\mathrm{q}, J=7.2 \mathrm{~Hz}$, 2H), 3.48 (hept, $J=6.8 \mathrm{~Hz}, 1 \mathrm{H}), 1.29(\mathrm{bs}, 6 \mathrm{H}), 0.69$ (t, $J=7.2 \mathrm{~Hz}, 3 \mathrm{H}) .{ }^{13} \mathrm{C}$ NMR $(100 \mathrm{MHz}$, $\left.\mathrm{CDCl}_{3}\right) \delta 165.5,141.1,136.5,131.9,131.2,130.5,128.8,128.1,127.9,126.5,125.7,125.0,124.6$, 59.9, 38.3, 23.2 (bs), 13.4. HRMS (ESI) Calcd for $\mathrm{C}_{18} \mathrm{H}_{21} \mathrm{O}_{2} \mathrm{~S}[\mathrm{M}+\mathrm{H}]^{+}$301.1262, found 301.1260. 
The reaction of $3 \mathbf{a}(42.2 \mathrm{mg}, 0.121 \mathrm{mmol})$ in $\mathrm{CDCl}_{3}(1.21 \mathrm{~mL})$ for $20 \mathrm{~h}$ resulted a $E / Z$ ratio of 1:4.5 and the (Z)-3a (31.0 mg, 73\%) was obtained by column flash chromatography on silica gel (1/100 ethyl acetate/hexanes). ${ }^{1} \mathbf{H}$ NMR $\left(400 \mathrm{MHz}, \mathrm{CDCl}_{3}\right) \delta 7.90-7.82(\mathrm{~m}, 1 \mathrm{H}), 7.81-7.75(\mathrm{~m}$, 1H), $7.66(\mathrm{~d}, J=8.8 \mathrm{~Hz}, 1 \mathrm{H}), 7.49-7.42(\mathrm{~m}, 2 \mathrm{H}), 7.39(\mathrm{~d}, J=12.0 \mathrm{~Hz}, 1 \mathrm{H}), 7.27(\mathrm{~d}, J=8.4 \mathrm{~Hz}$, 1H), $7.25-7.20(\mathrm{~m}, 2 \mathrm{H}), 7.13-7.07(\mathrm{~m}, 2 \mathrm{H}), 6.36(\mathrm{~d}, J=12.0 \mathrm{~Hz}, 1 \mathrm{H}), 3.84(\mathrm{q}, J=7.2 \mathrm{~Hz}, 2 \mathrm{H})$, $2.33(\mathrm{~s}, 3 \mathrm{H}), 0.78(\mathrm{t}, J=7.2 \mathrm{~Hz}, 3 \mathrm{H}) .{ }^{13} \mathbf{C}$ NMR $\left(100 \mathrm{MHz}, \mathrm{CDCl}_{3}\right) \delta 165.3,140.5,137.2,134.9$, $131.9,131.8,131.6,131.3,131.2,129.9,128.3,128.2,128.0,126.6,125.8,125.2,125.0,60.0,21.1$, 13.5. HRMS (ESI) Calcd for $\mathrm{C}_{22} \mathrm{H}_{21} \mathrm{O}_{2} \mathrm{~S}[\mathrm{M}+\mathrm{H}]^{+} 349.1262$, found 349.1261 .

\section{References}

1. Zhao, X.; Liu, T.-X.; Zhang, G. Asian J. Org. Chem. 2017, 6, 677-681.

2. Fujiki, K.; Tanifuji, N.; Sasaki, Y.; Yokoyama, T. Synthesis, 2002, 343-348.

3. Yoshida, S.; Sugimura, Y.; Hazama, Y.; Nishiyama, Y.; Yano, T.; Shimizub, S.; Hosoya, T. Chem. Commun., 2015, 51, 16613-16616. 


\section{Copies of NMR Spectroscopies}

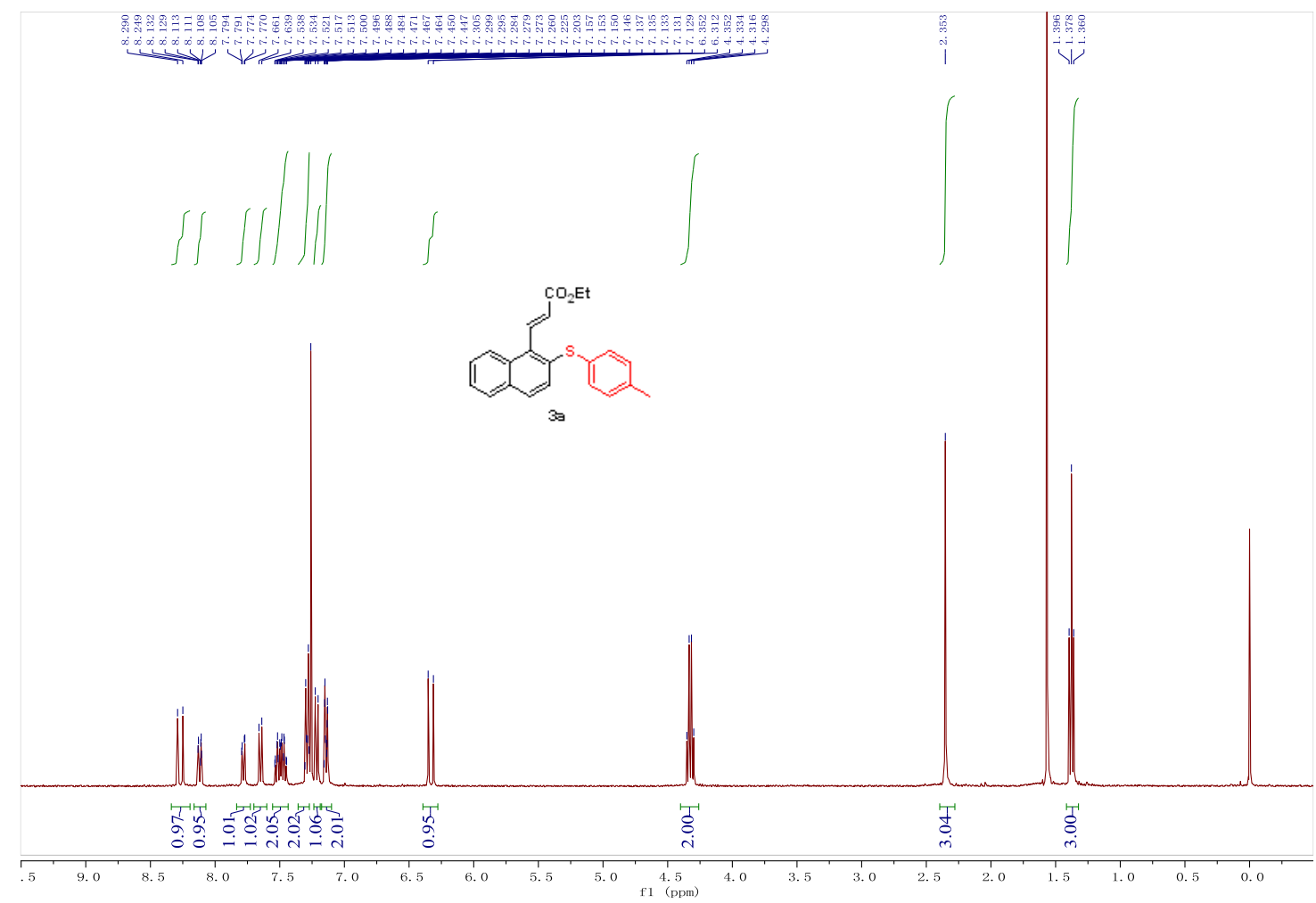

${ }^{1}$ H NMR
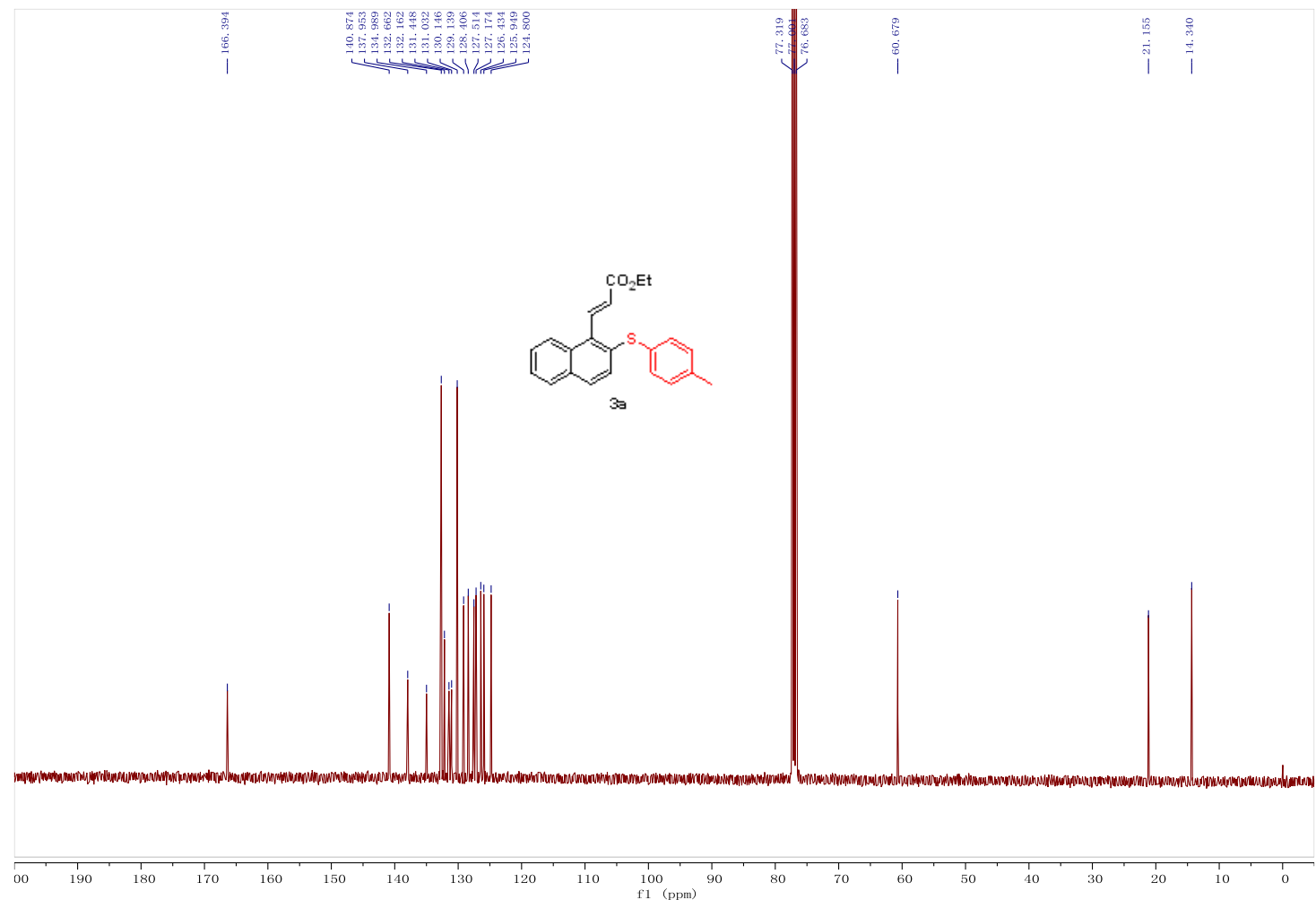

${ }^{13}$ C NMR 


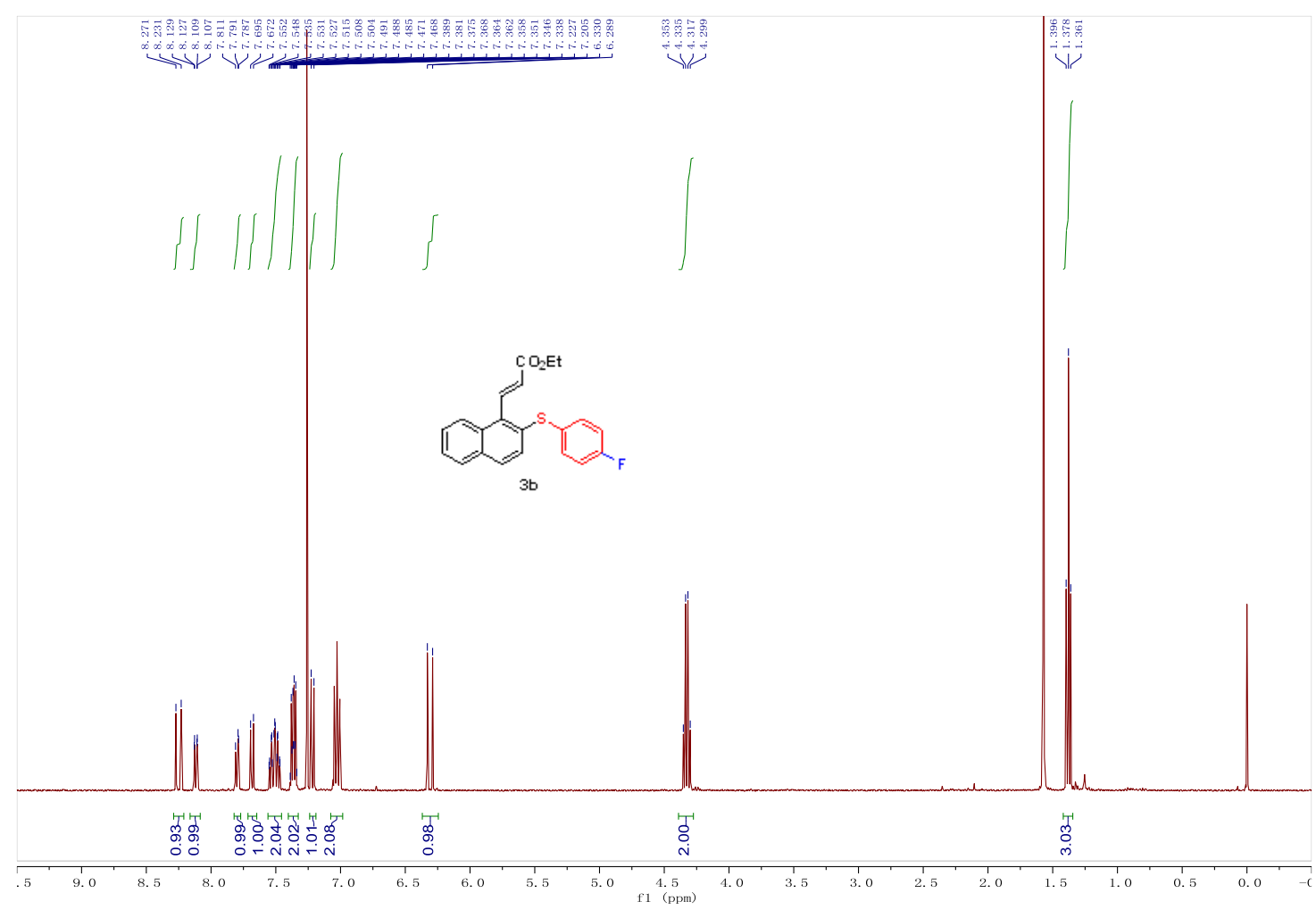

${ }^{1}$ H NMR

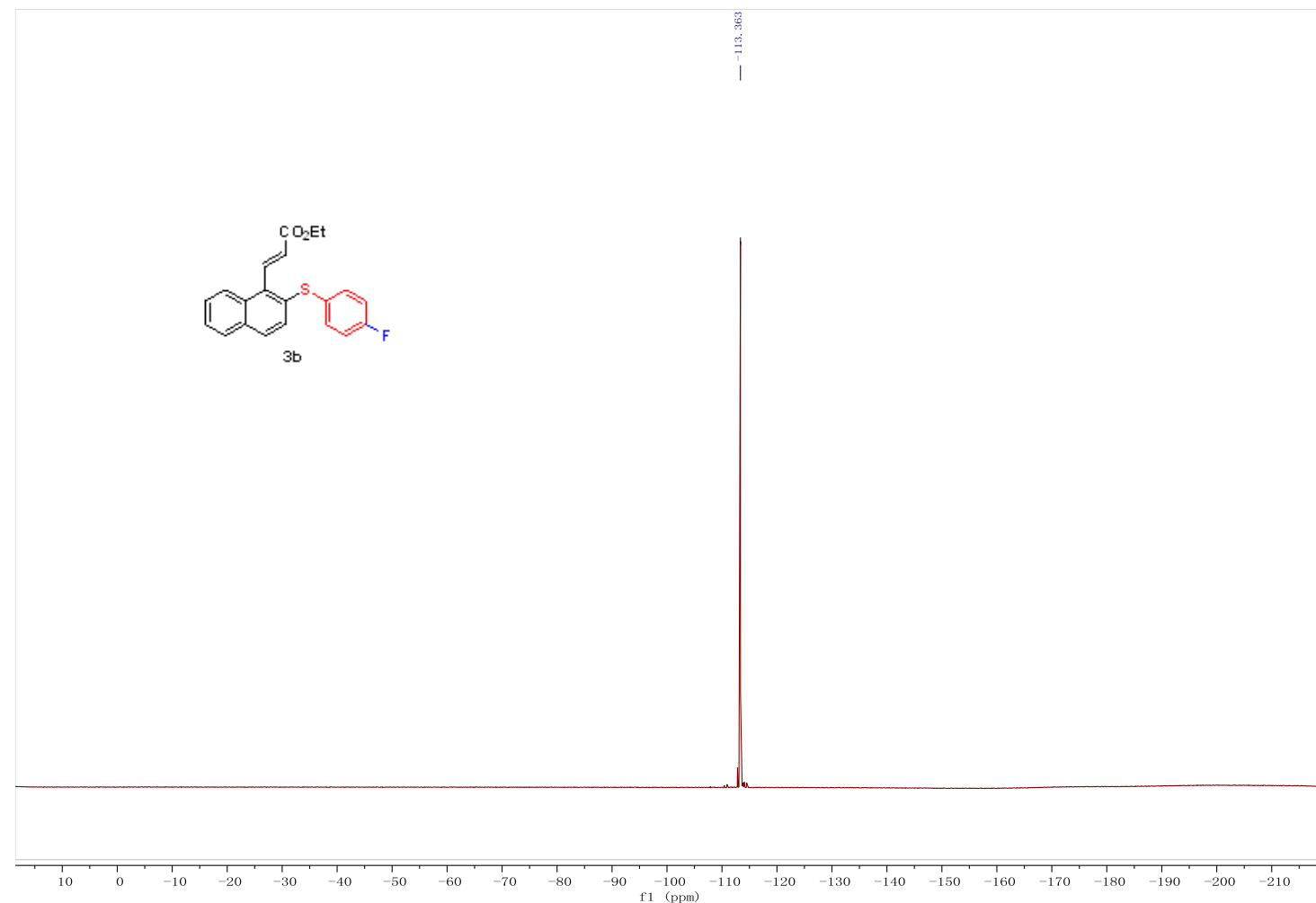

${ }^{19}$ F NMR 

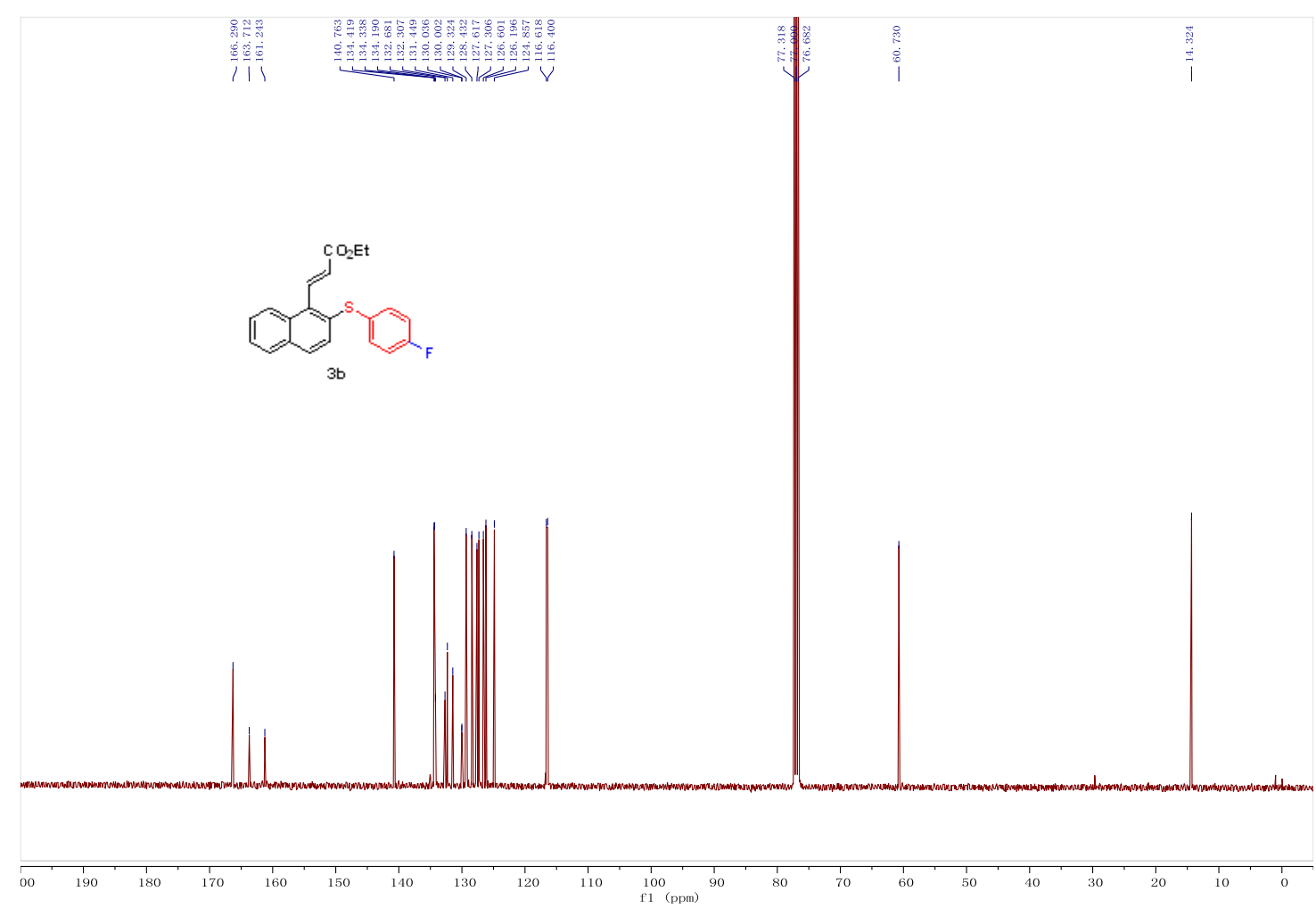

${ }^{13}$ C NMR

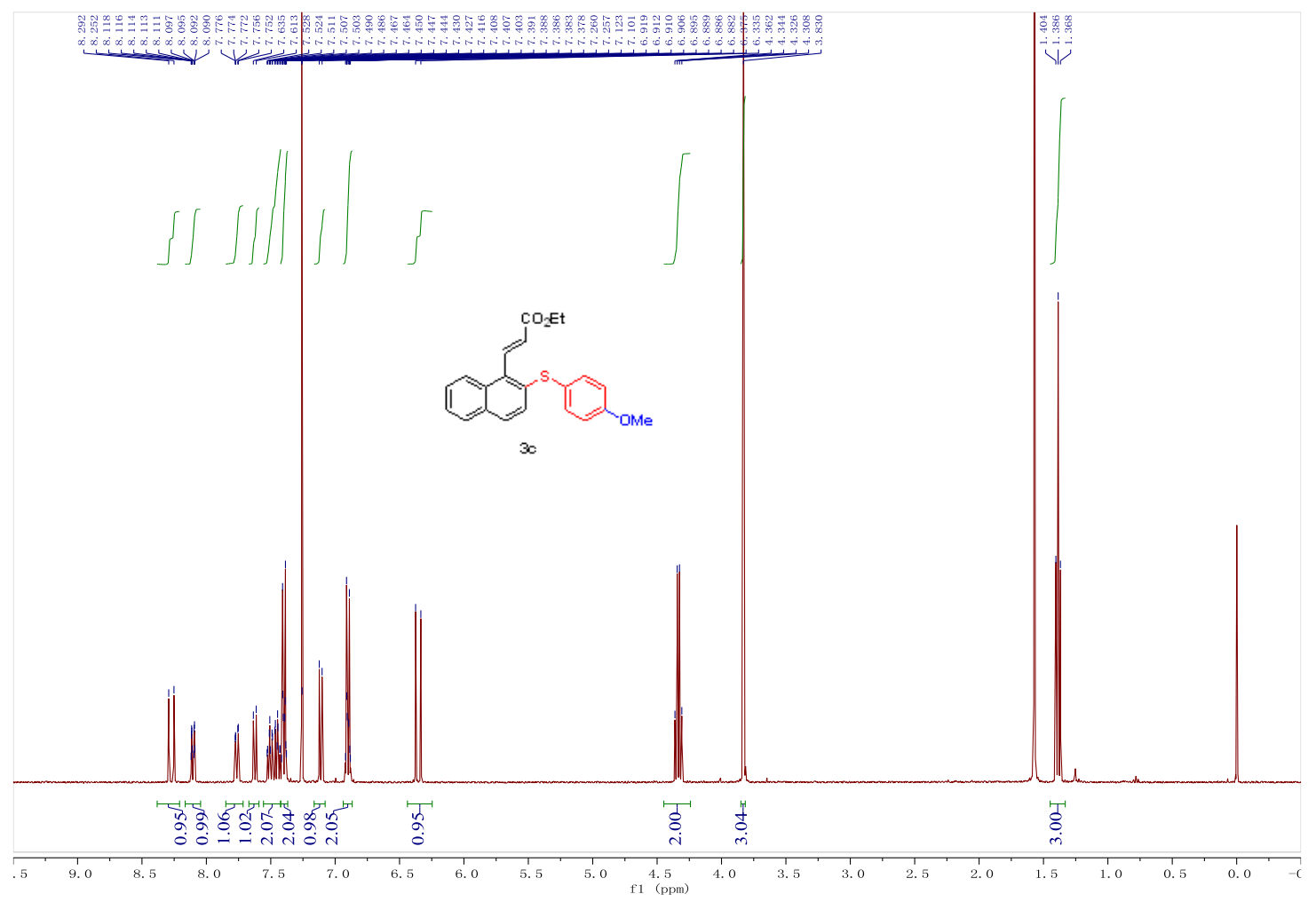

${ }^{1}$ H NMR 

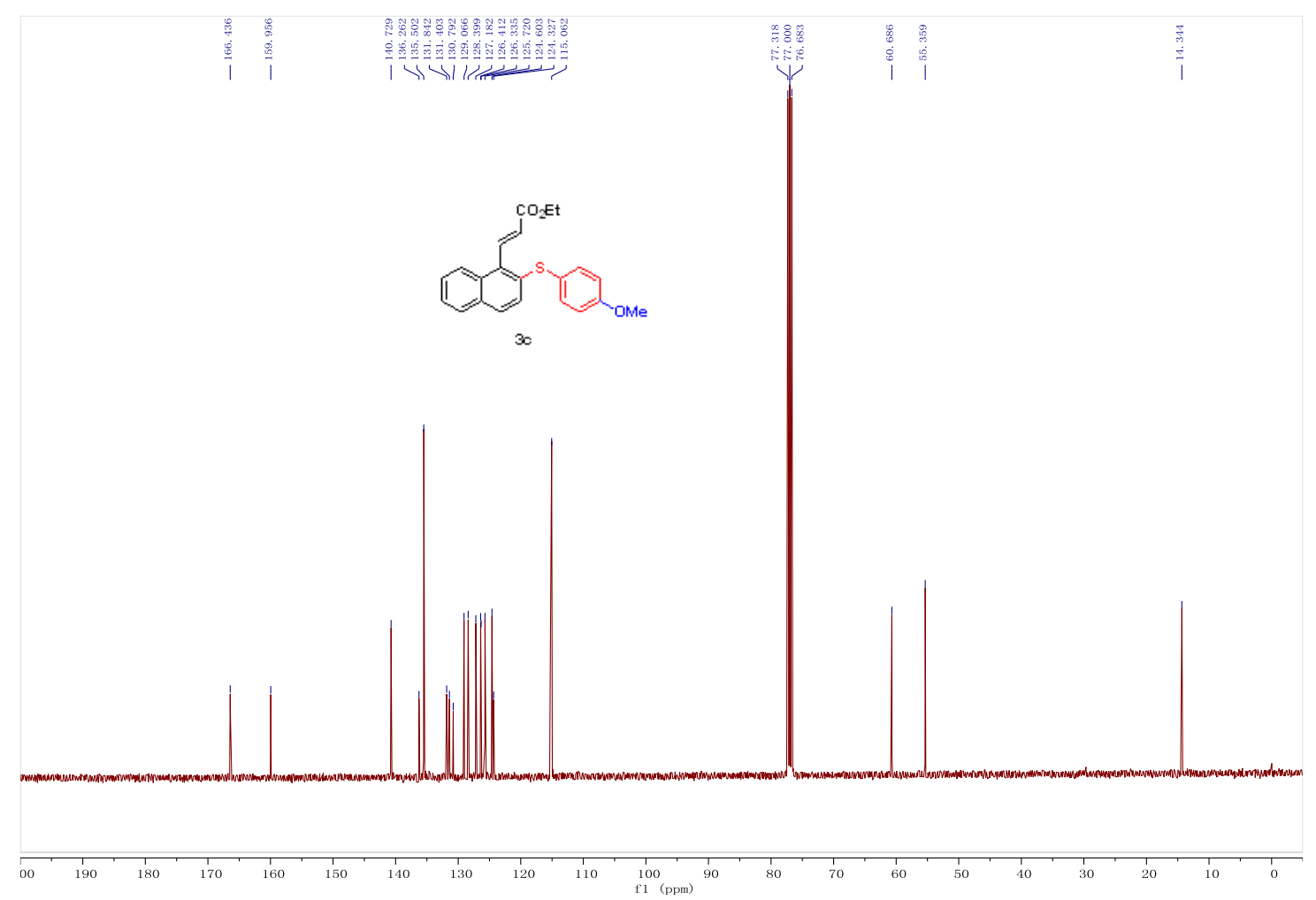

${ }^{13}$ C NMR

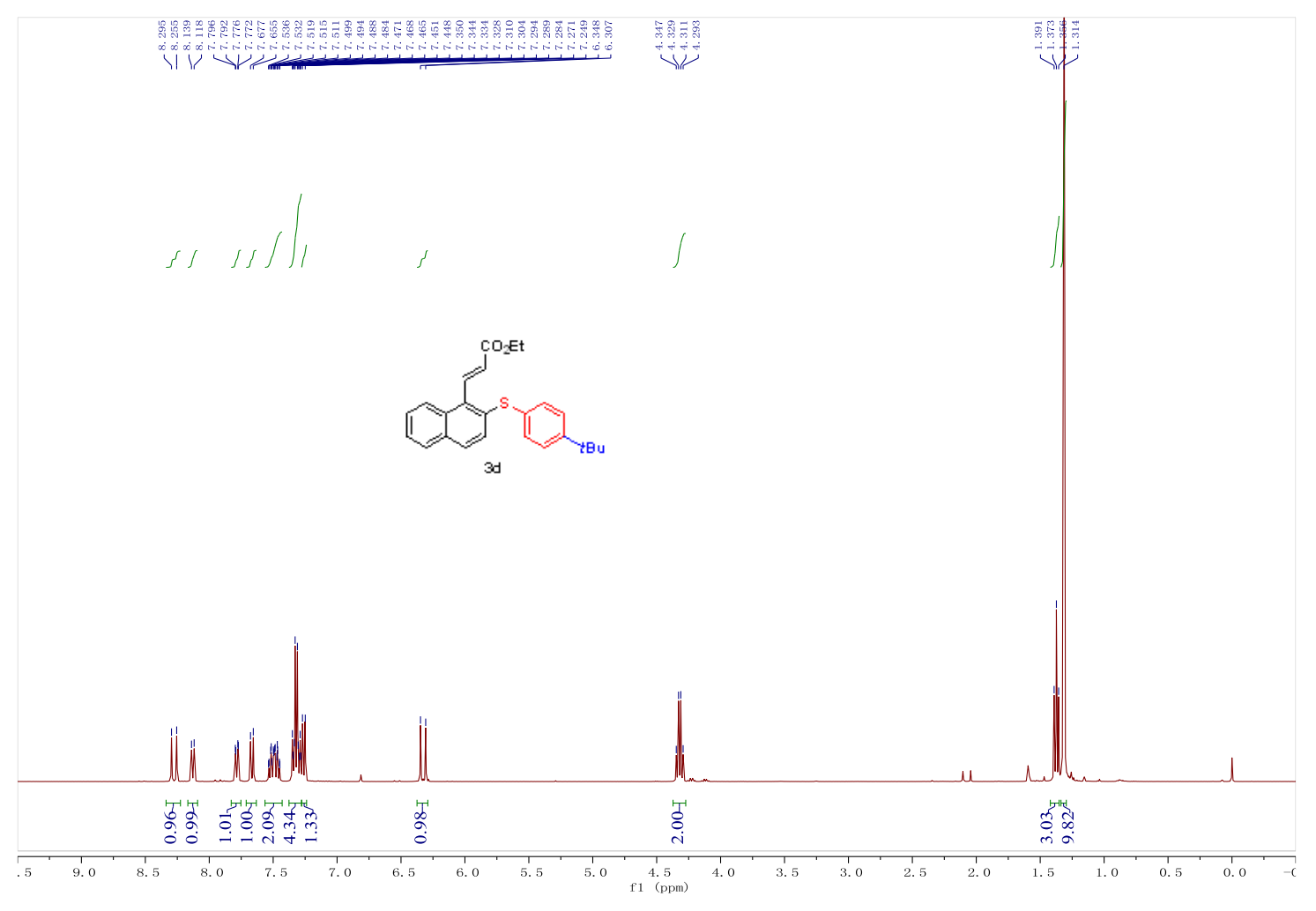

${ }^{1}$ H NMR 

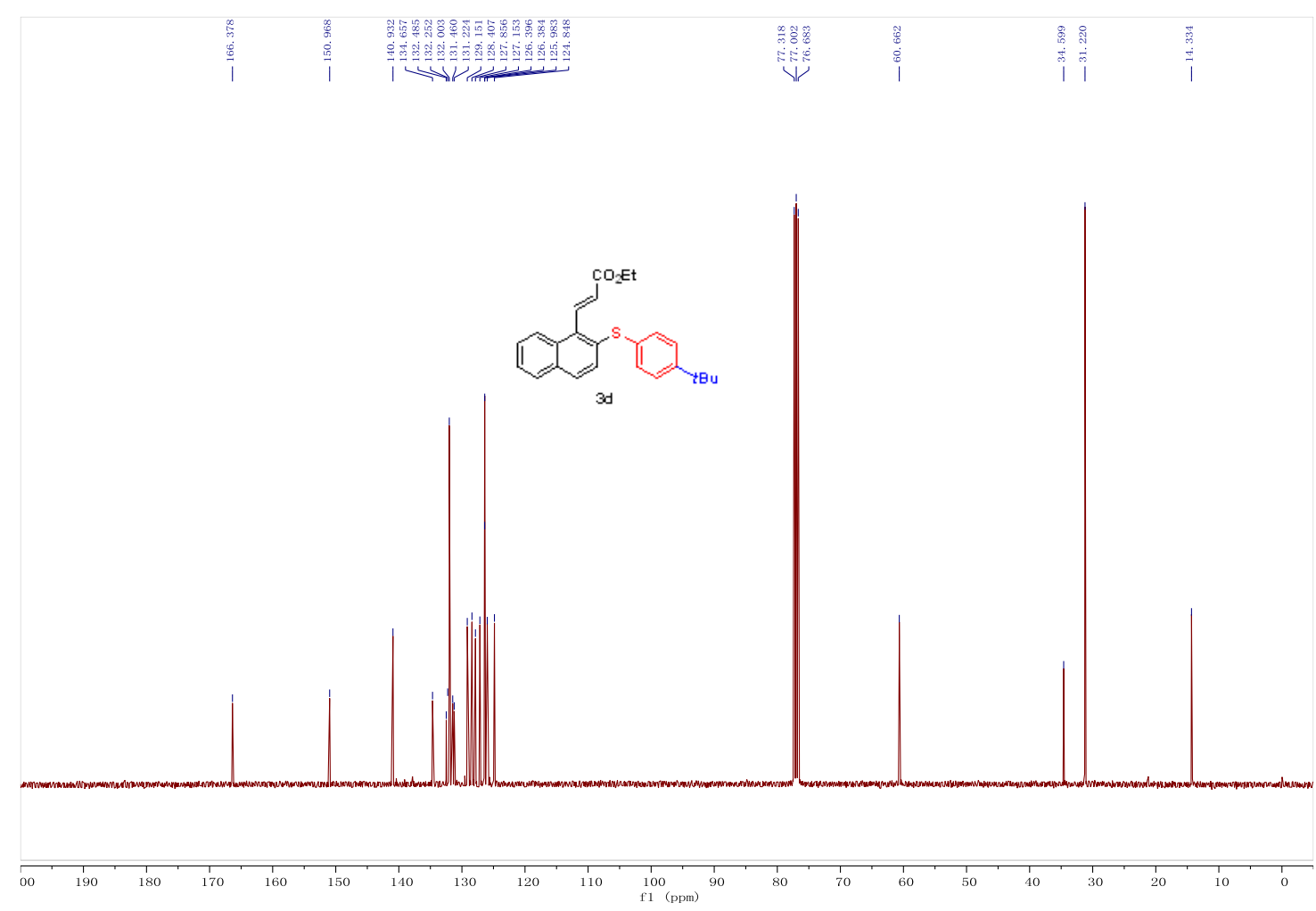

${ }^{13}$ C NMR

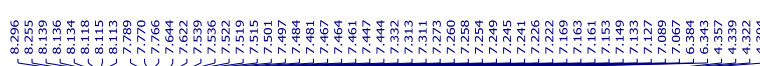
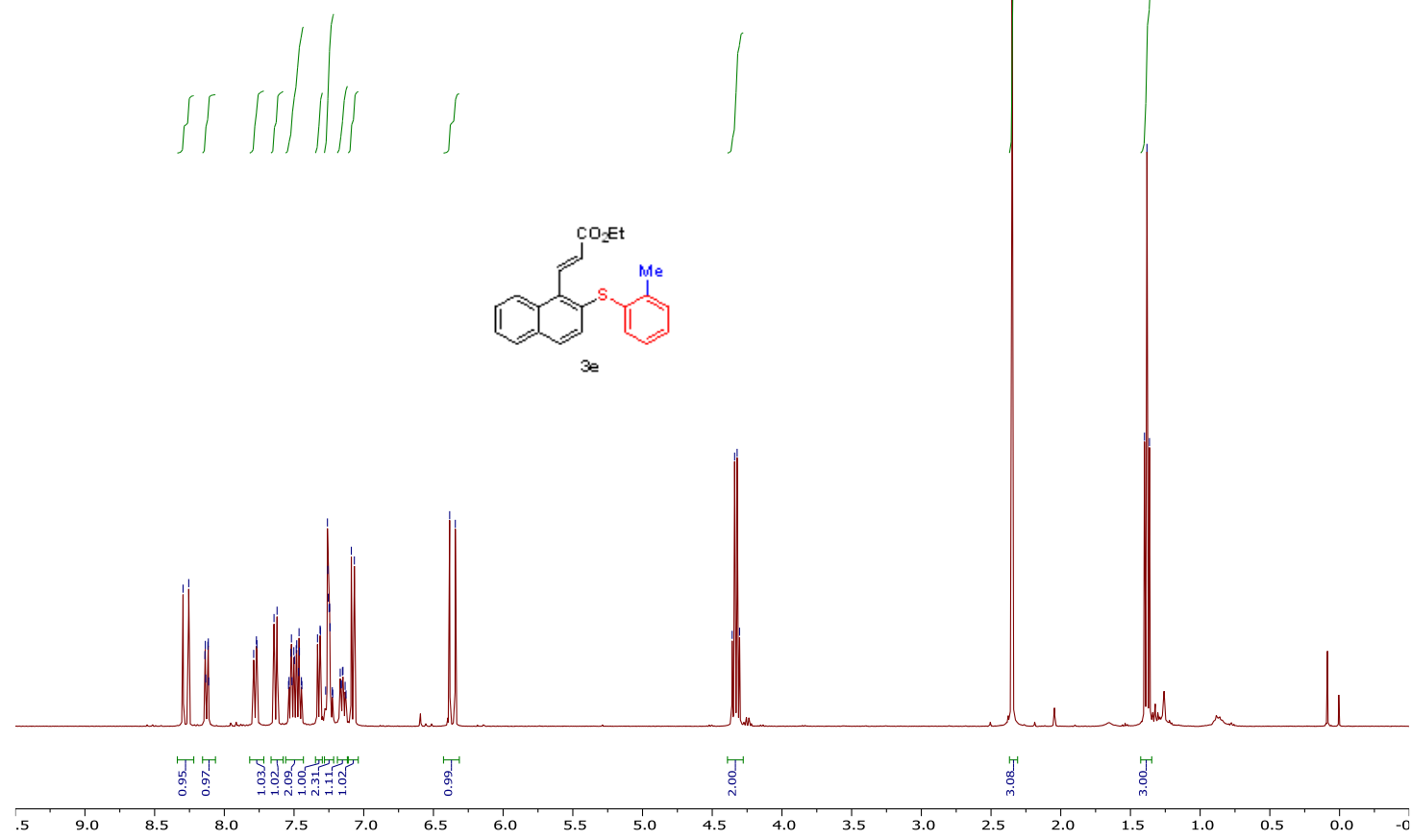

${ }^{1}$ H NMR 


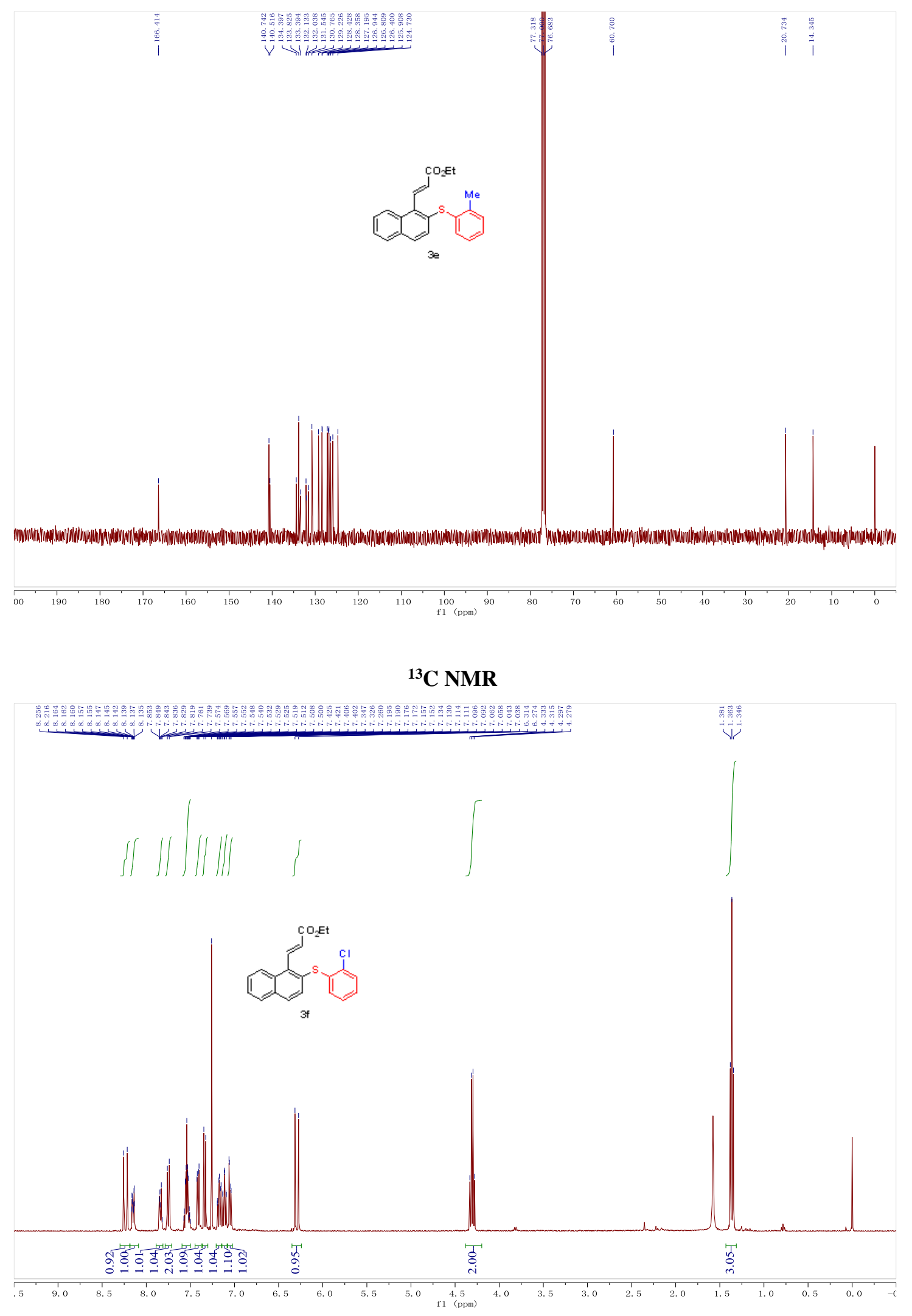

${ }^{1}$ H NMR 

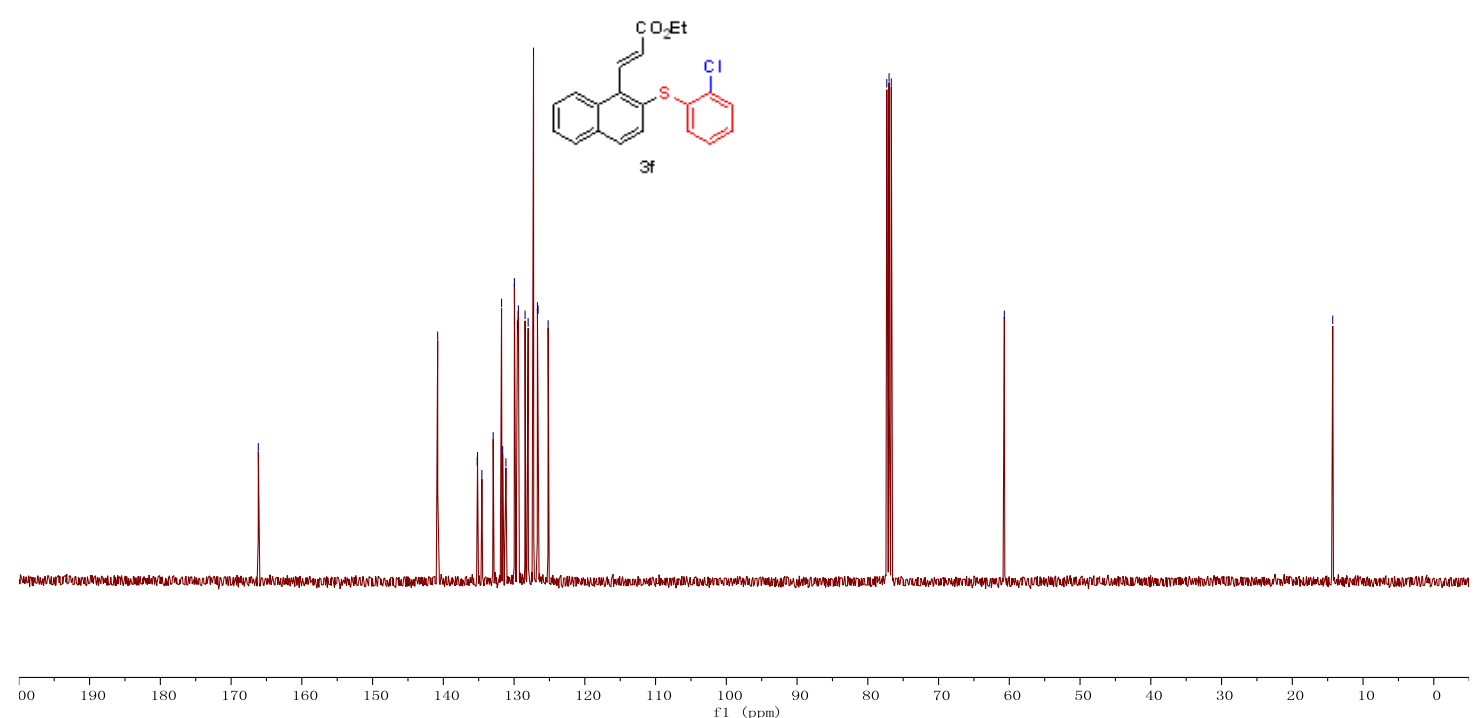

${ }^{13}$ C NMR

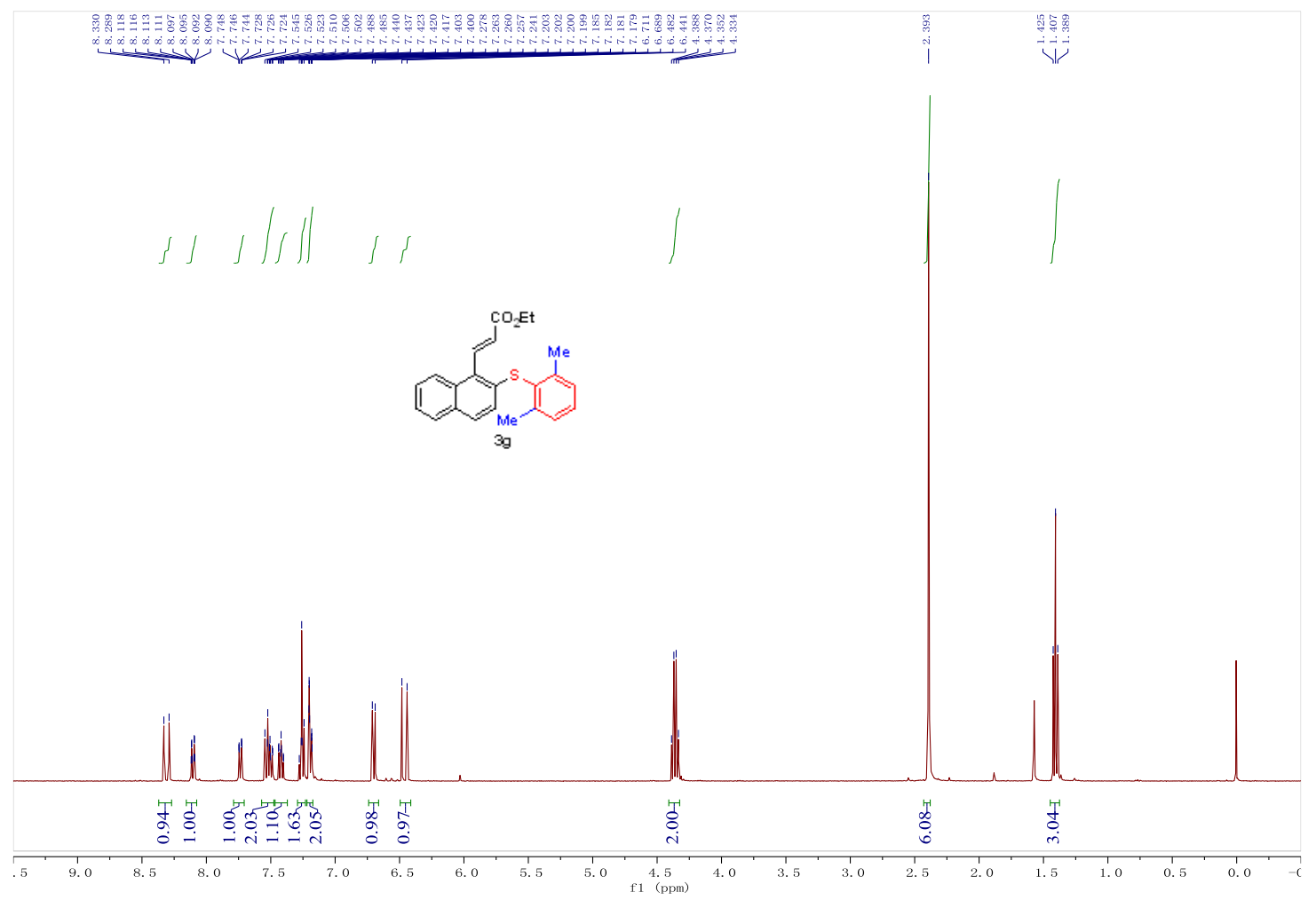

${ }^{1}$ H NMR 


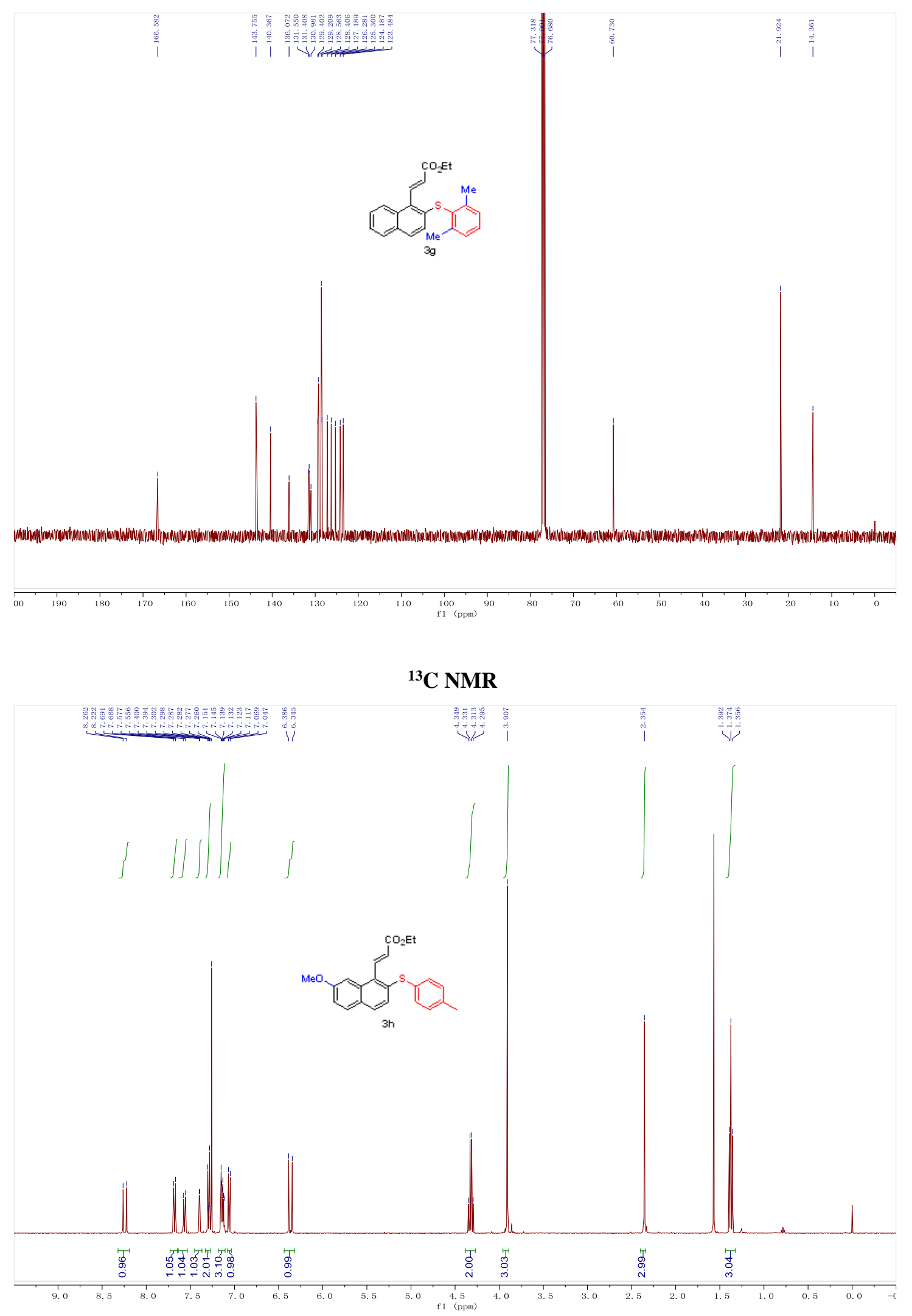

${ }^{1}$ H NMR 


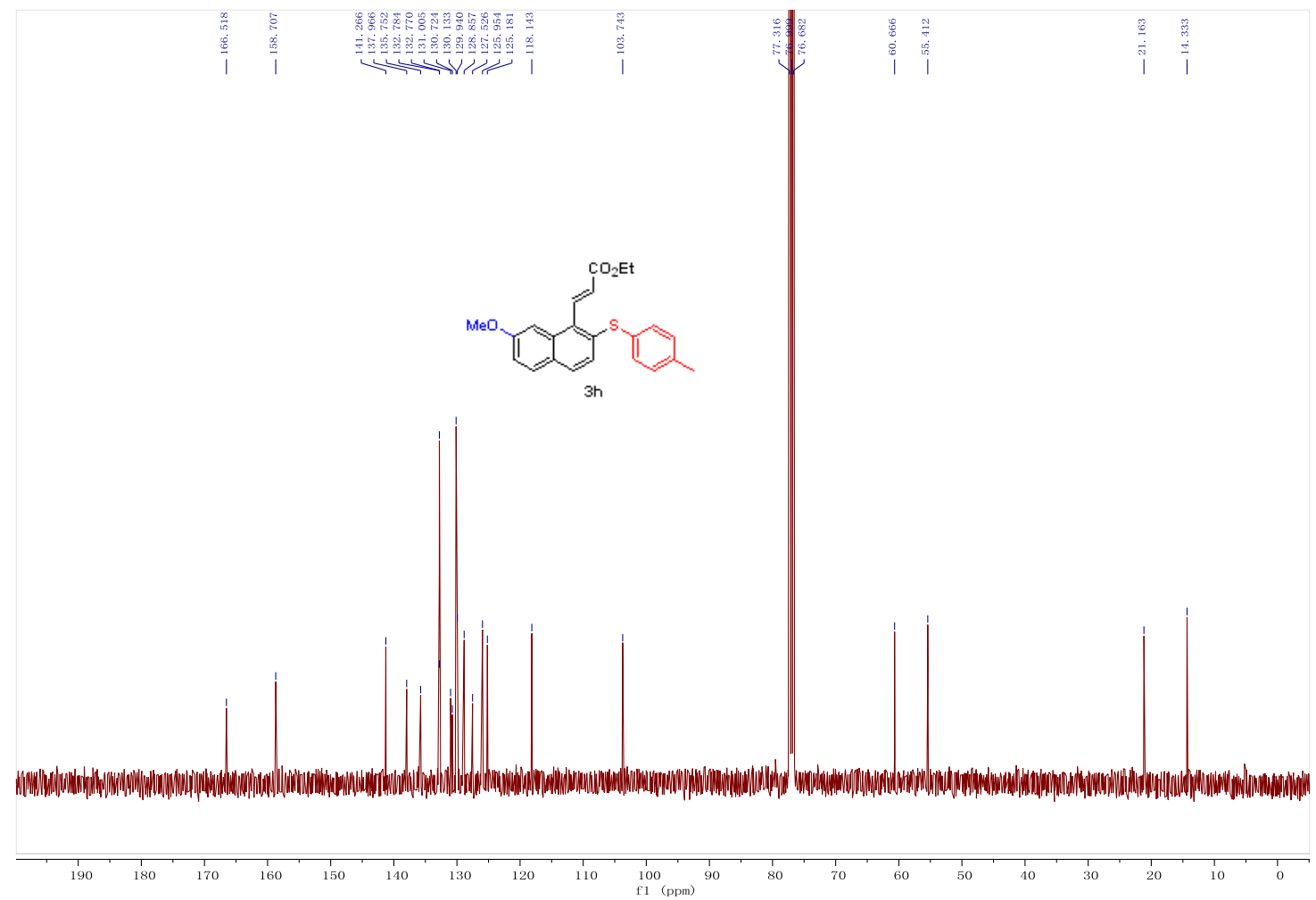

${ }^{13}$ C NMR

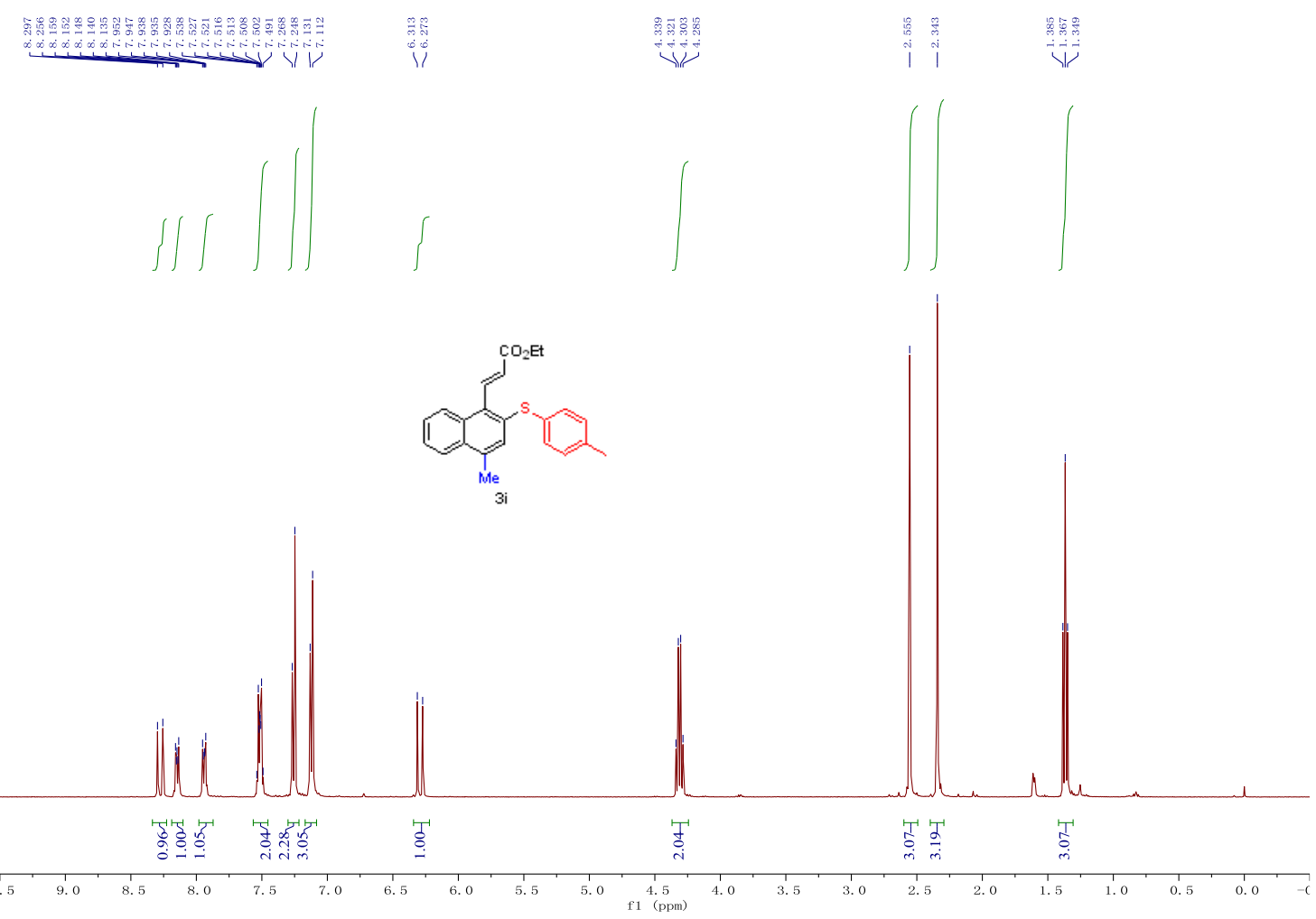

${ }^{1}$ H NMR

S27 

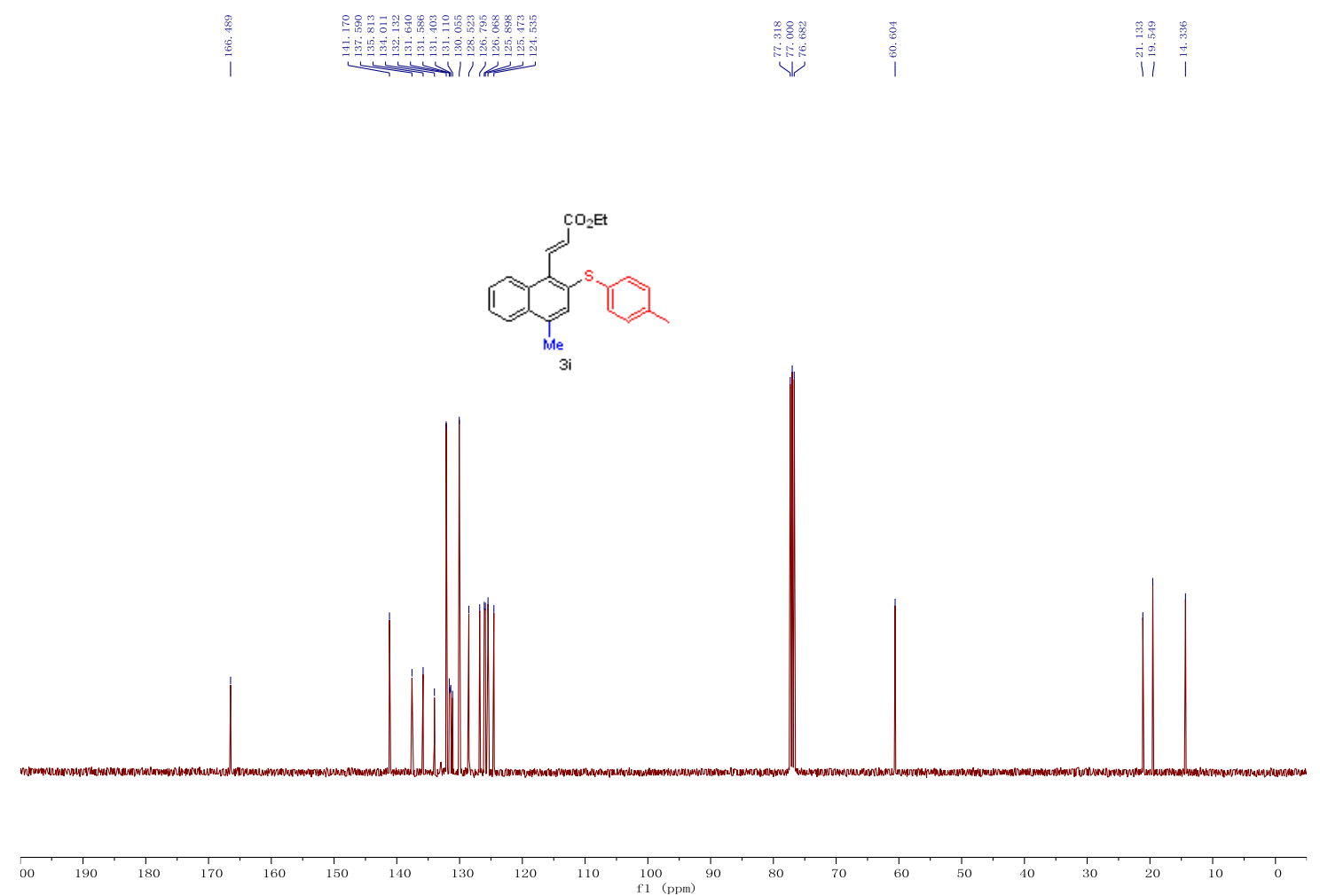

${ }^{13}$ C NMR

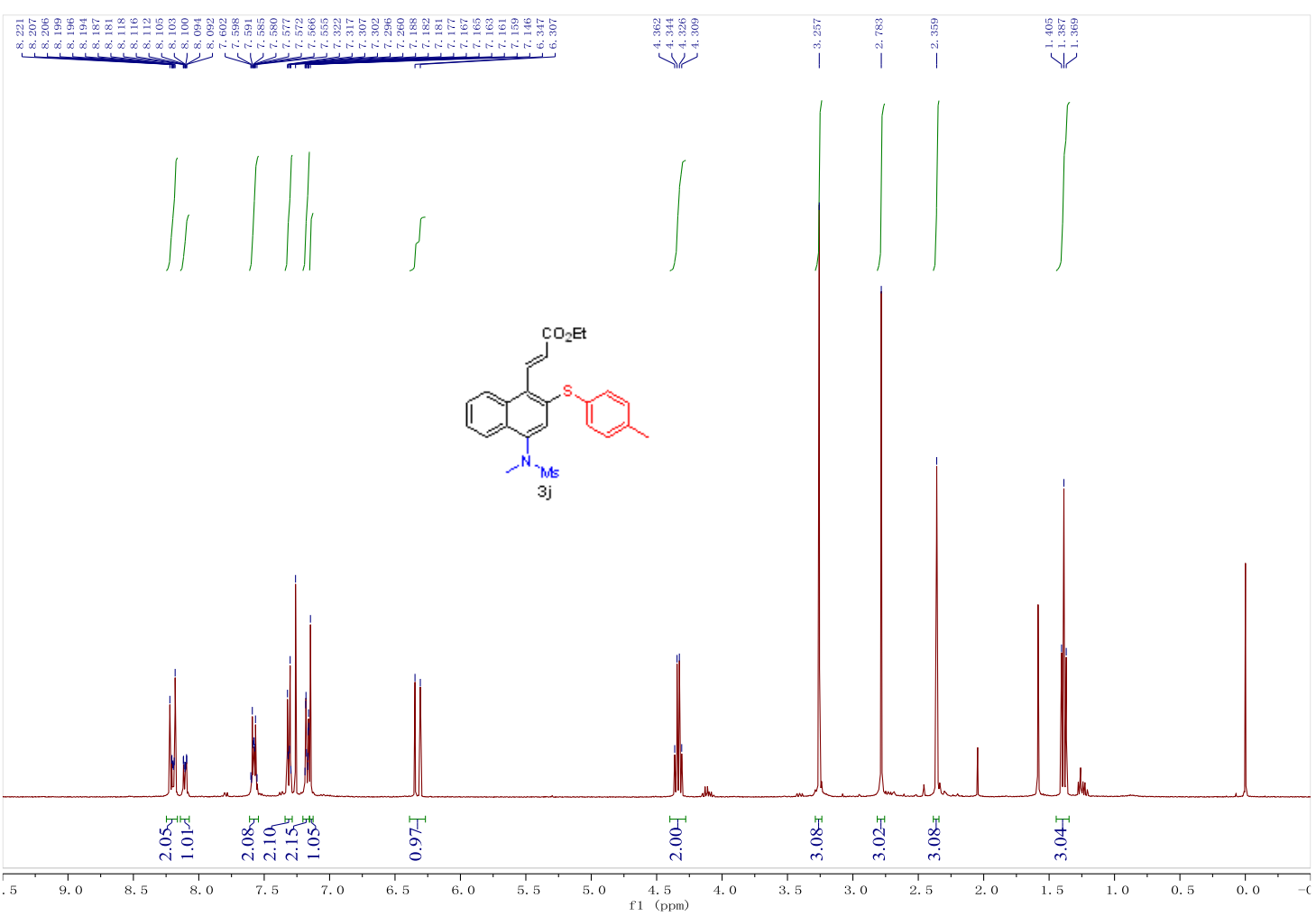

${ }^{1}$ H NMR 

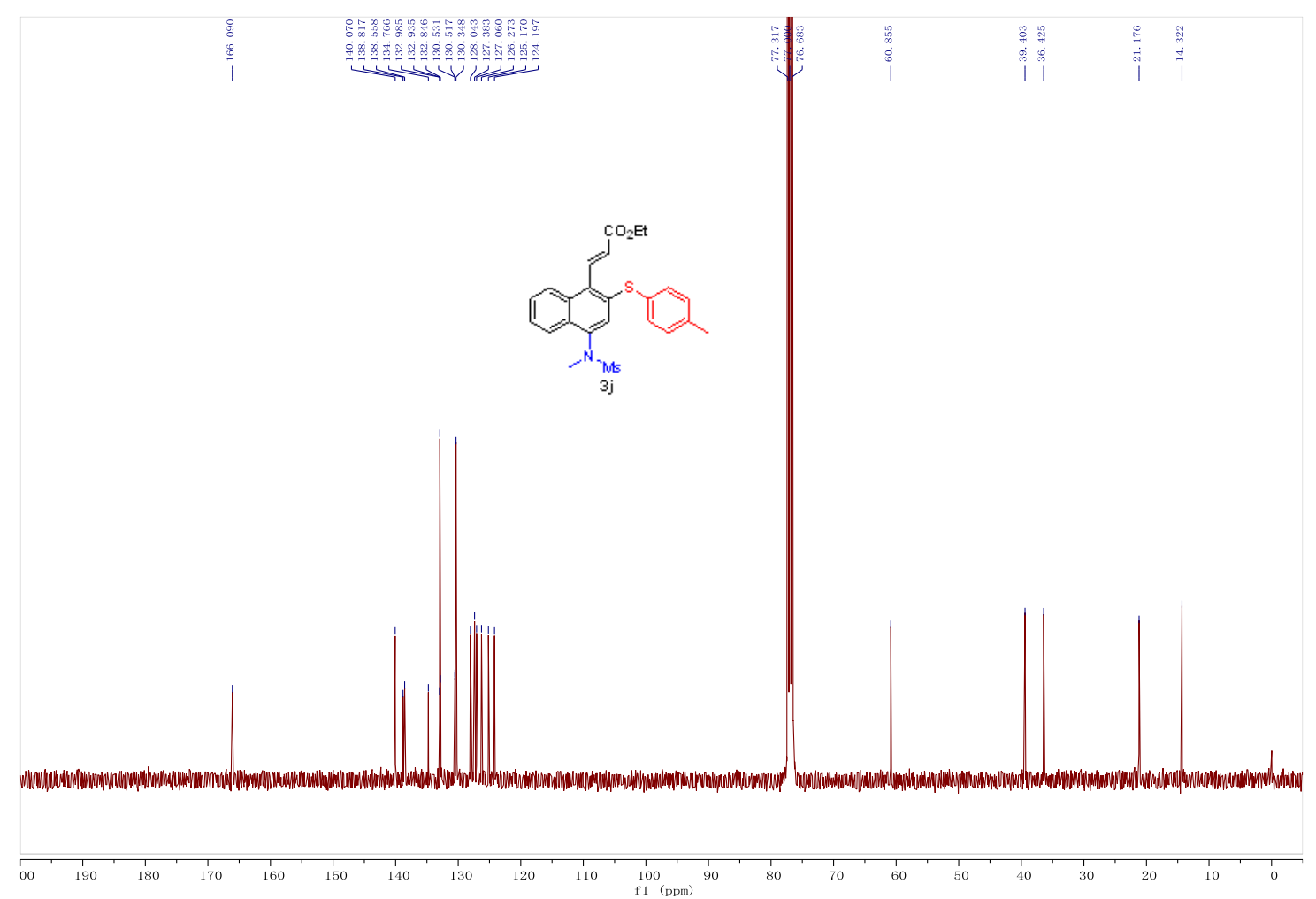

${ }^{13}$ C NMR

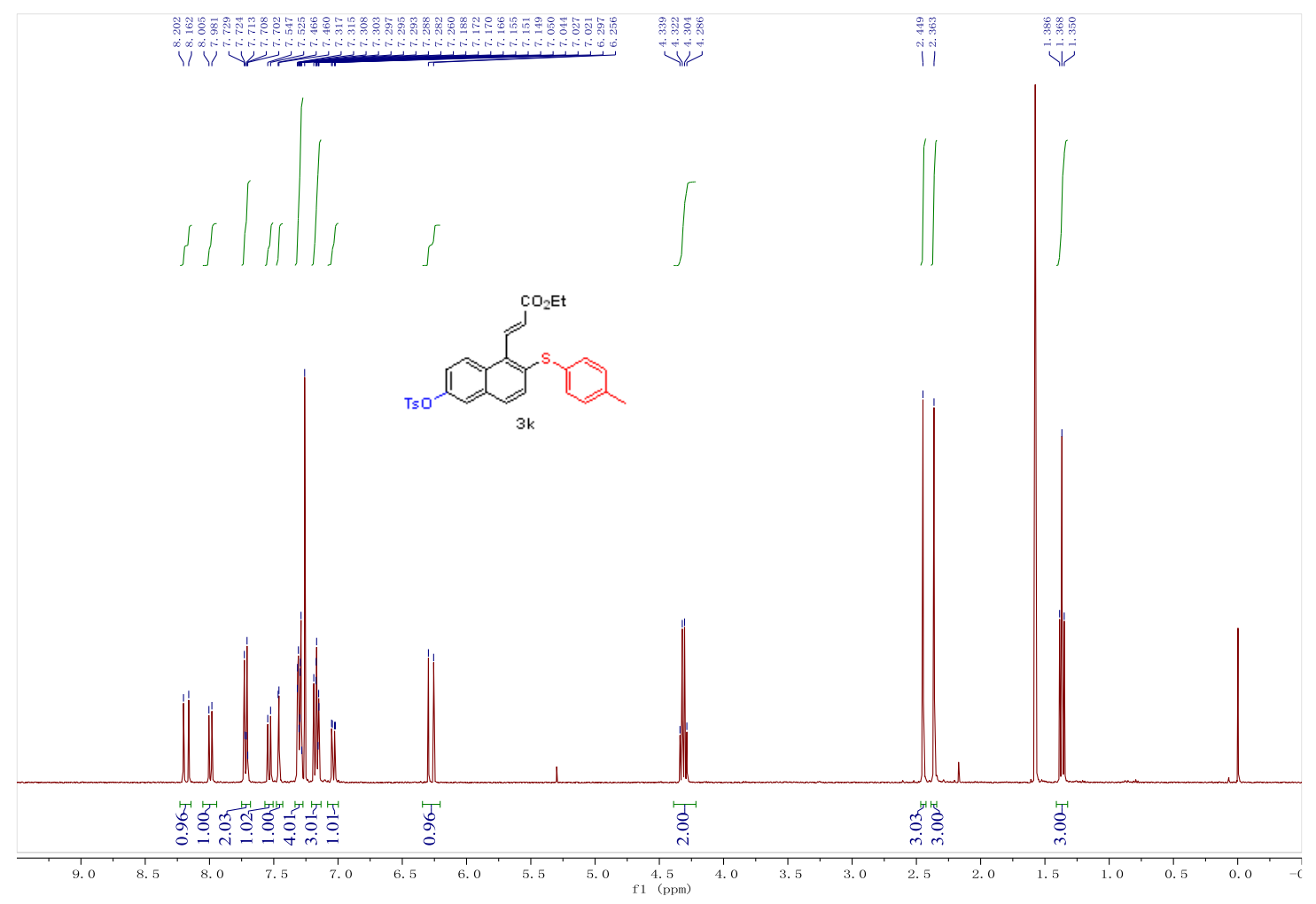

${ }^{1}$ H NMR 

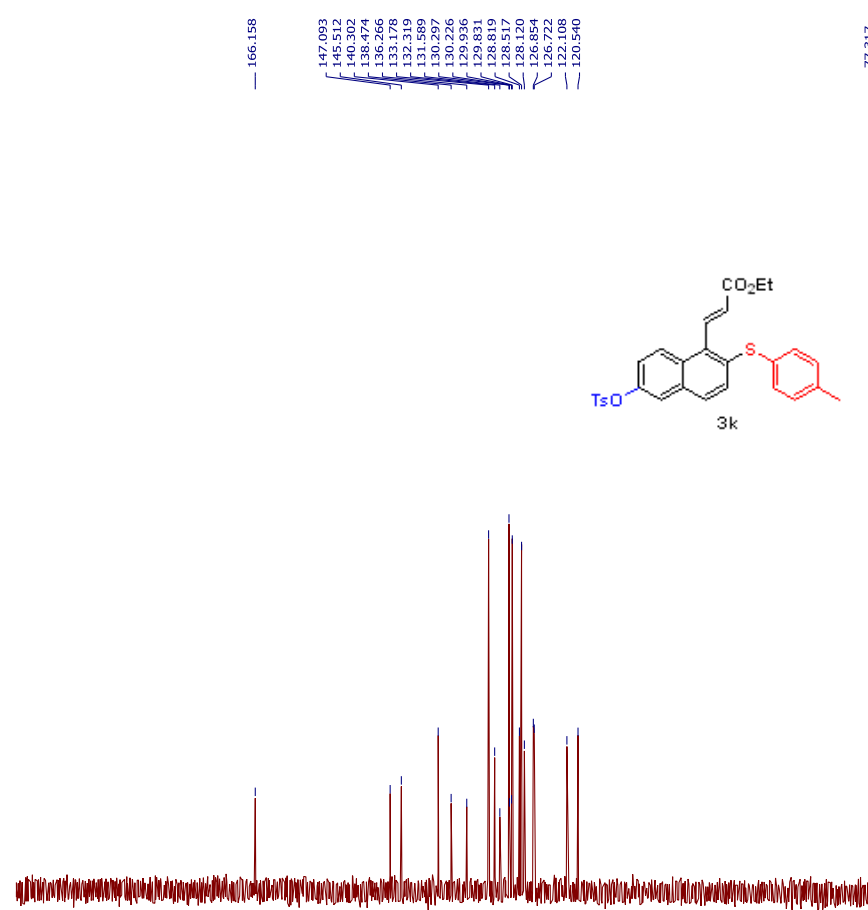

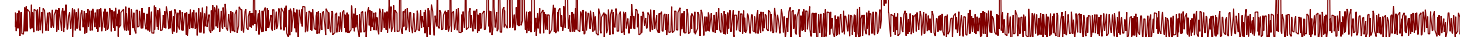

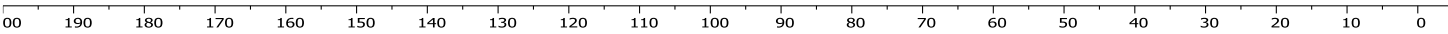

\section{${ }^{13}$ C NMR}

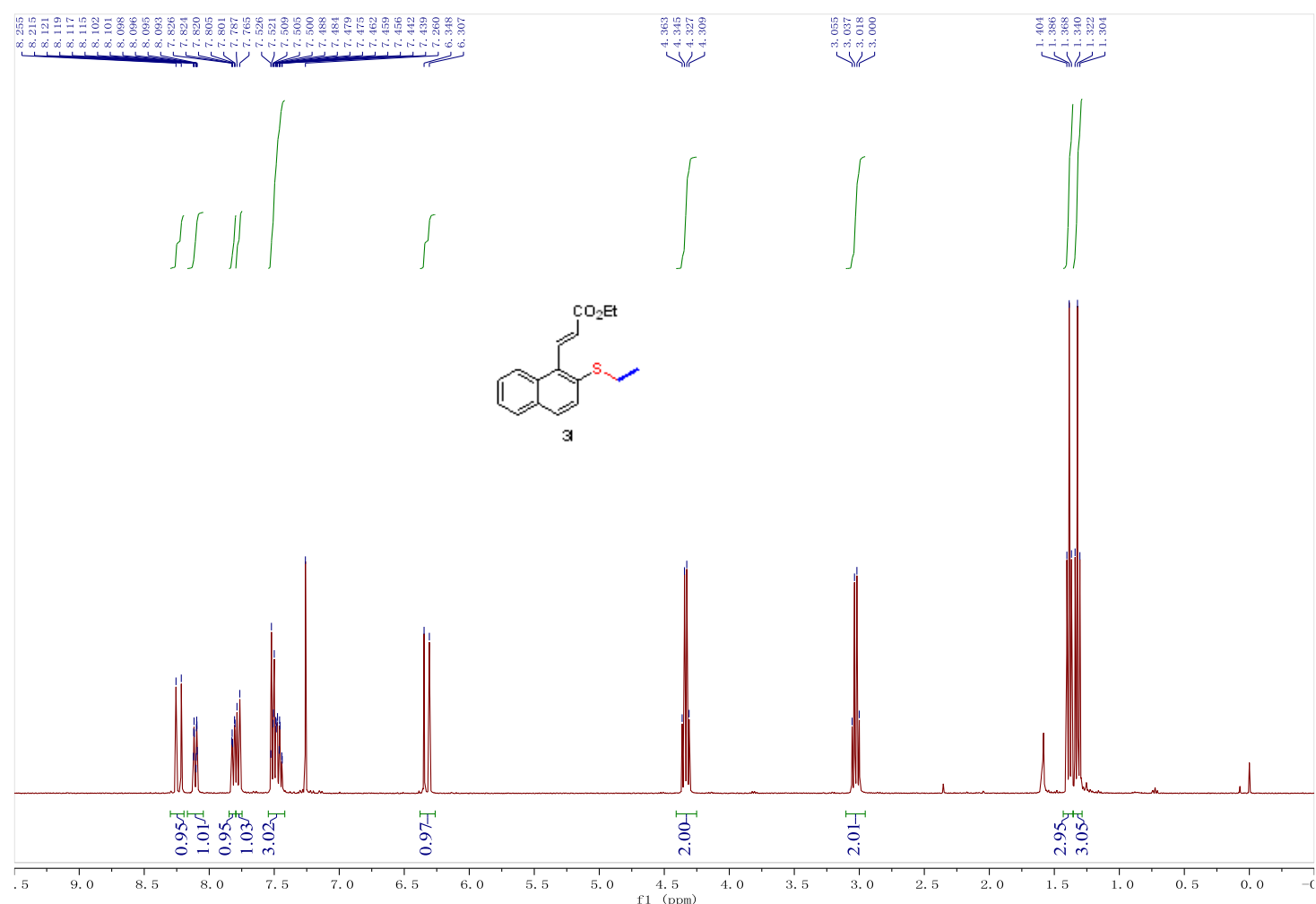

${ }^{1}$ H NMR 

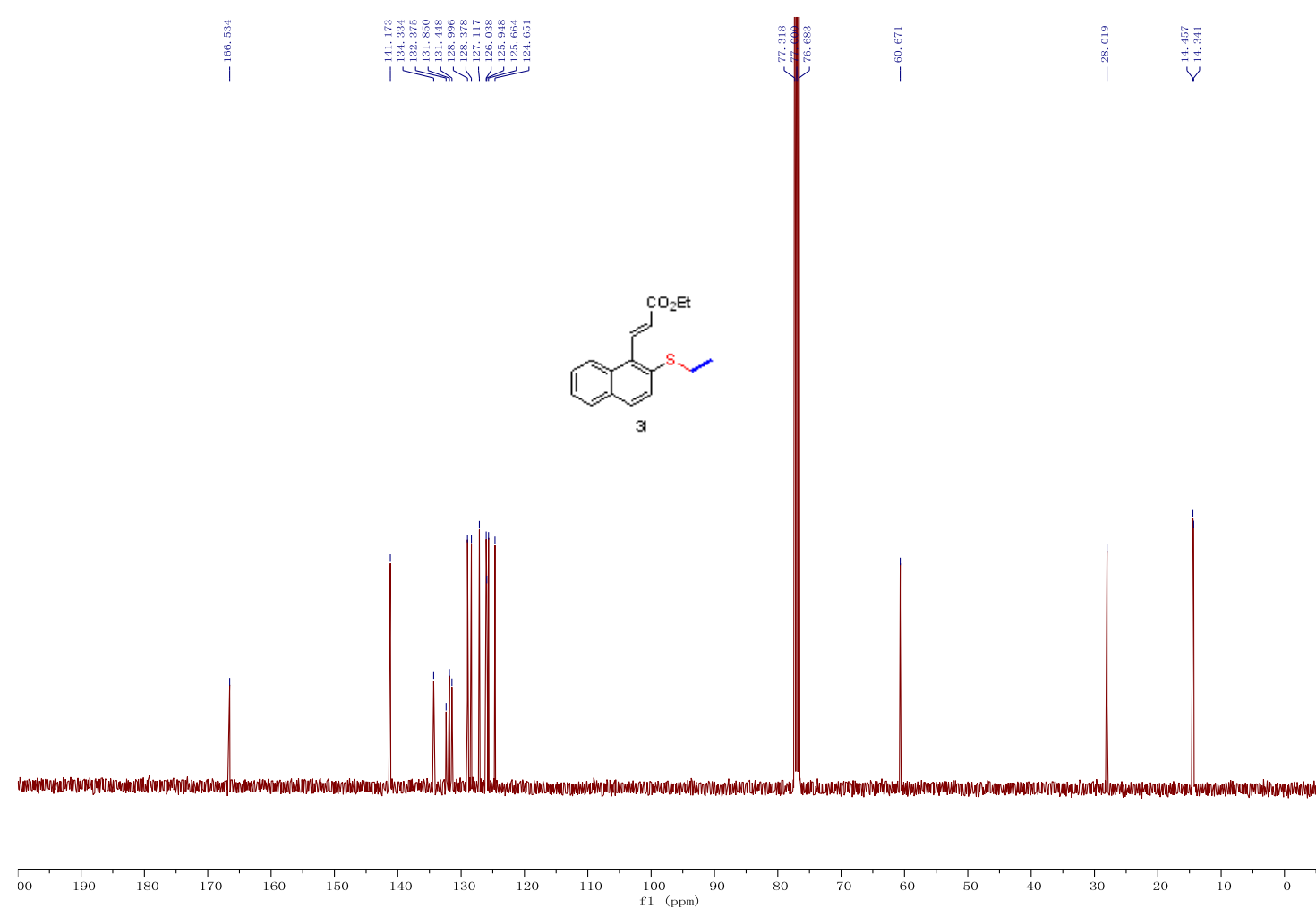

${ }^{13}$ C NMR

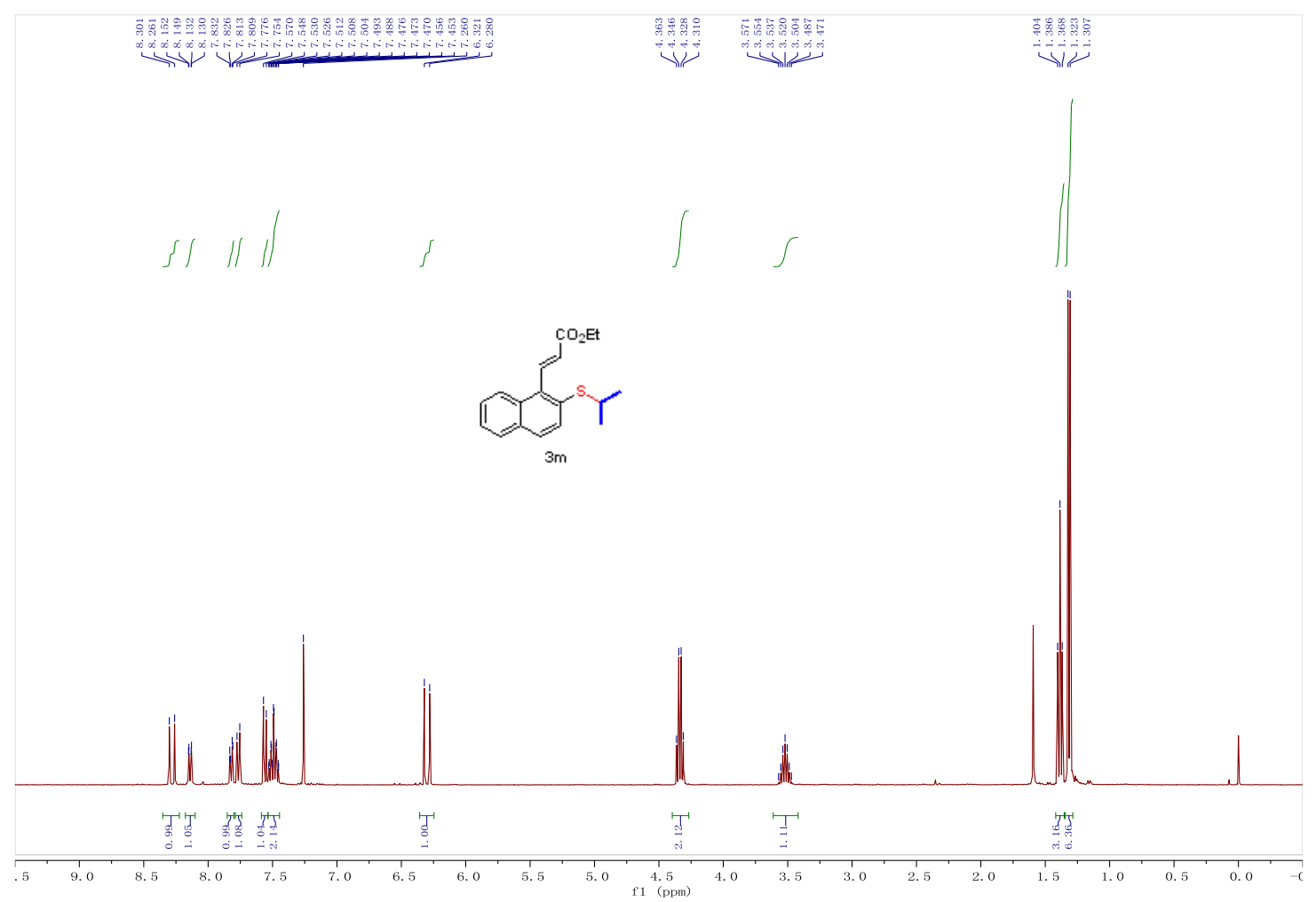

${ }^{1}$ H NMR 


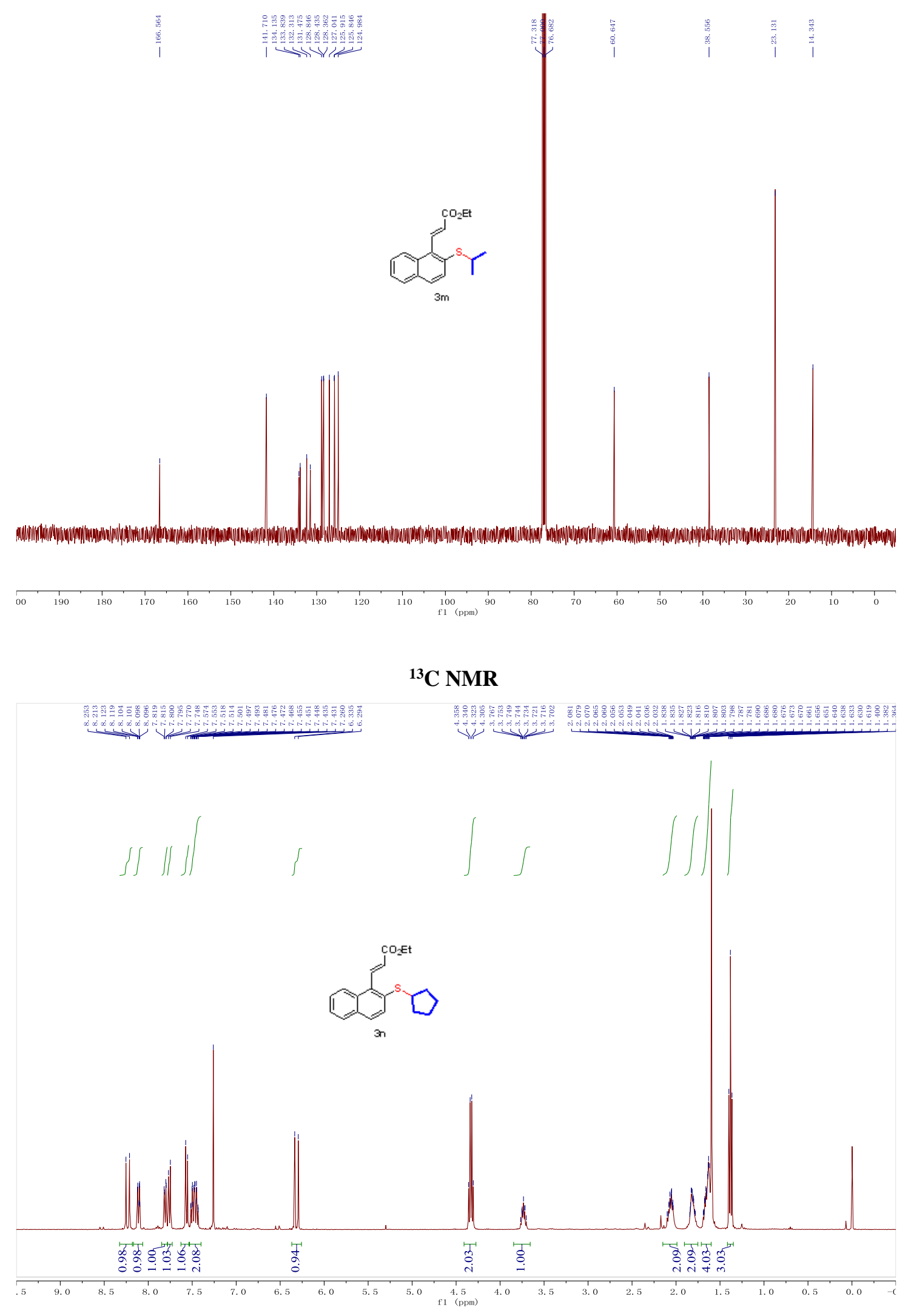

${ }^{1}$ H NMR 

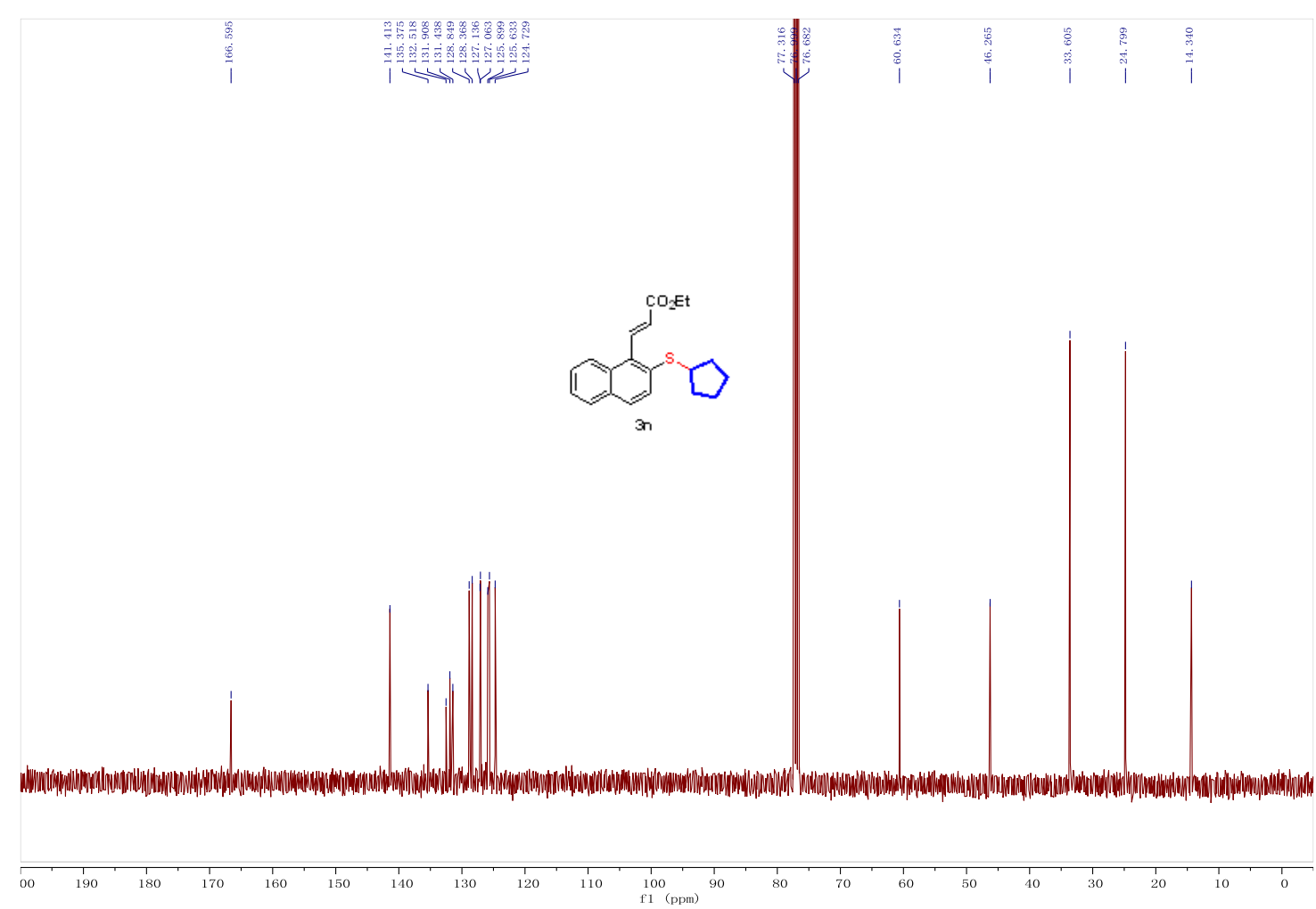

${ }^{13}$ C NMR

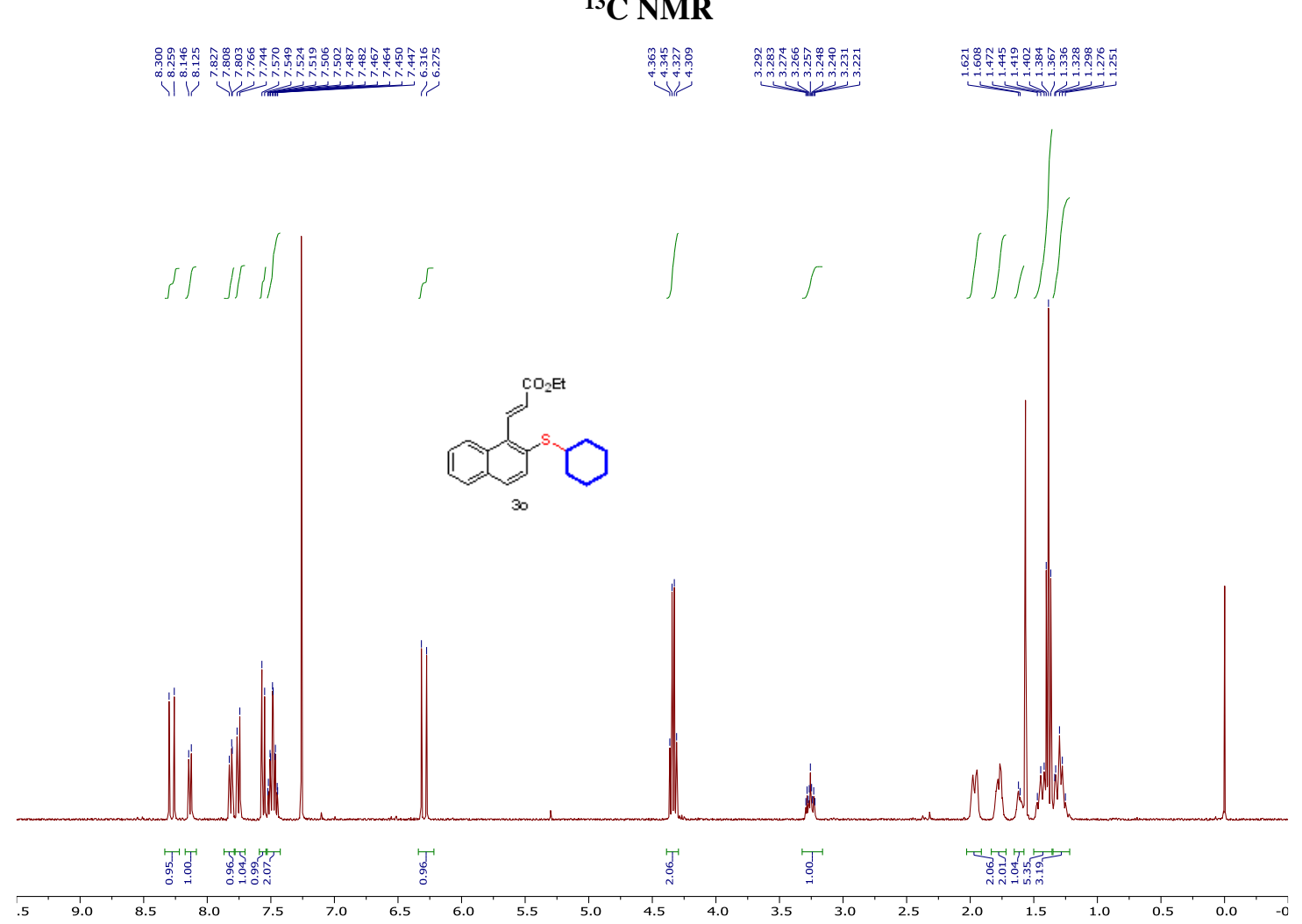

${ }^{1}$ H NMR 

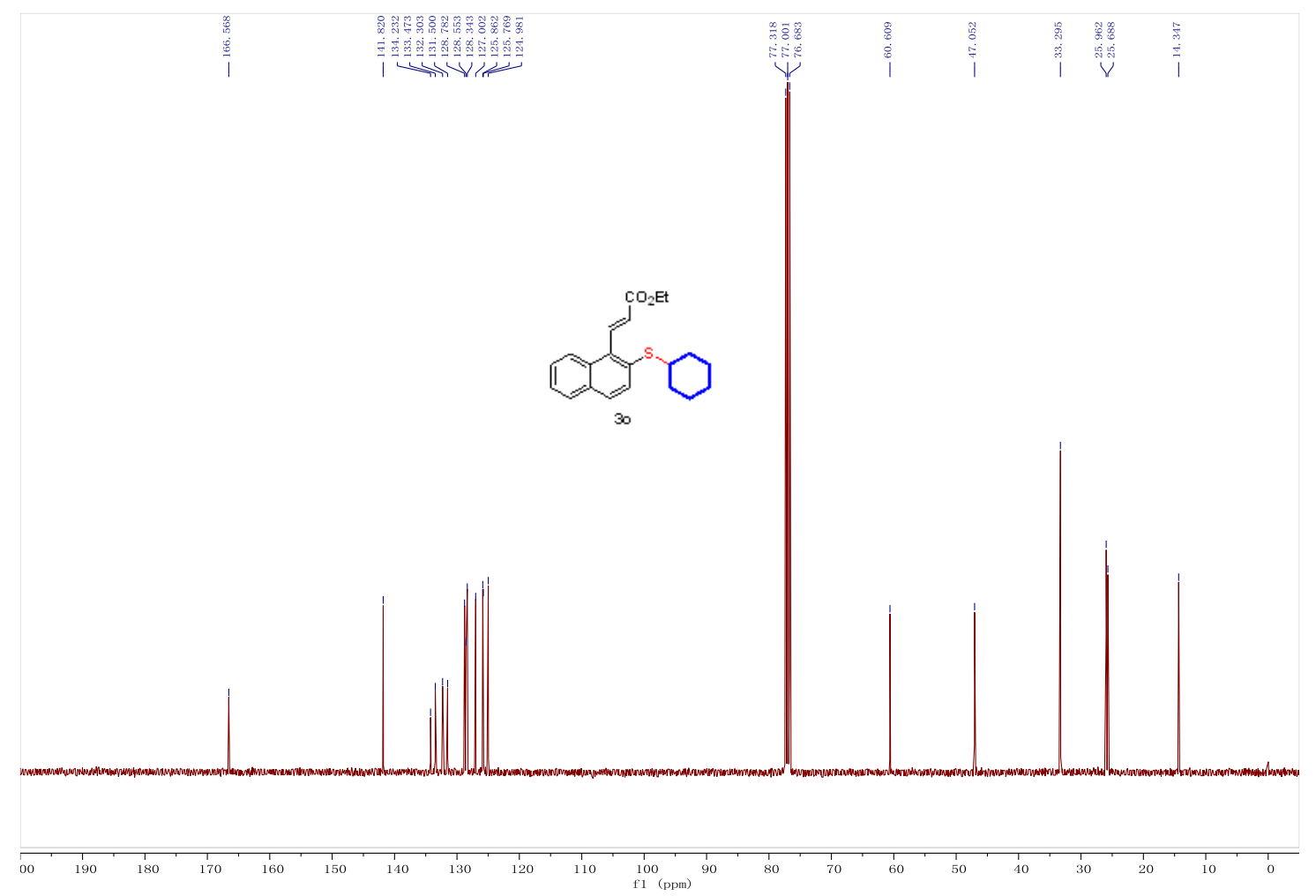

${ }^{13}$ C NMR

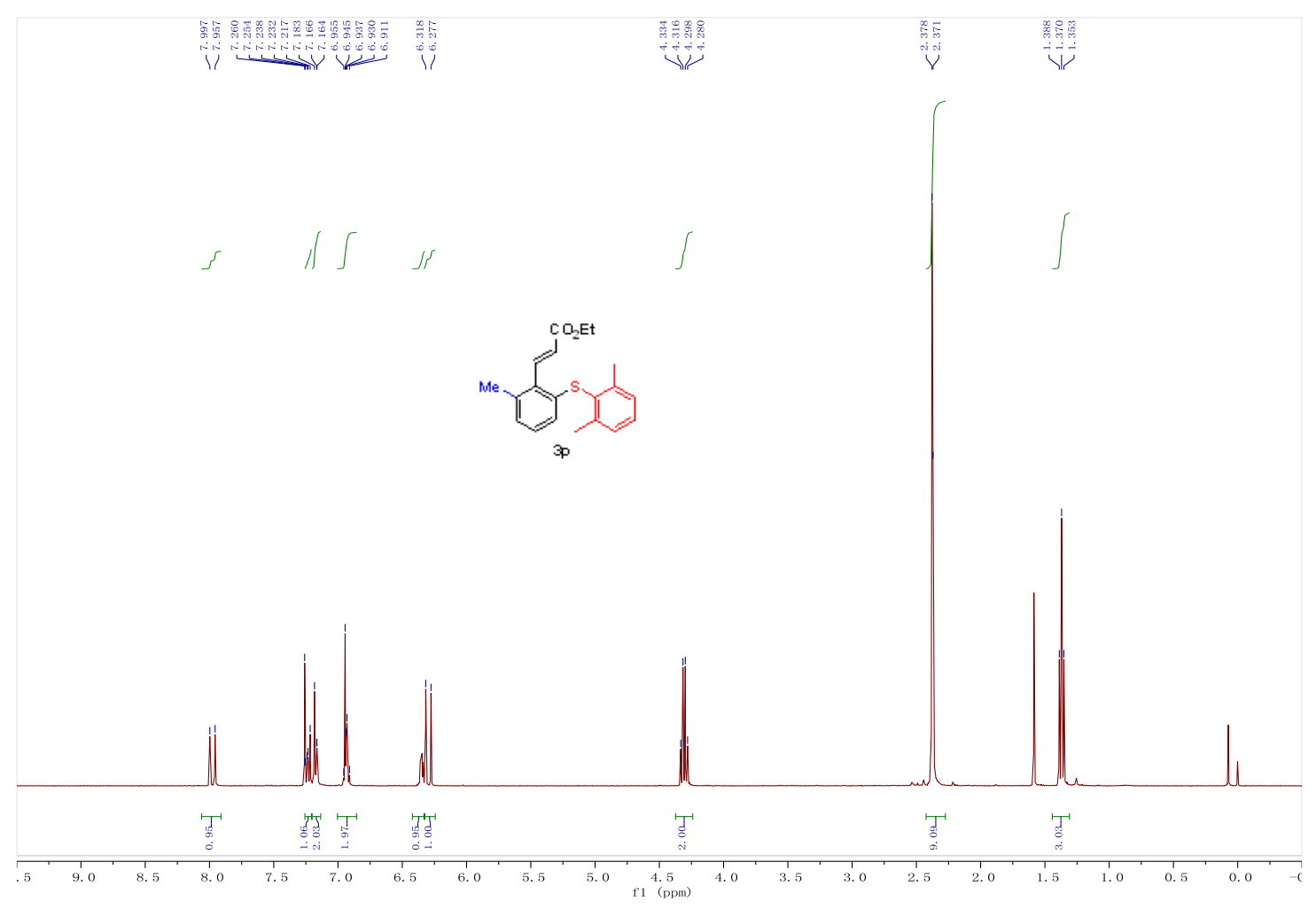

${ }^{1}$ H NMR 

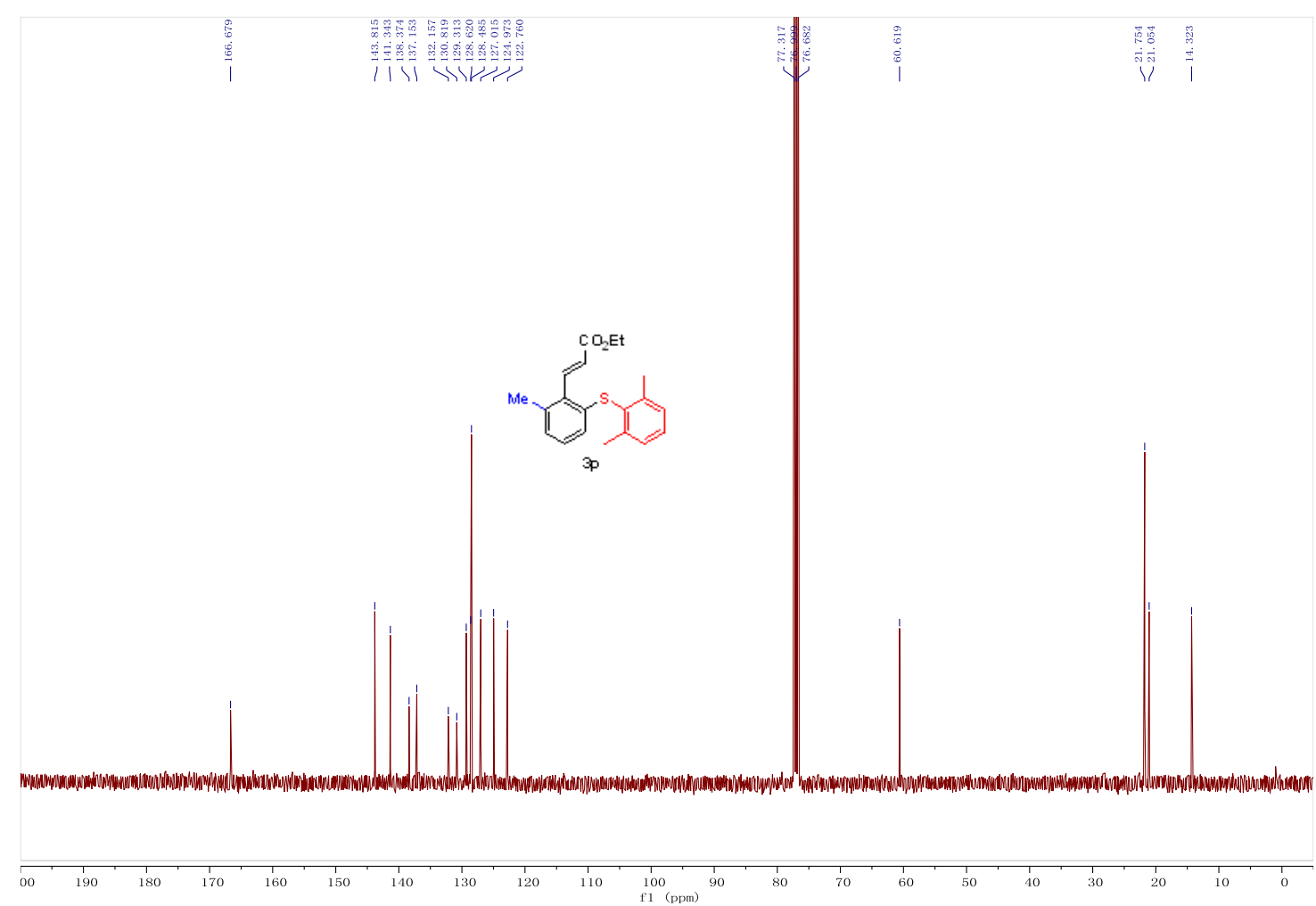

${ }^{13}$ C NMR

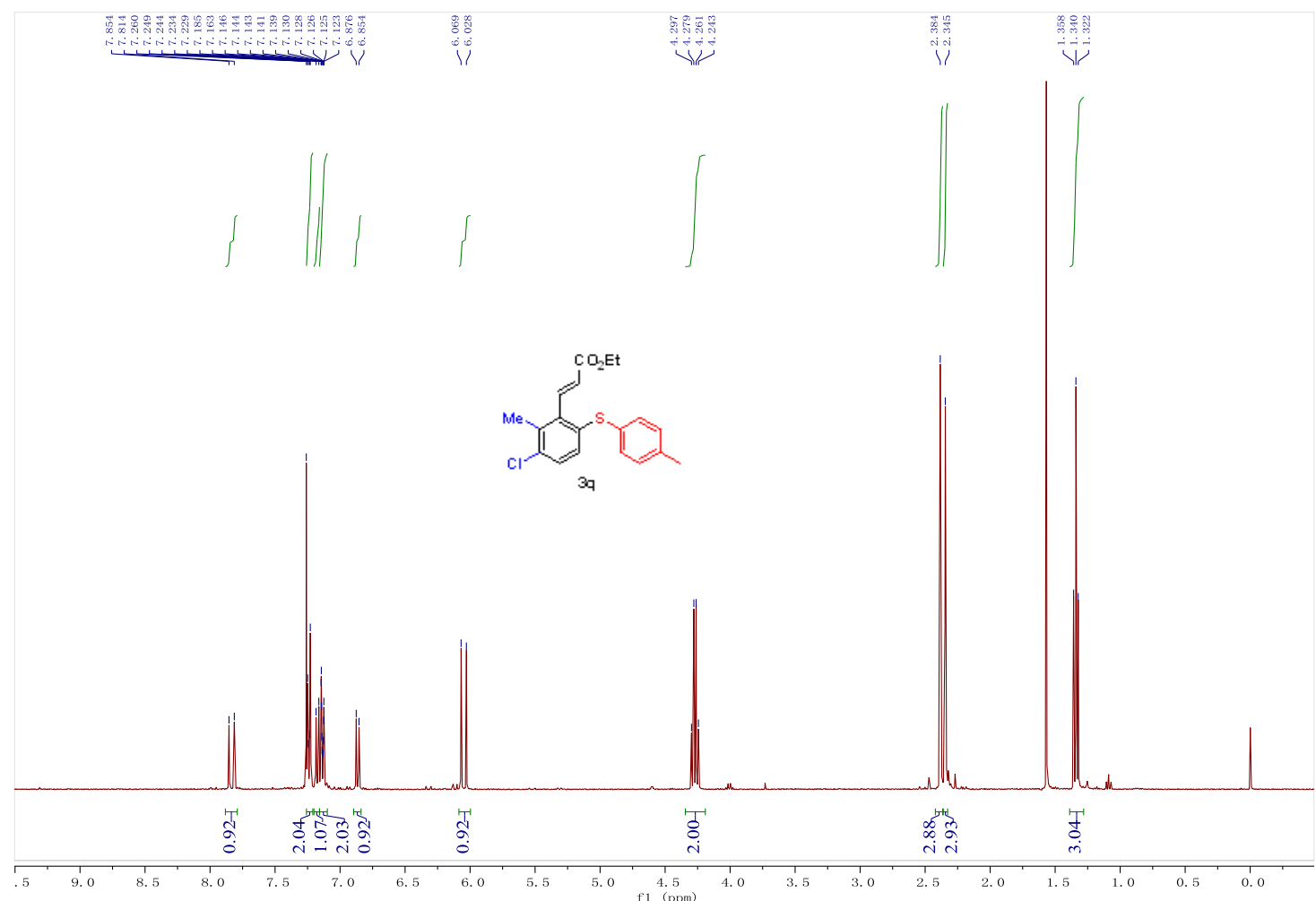

${ }^{1}$ H NMR 

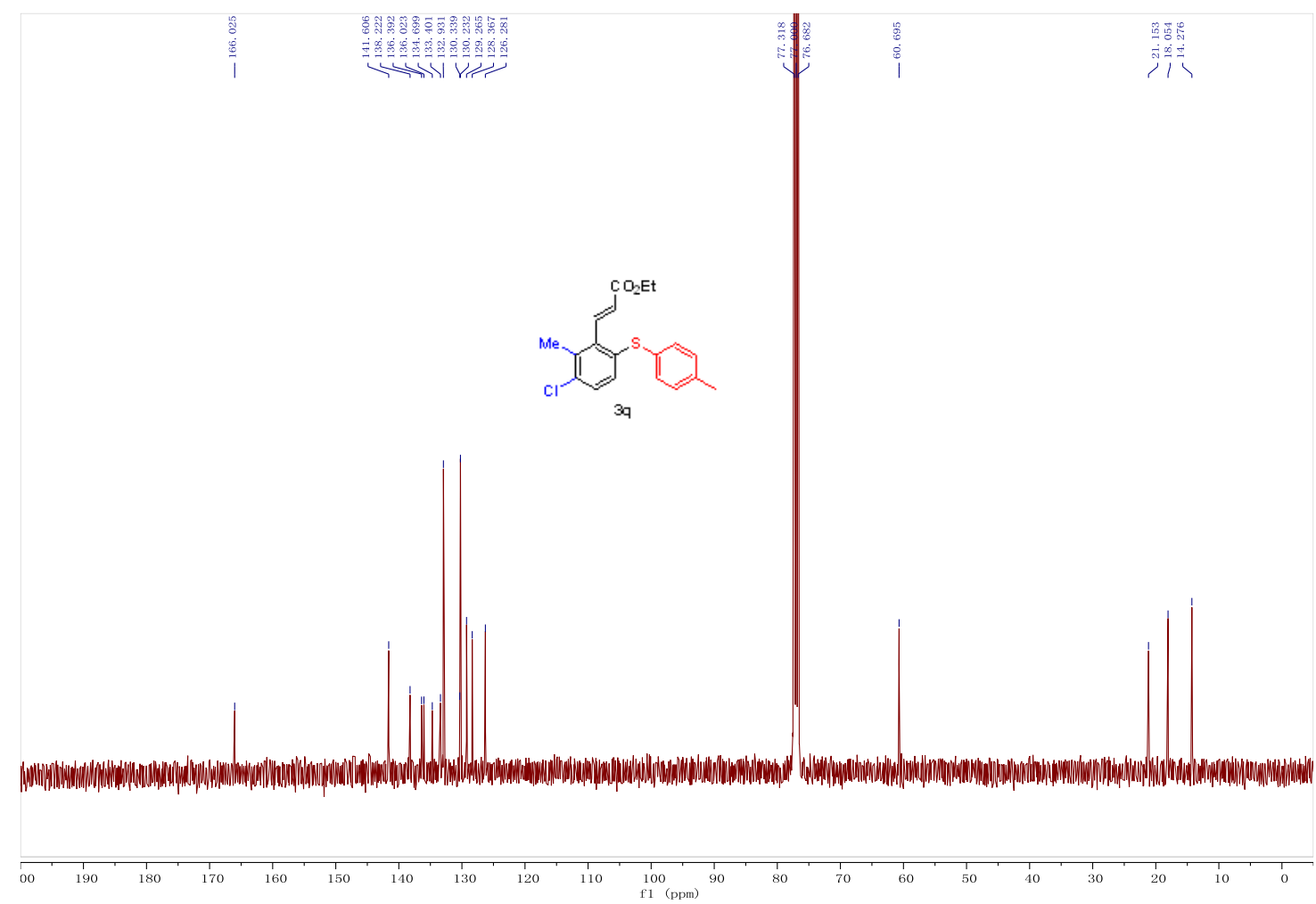

${ }^{13}$ C NMR

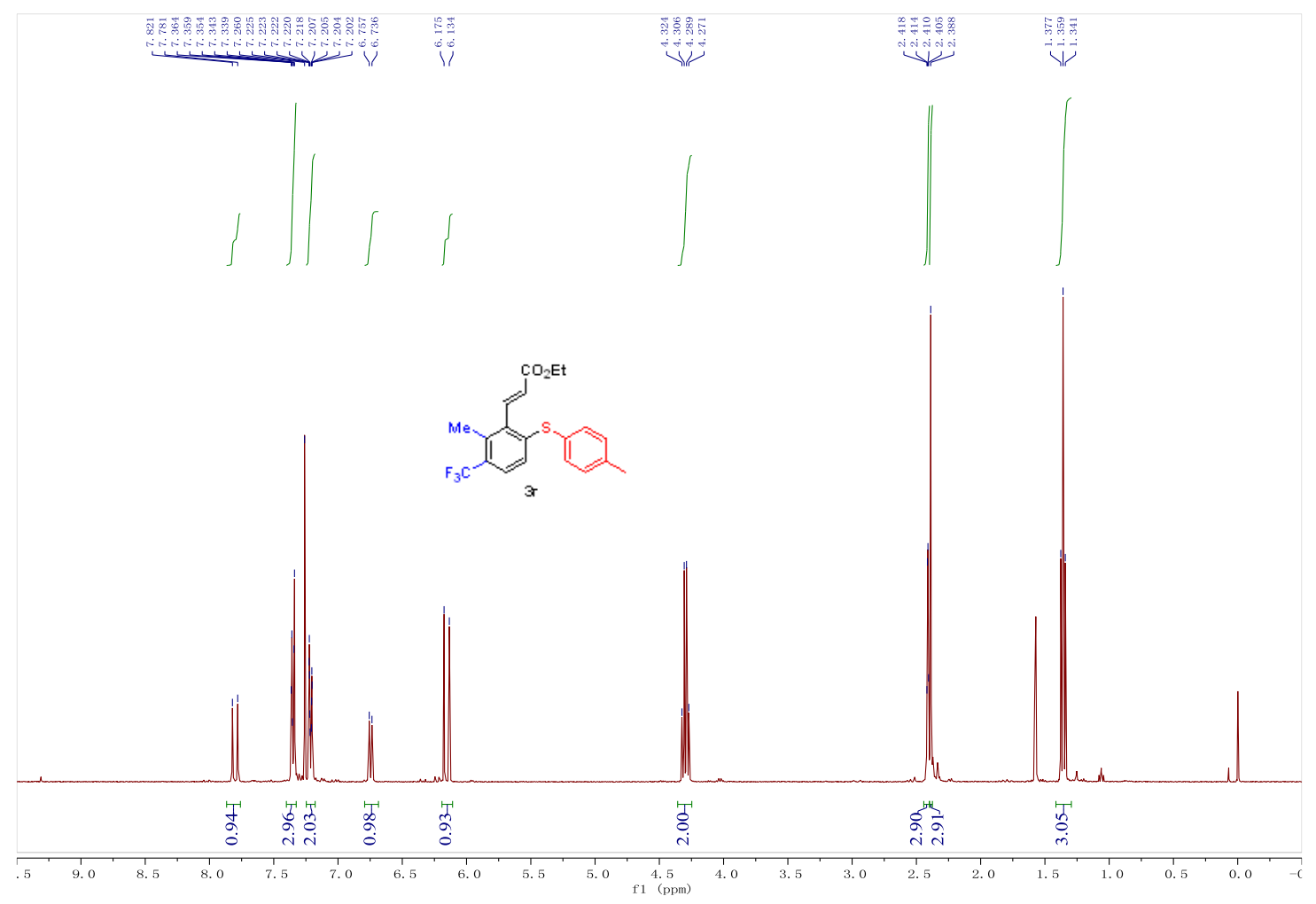

${ }^{1}$ H NMR 


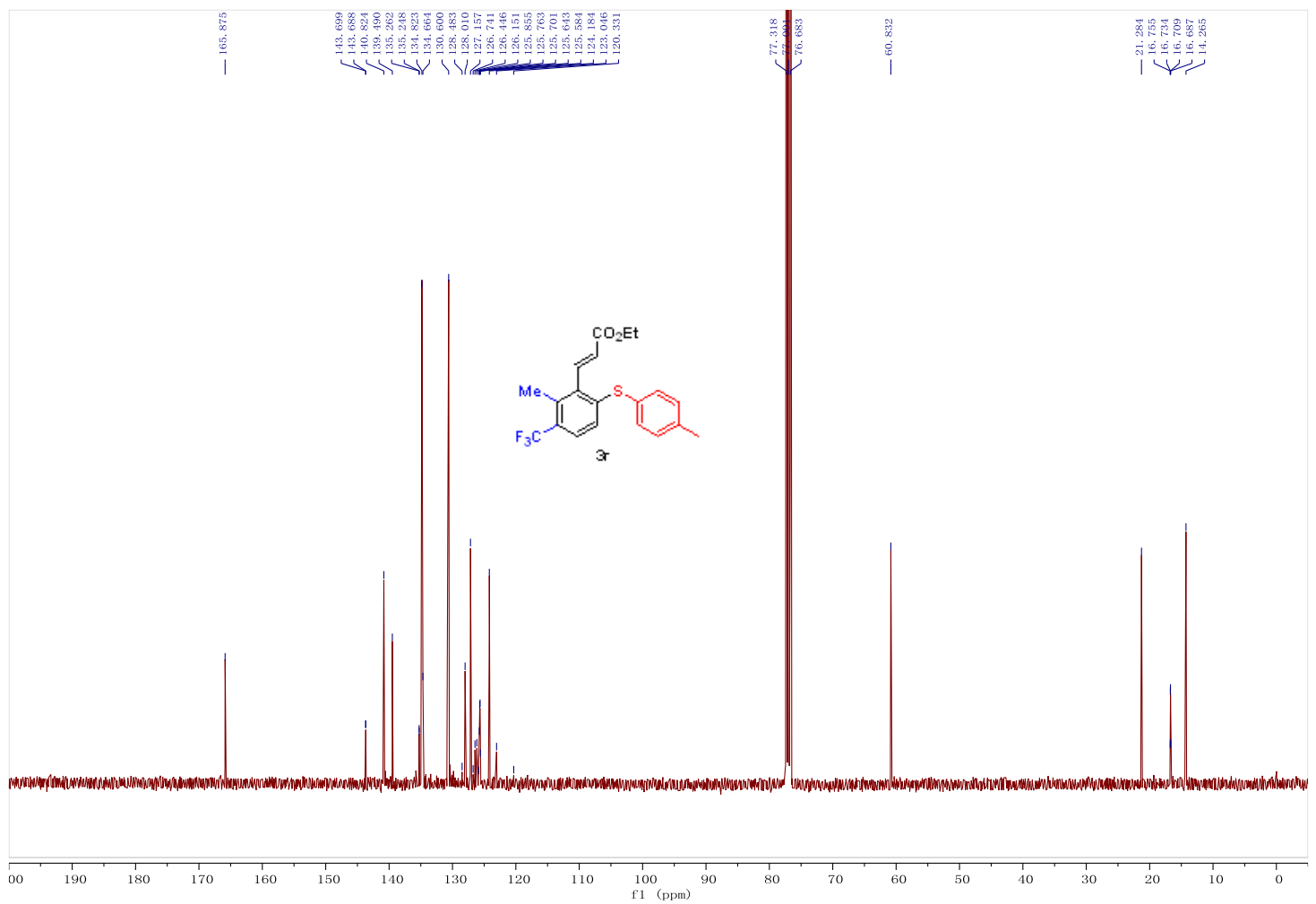

${ }^{13}$ C NMR

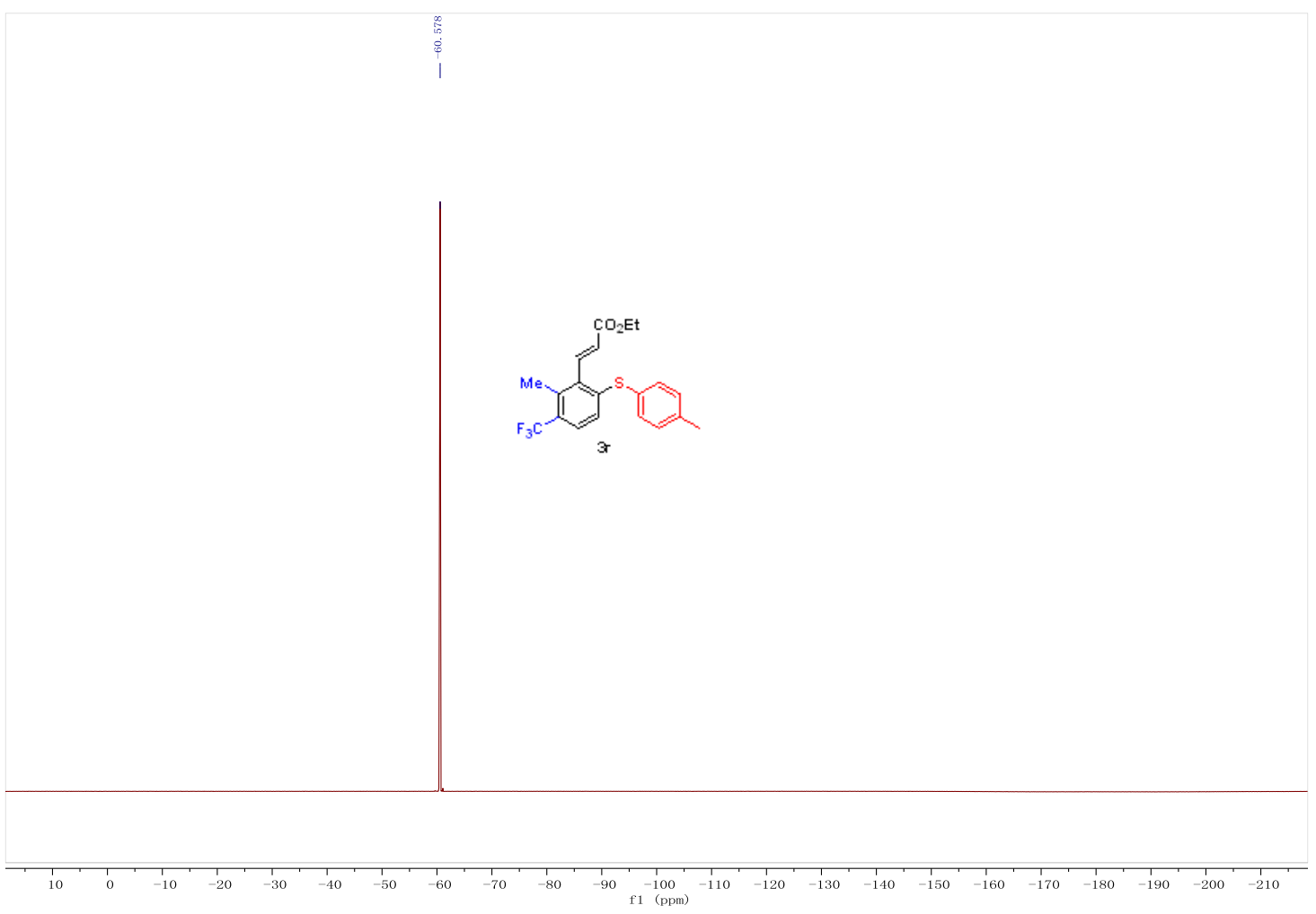

${ }^{19}$ F NMR

S37 


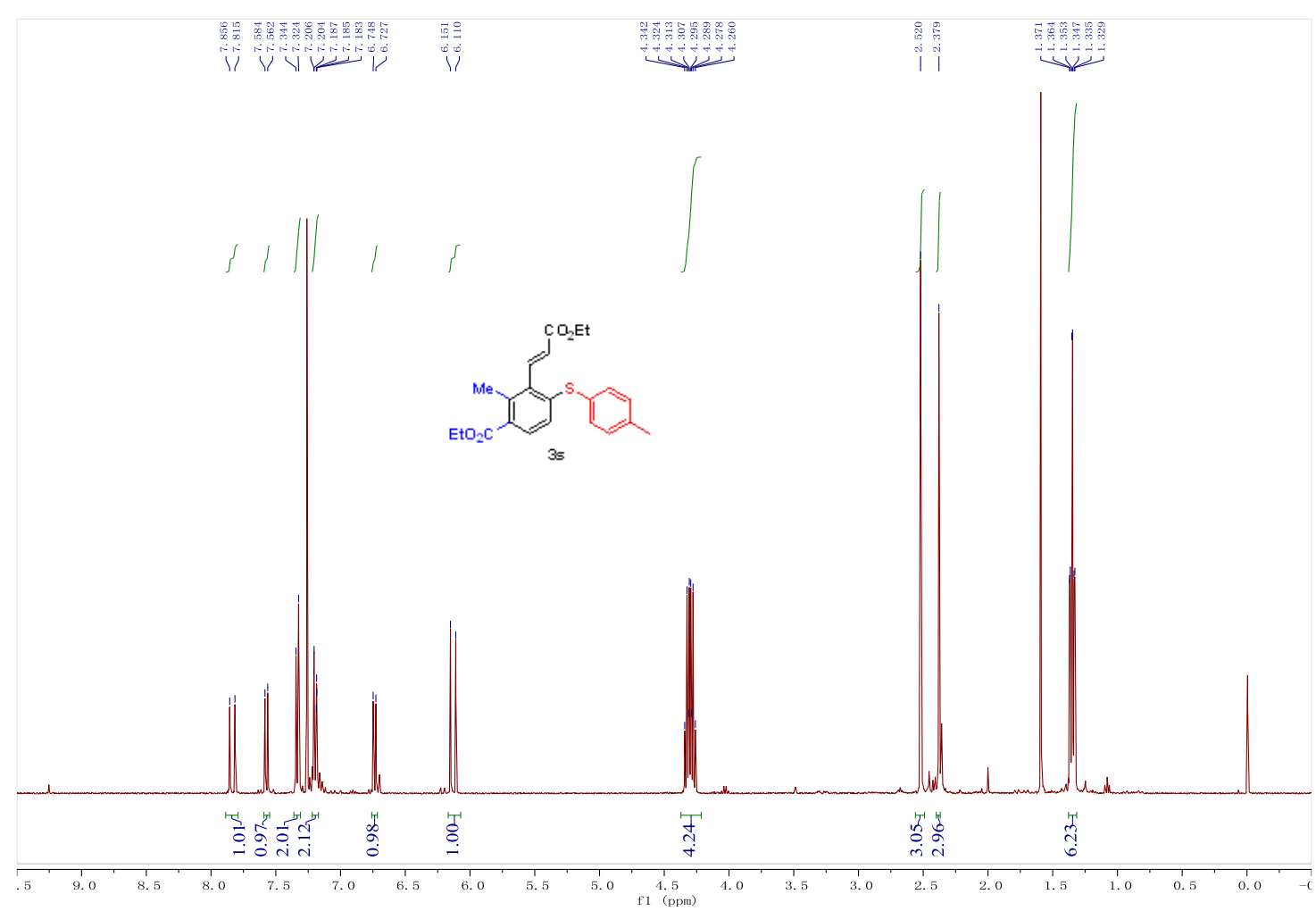

${ }^{1}$ H NMR
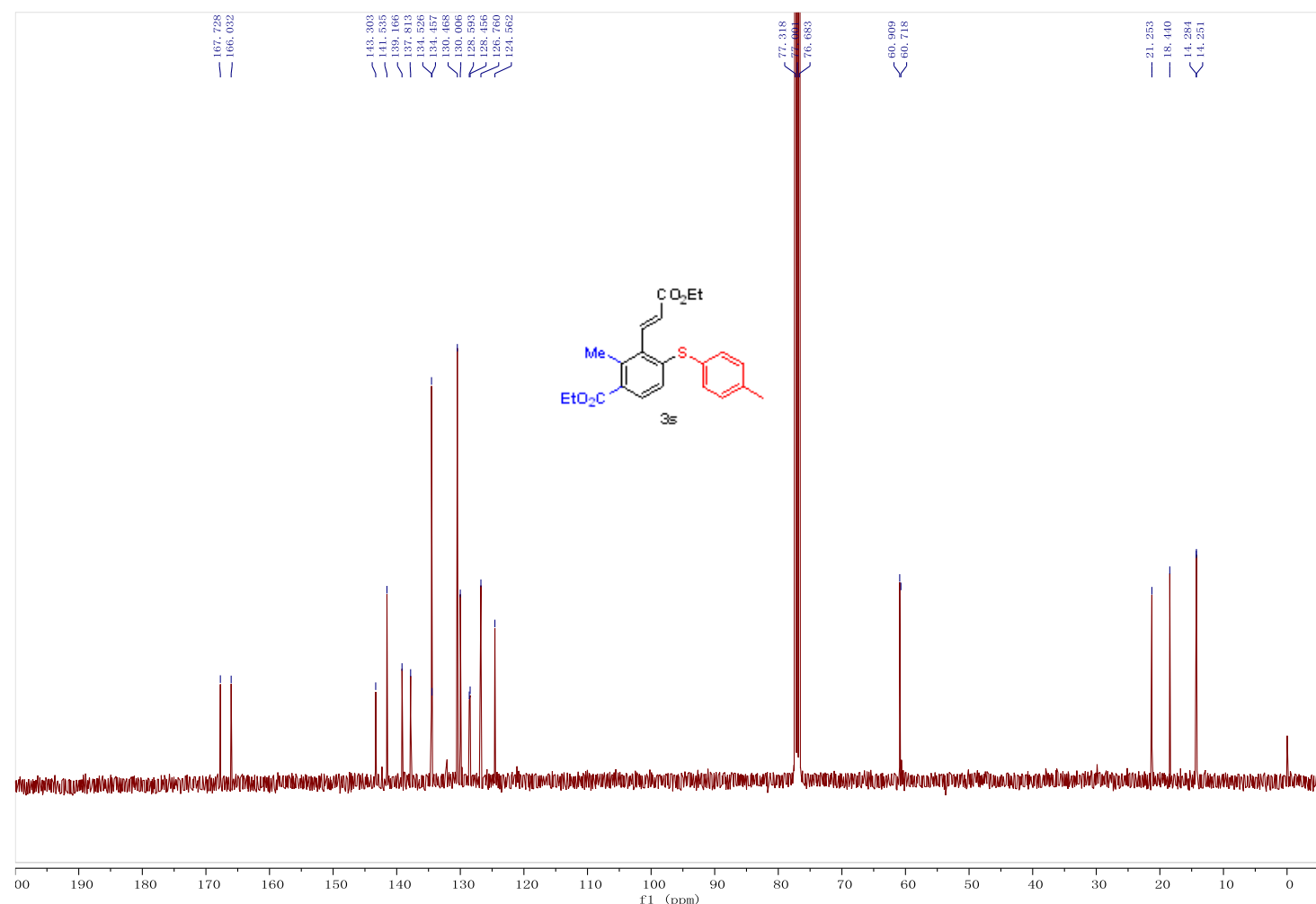

${ }^{13}$ C NMR 


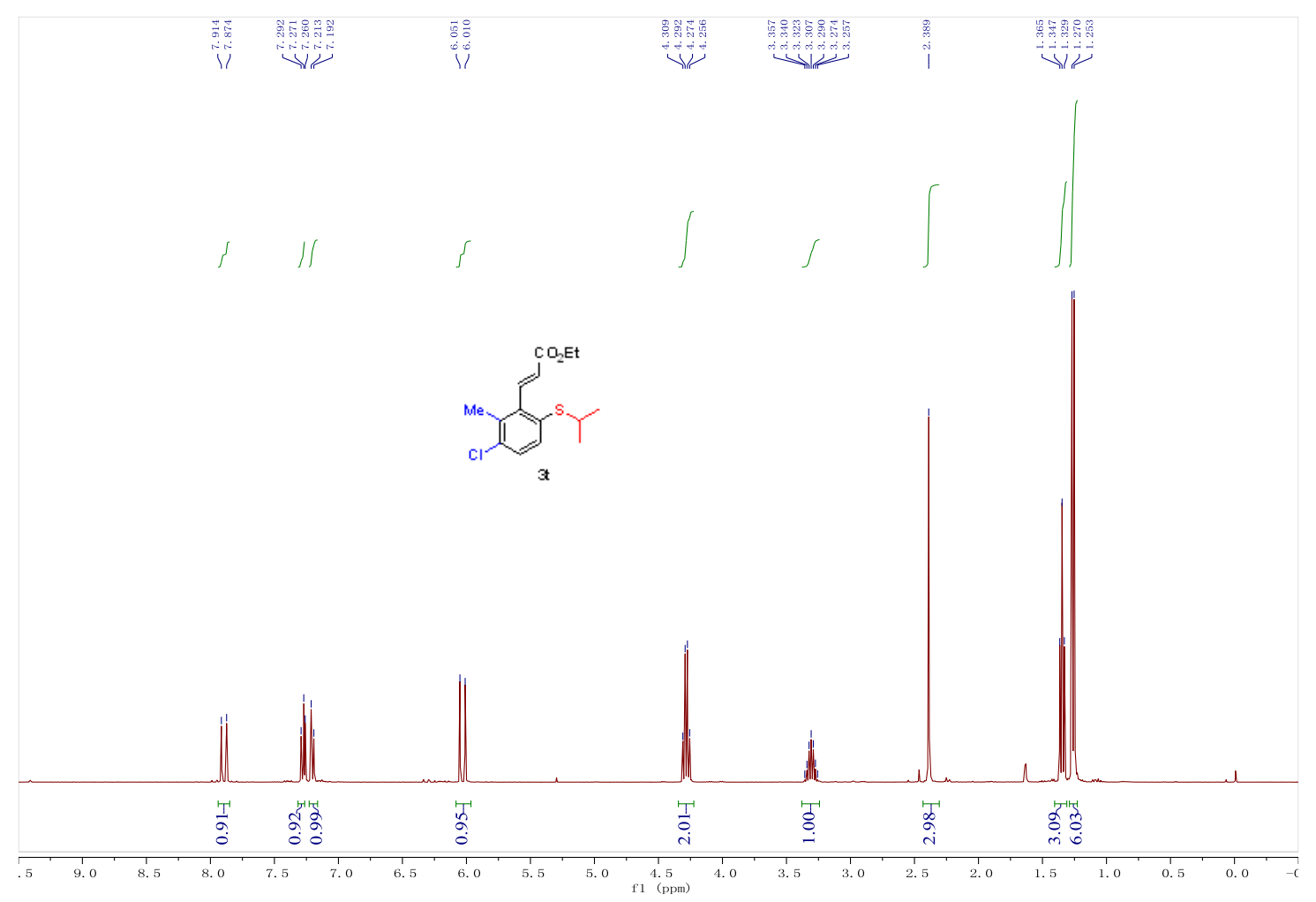

${ }^{1}$ H NMR
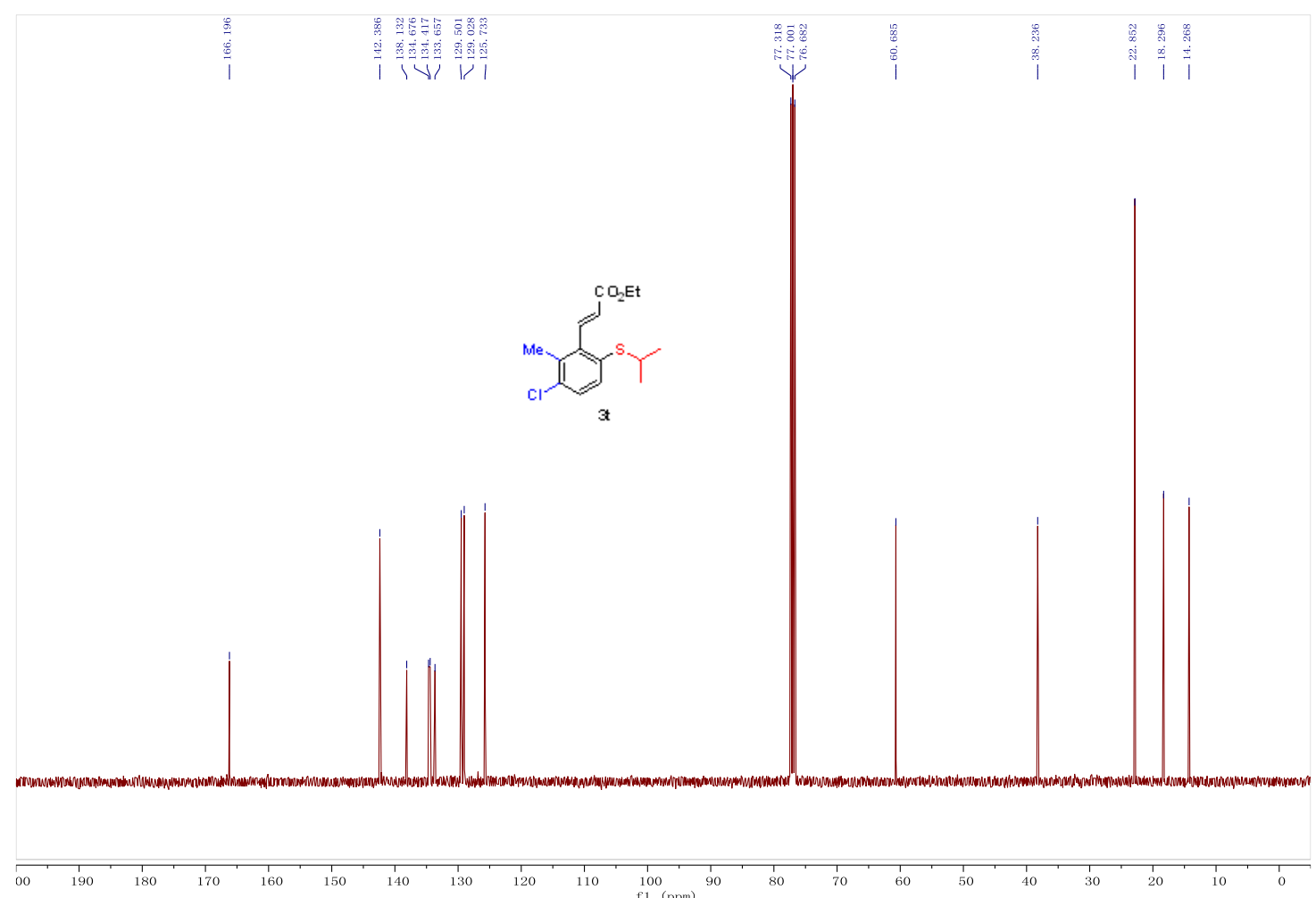

${ }^{13}$ C NMR 


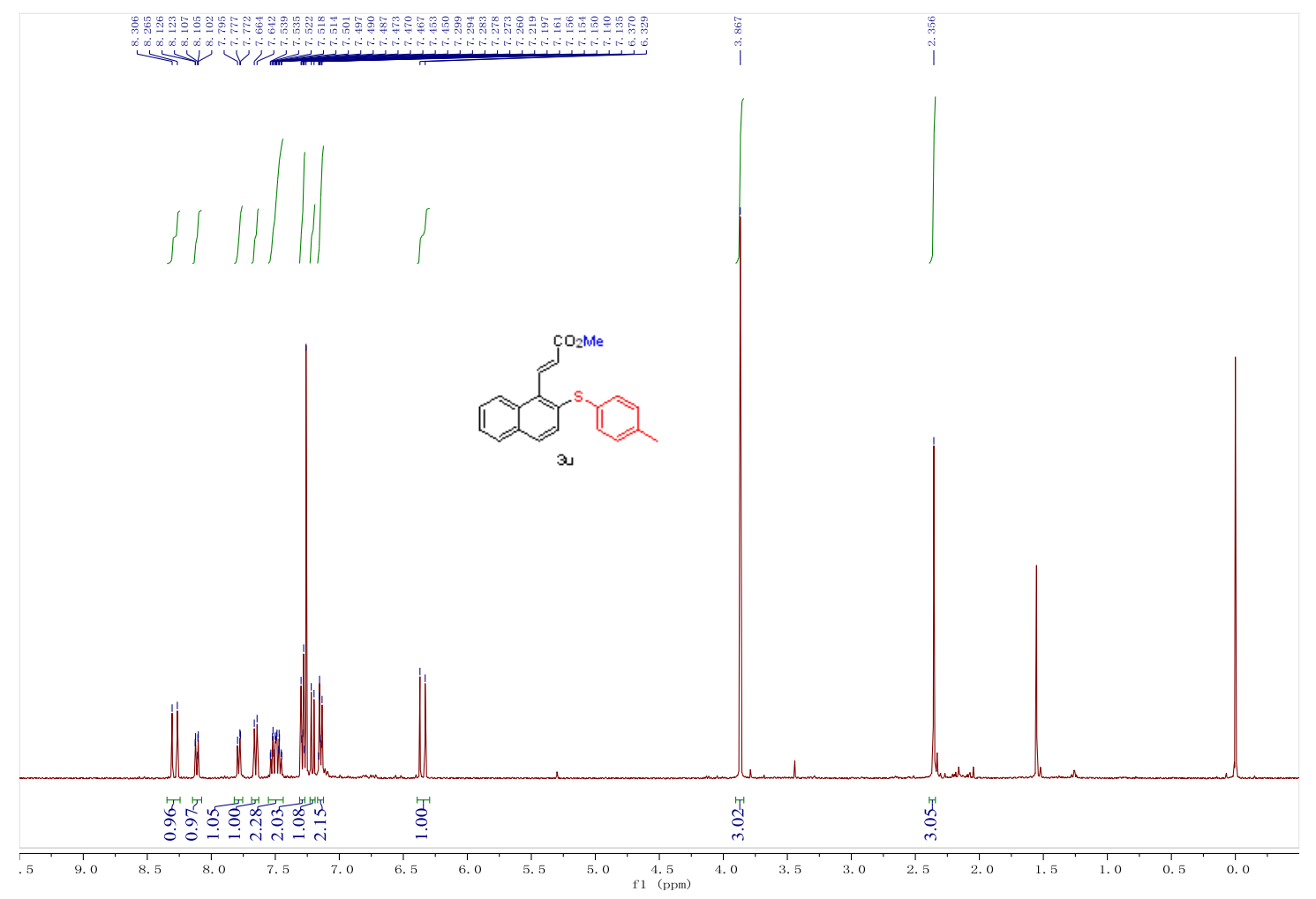

\section{${ }^{1}$ H NMR}
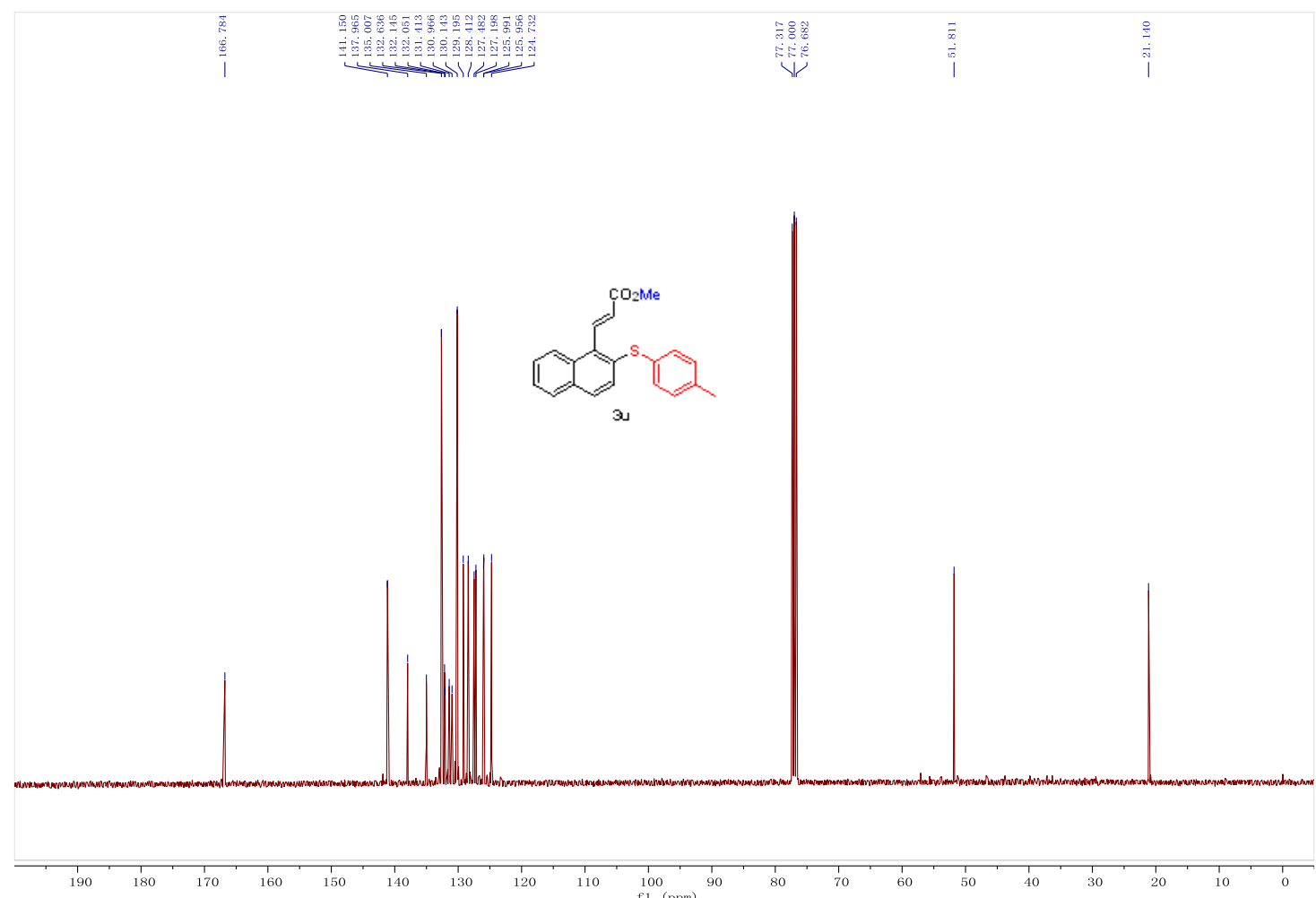

${ }^{13}$ C NMR 


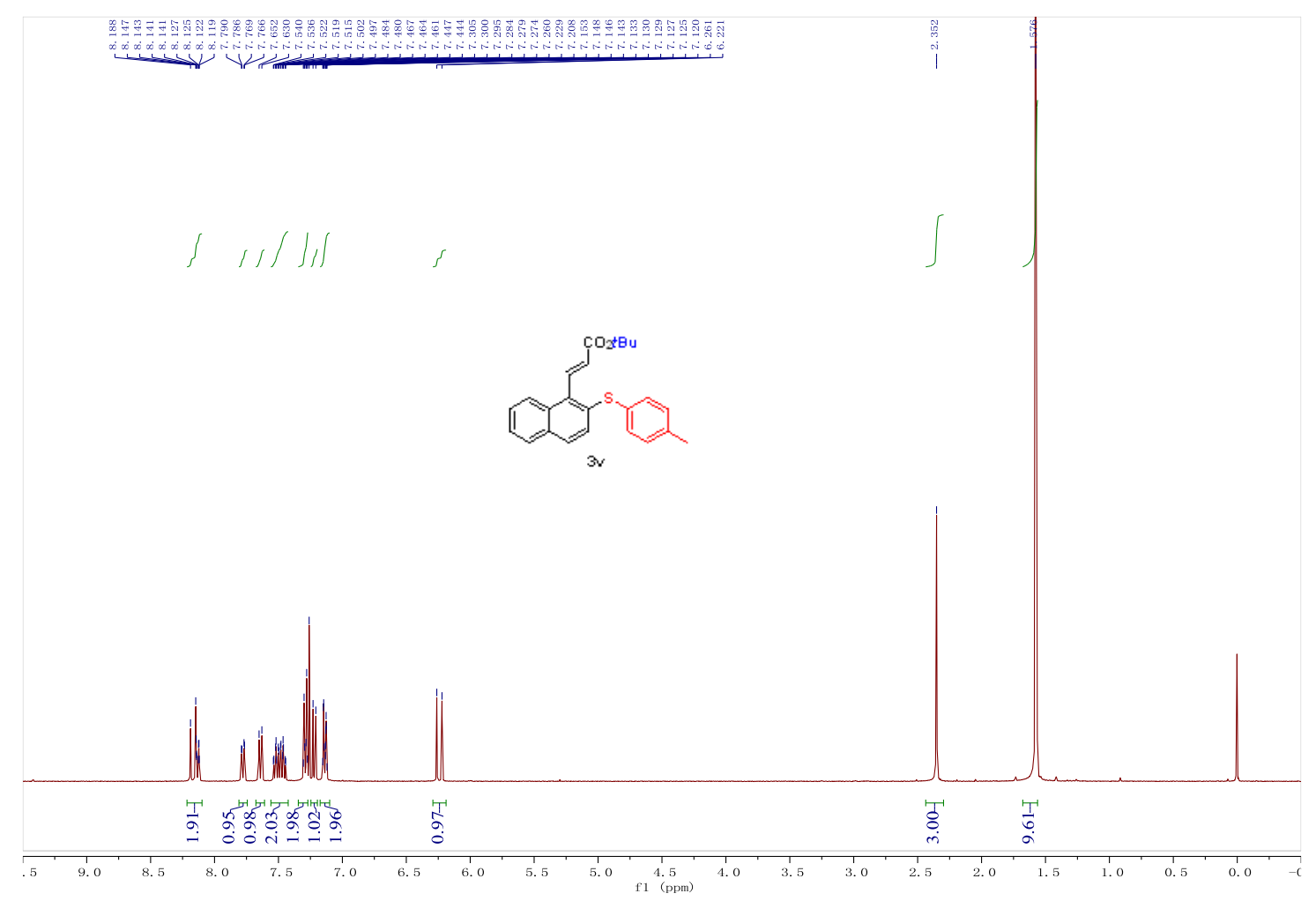

${ }^{1}$ H NMR
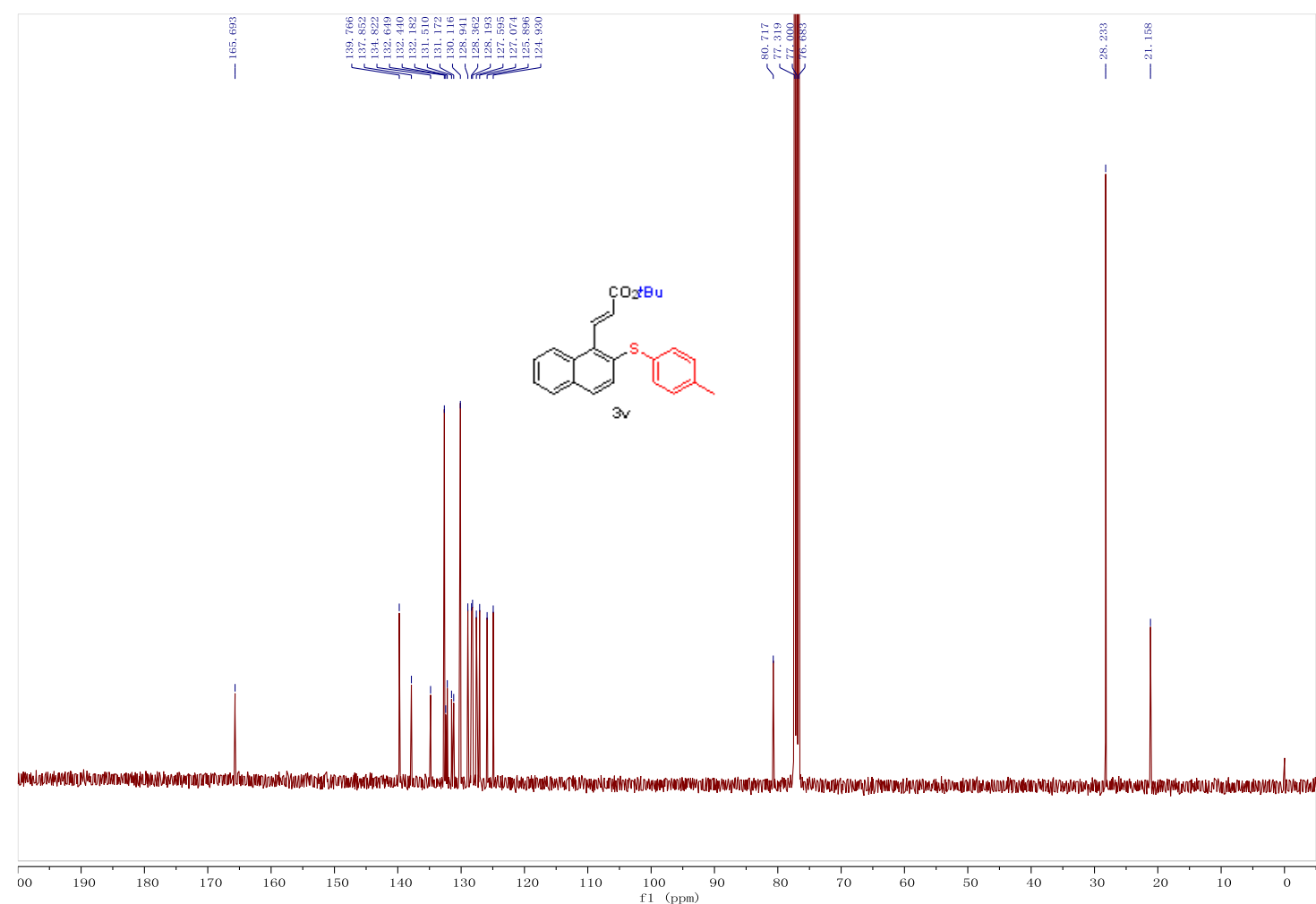

${ }^{13}$ C NMR 


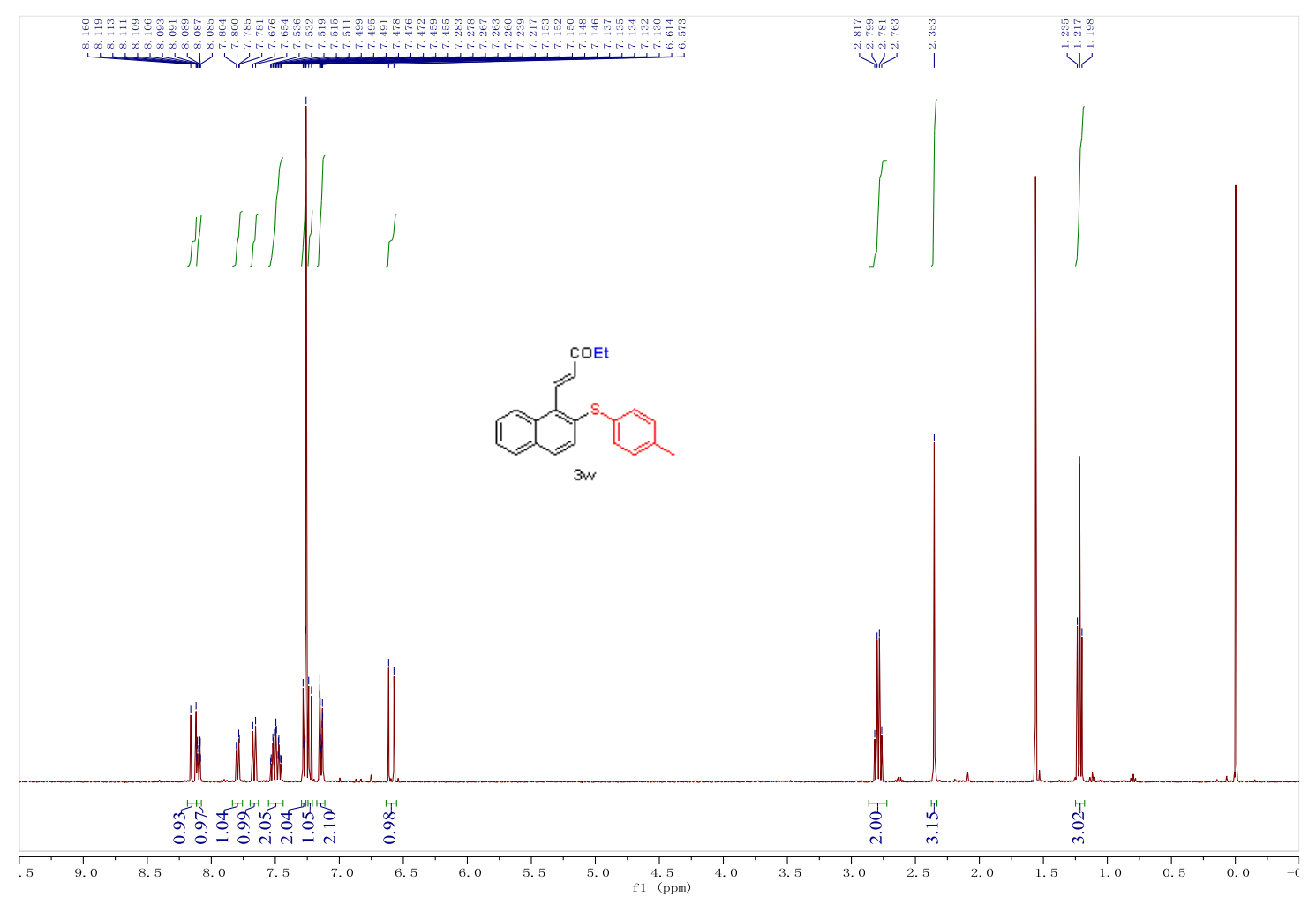

${ }^{1}$ H NMR

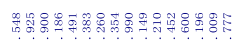

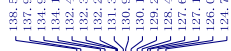
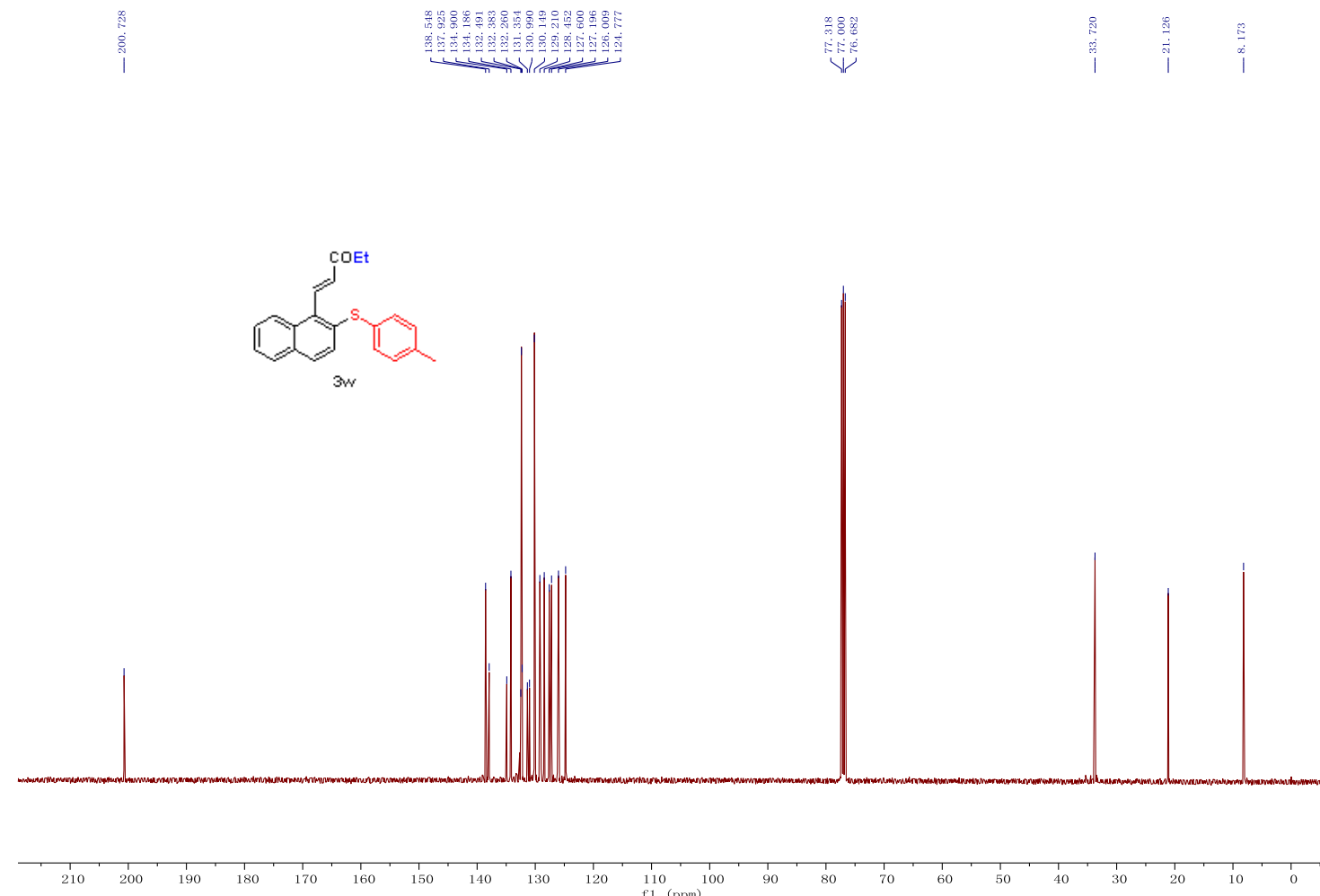

${ }^{13} \mathrm{C}$ NMR 


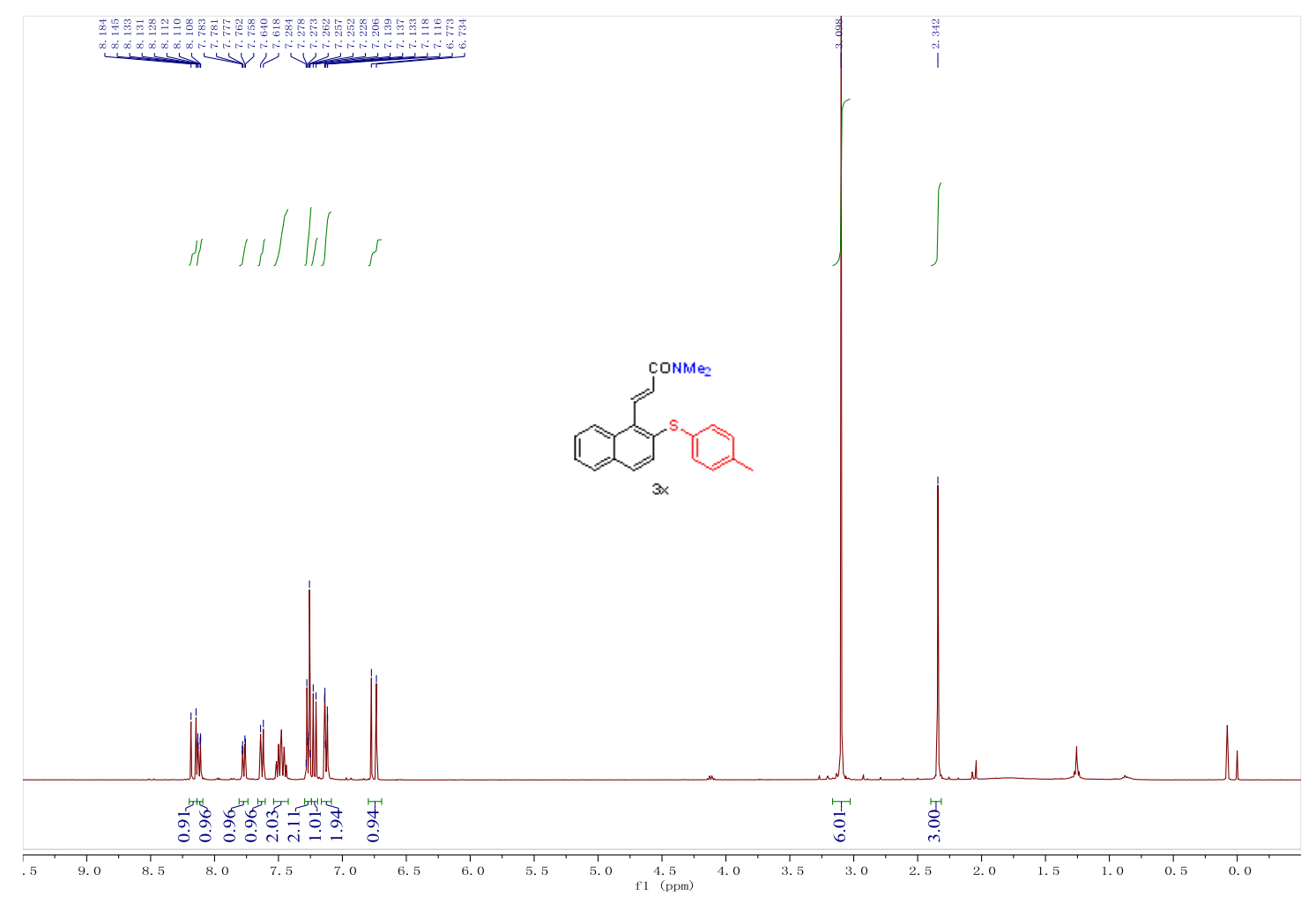

\section{${ }^{1}$ H NMR}
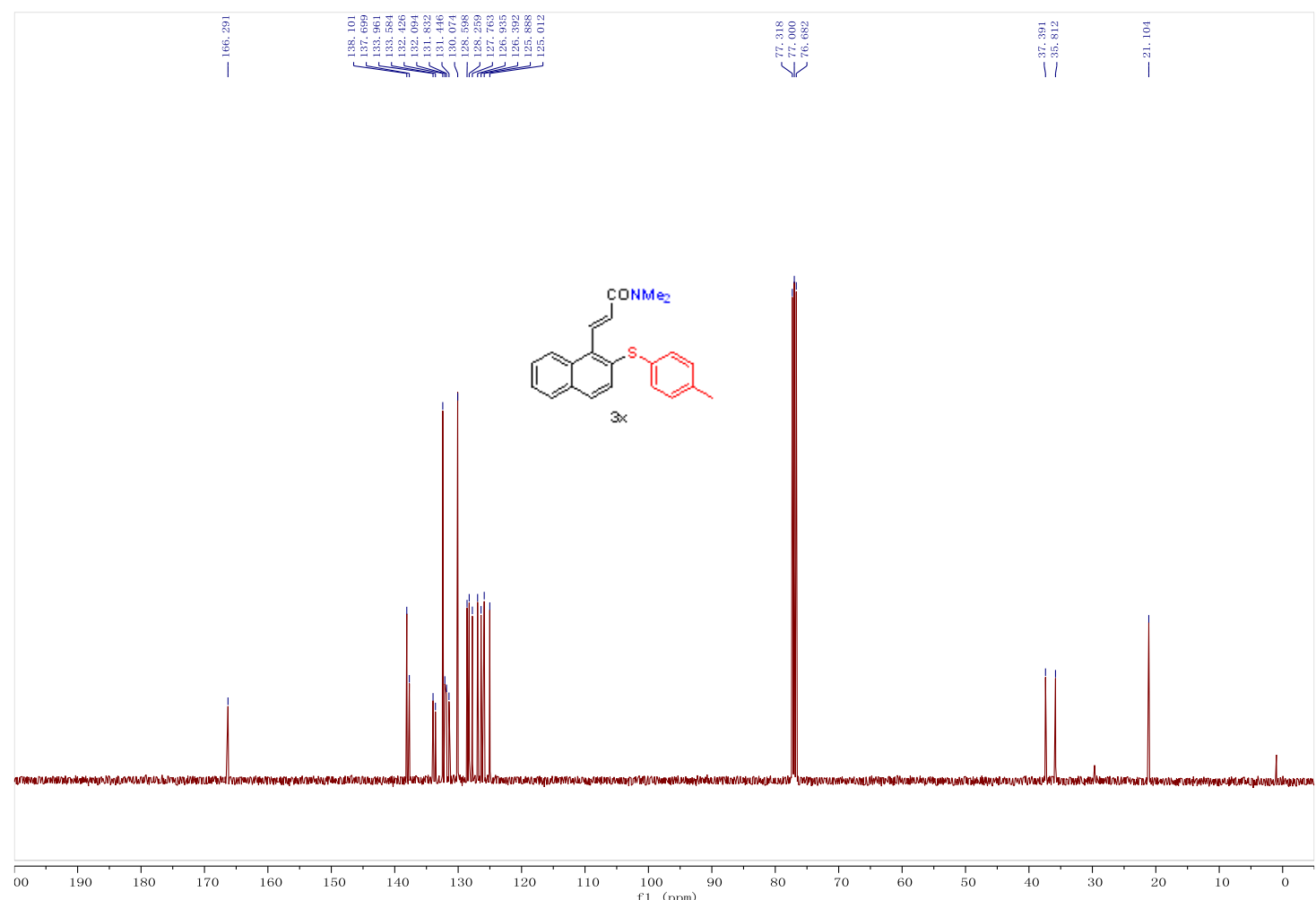

${ }^{13}$ C NMR 


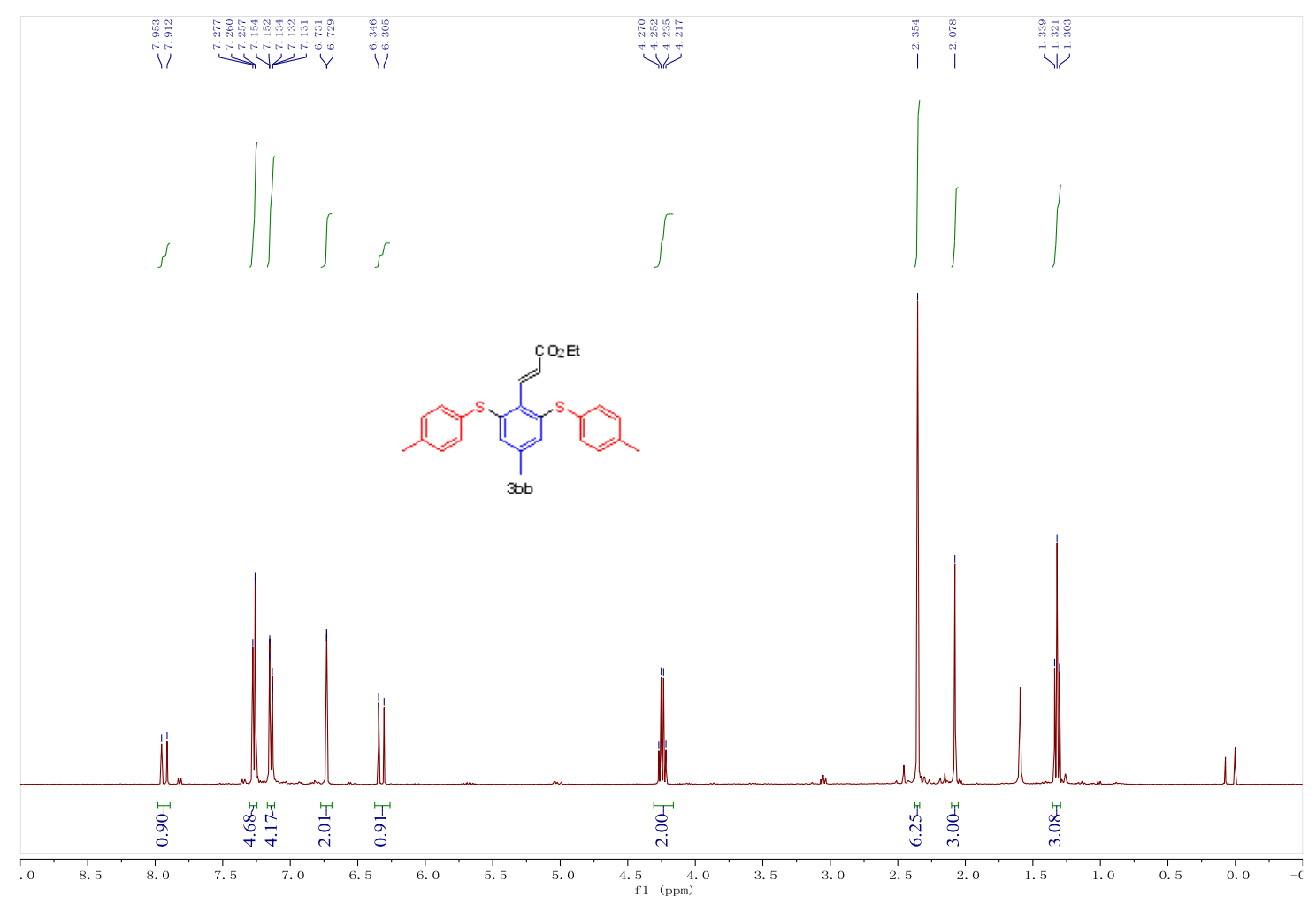

\section{H NMR}
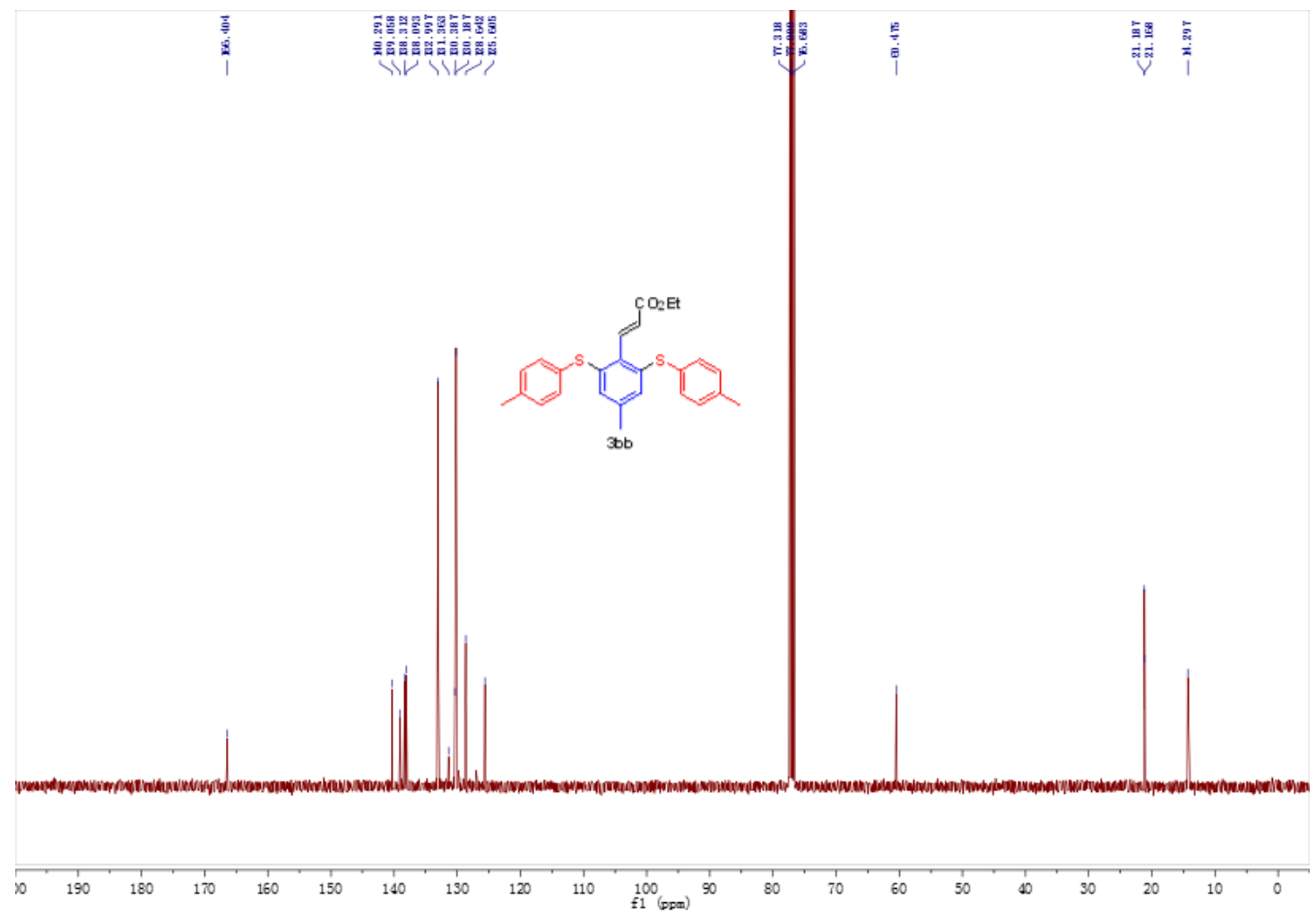

${ }^{13} \mathrm{C}$ NMR 


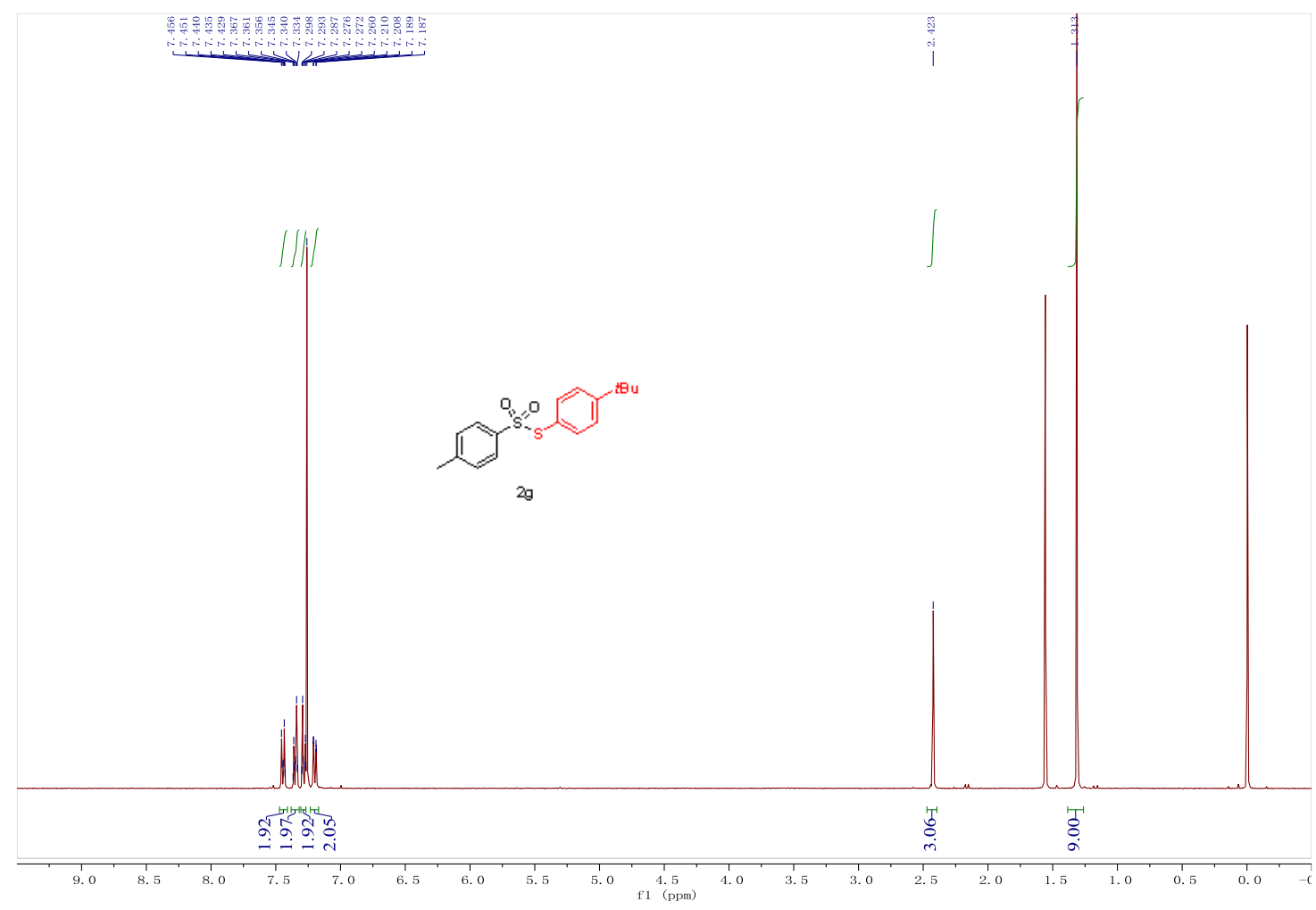

${ }^{1}$ H NMR
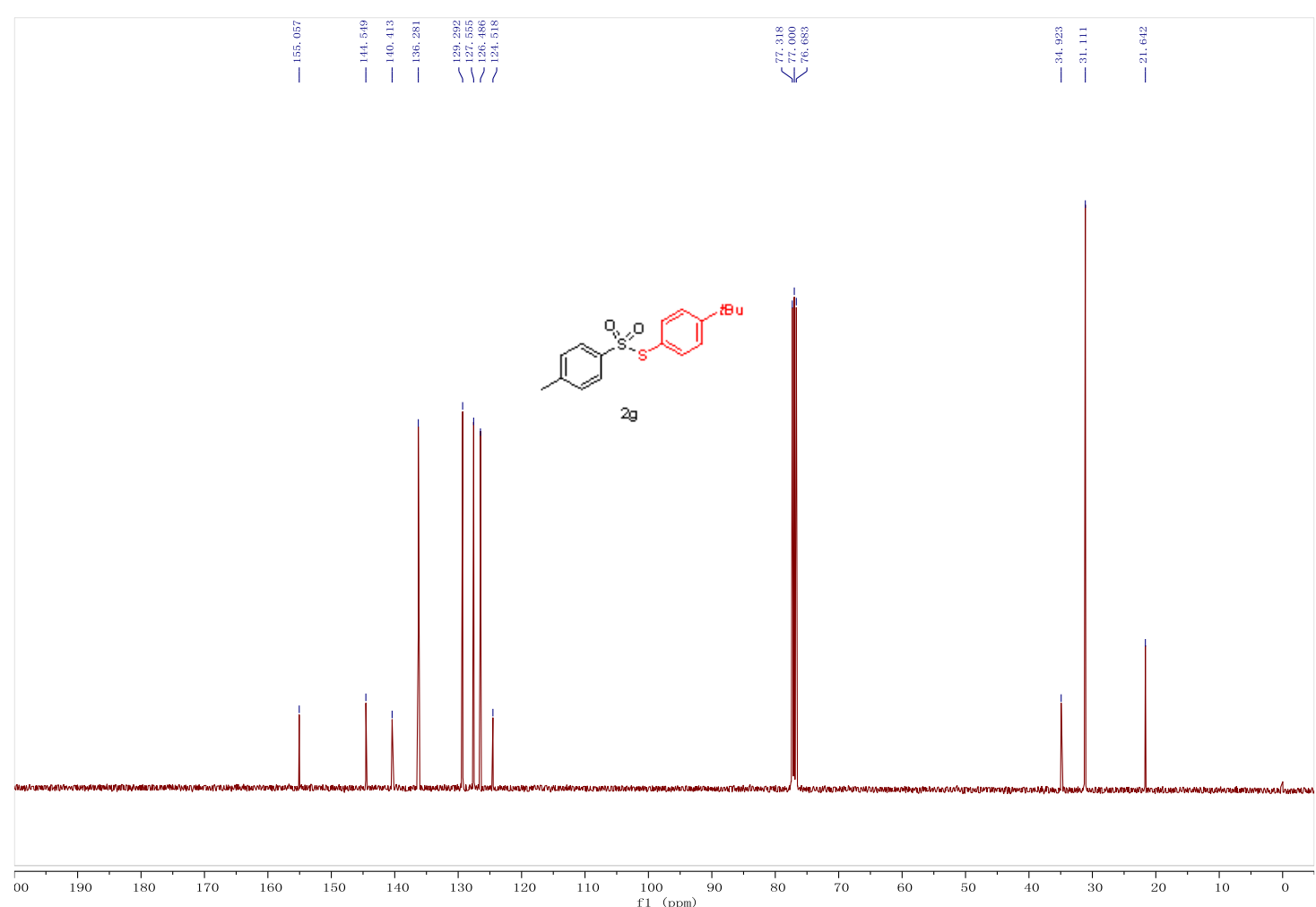

${ }^{13}$ C NMR 


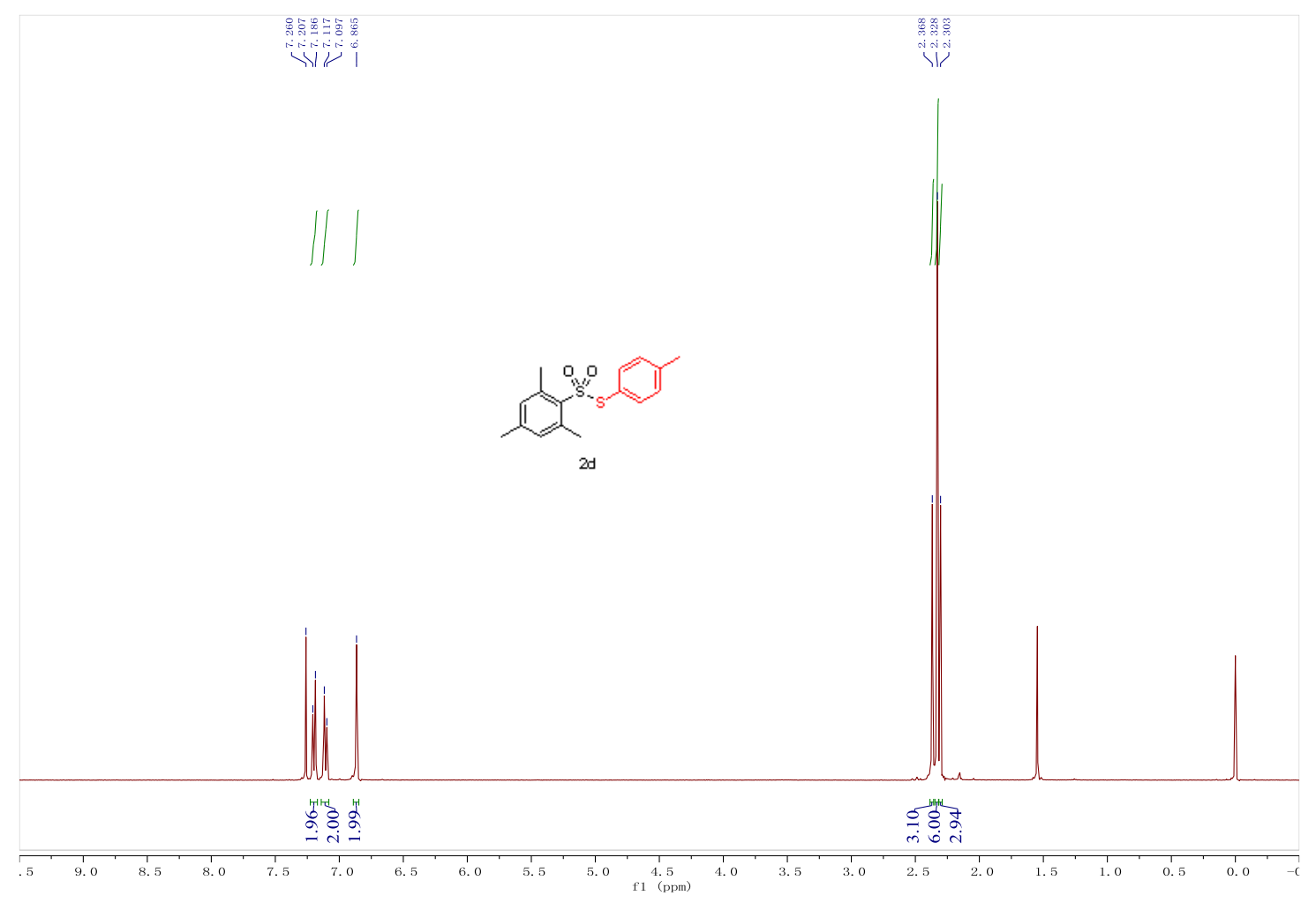

${ }^{1}$ H NMR
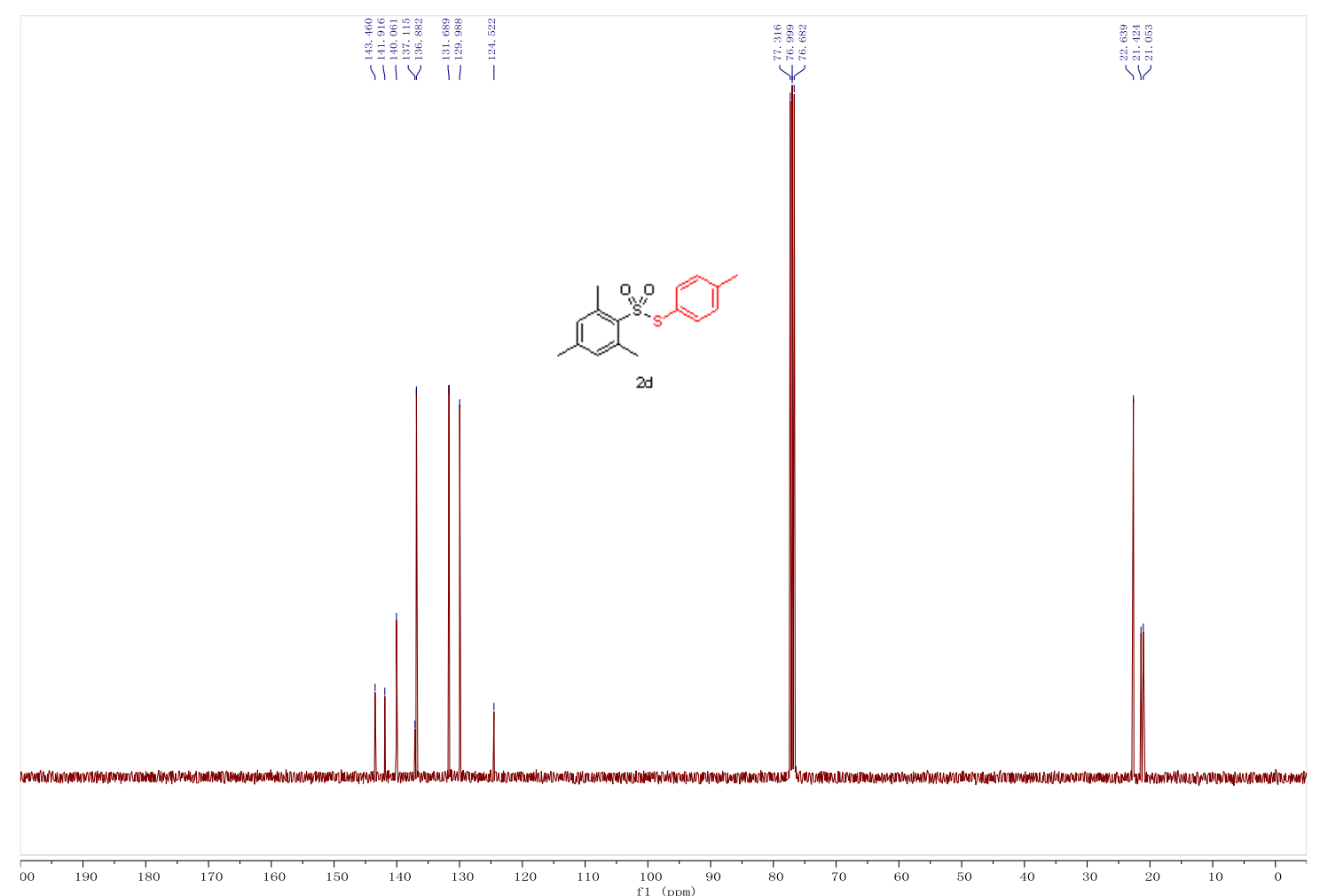

${ }^{13}$ C NMR 


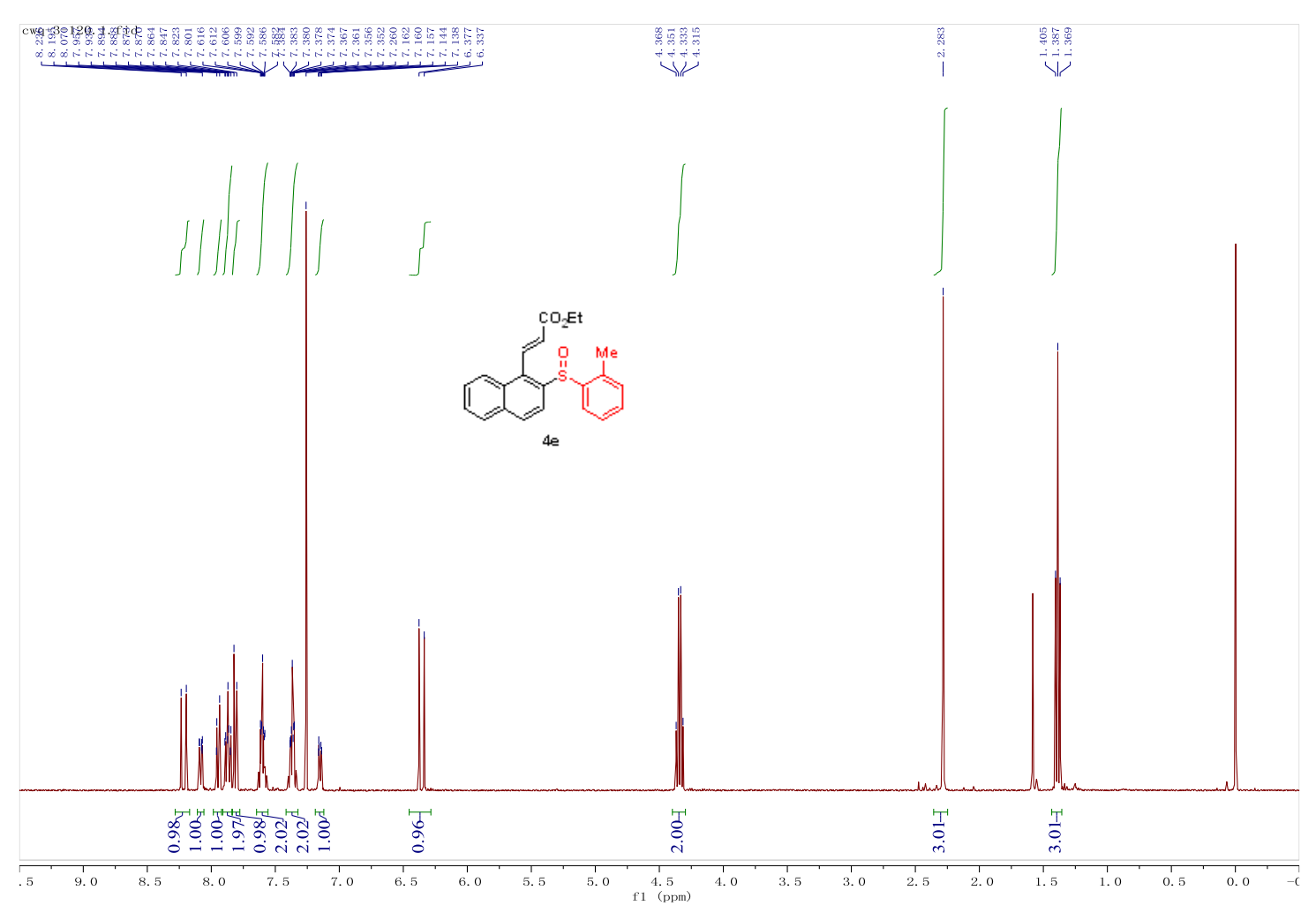

\section{${ }^{1}$ H NMR}
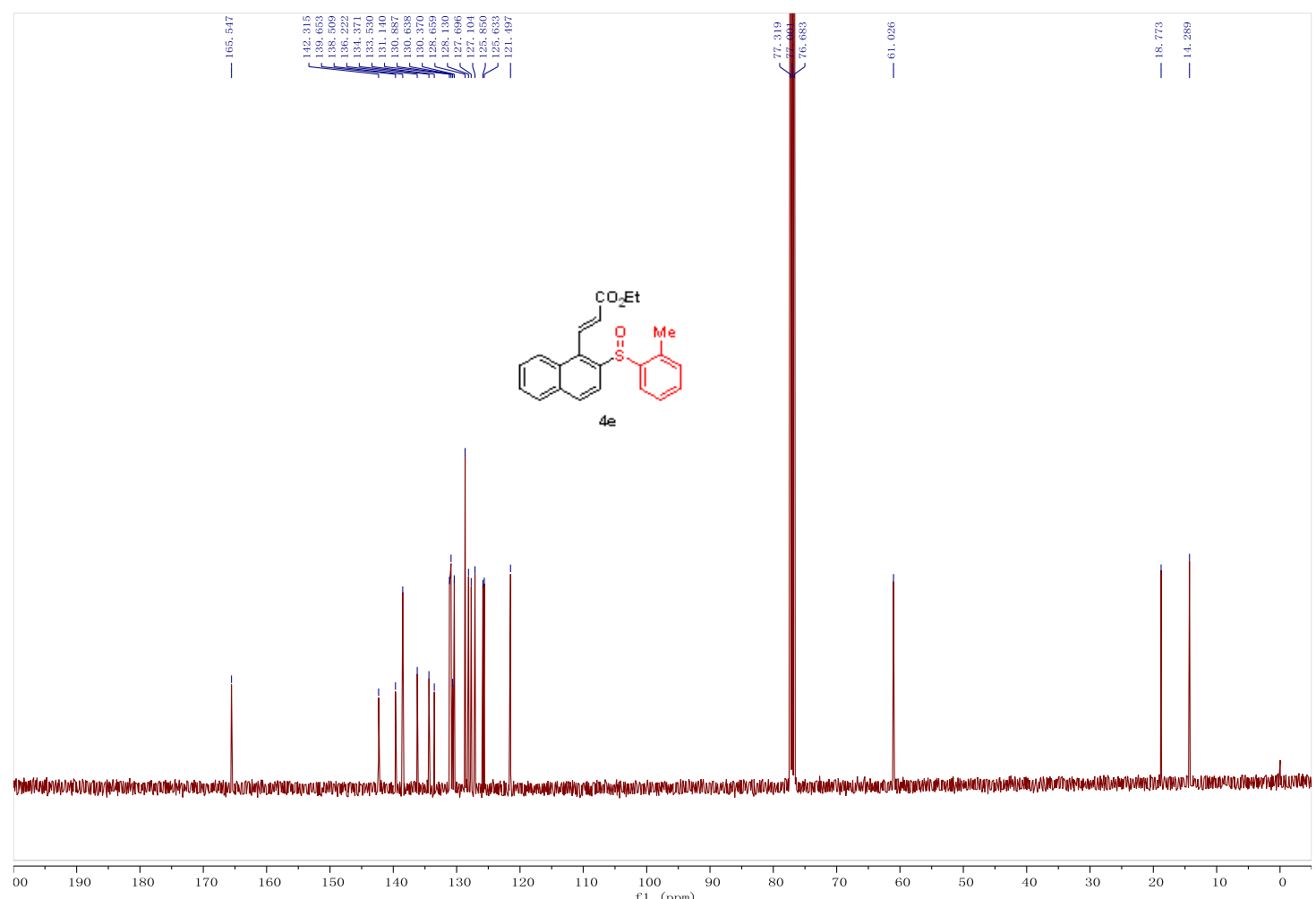

${ }^{13}$ C NMR 


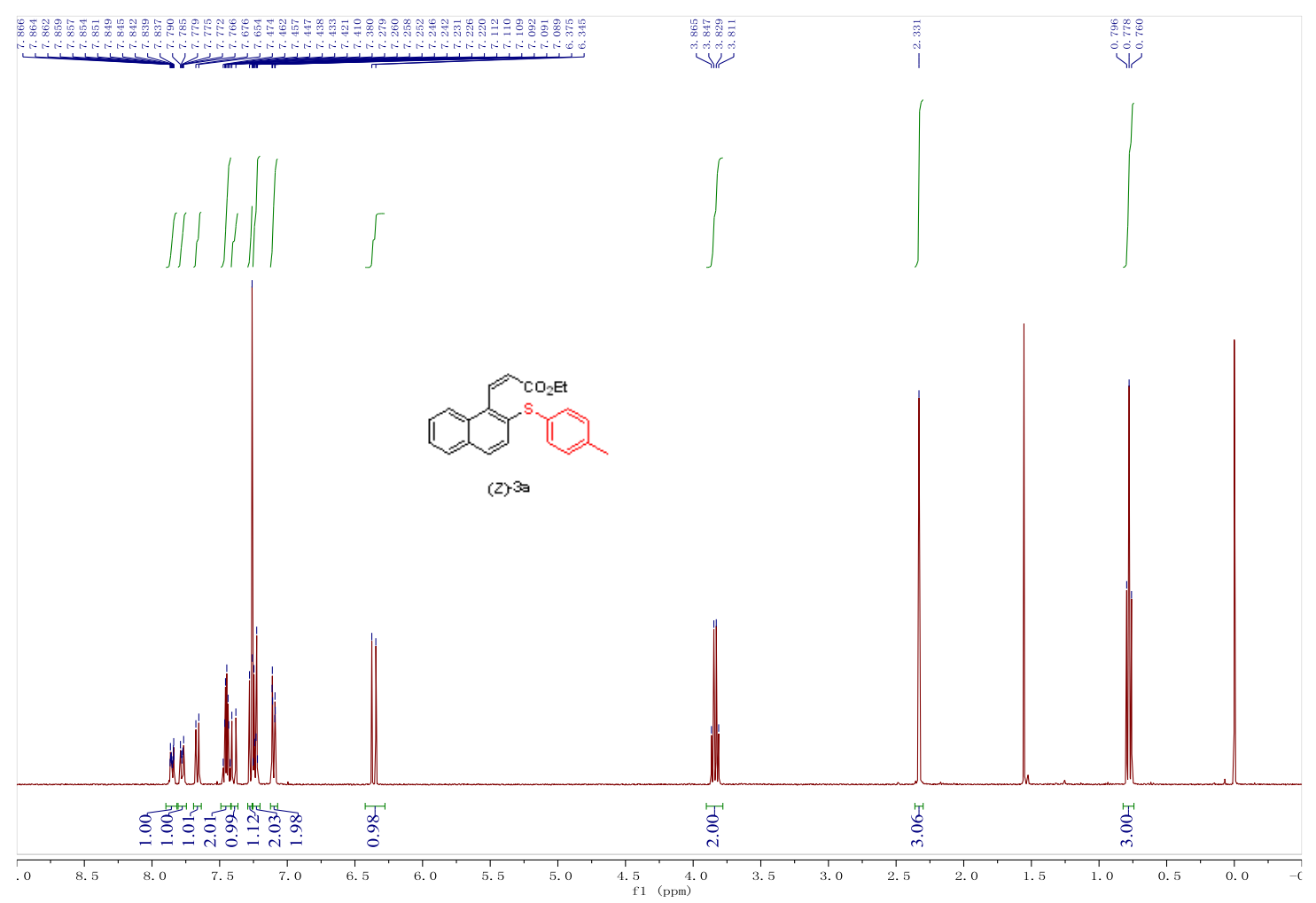

${ }^{1}$ H NMR

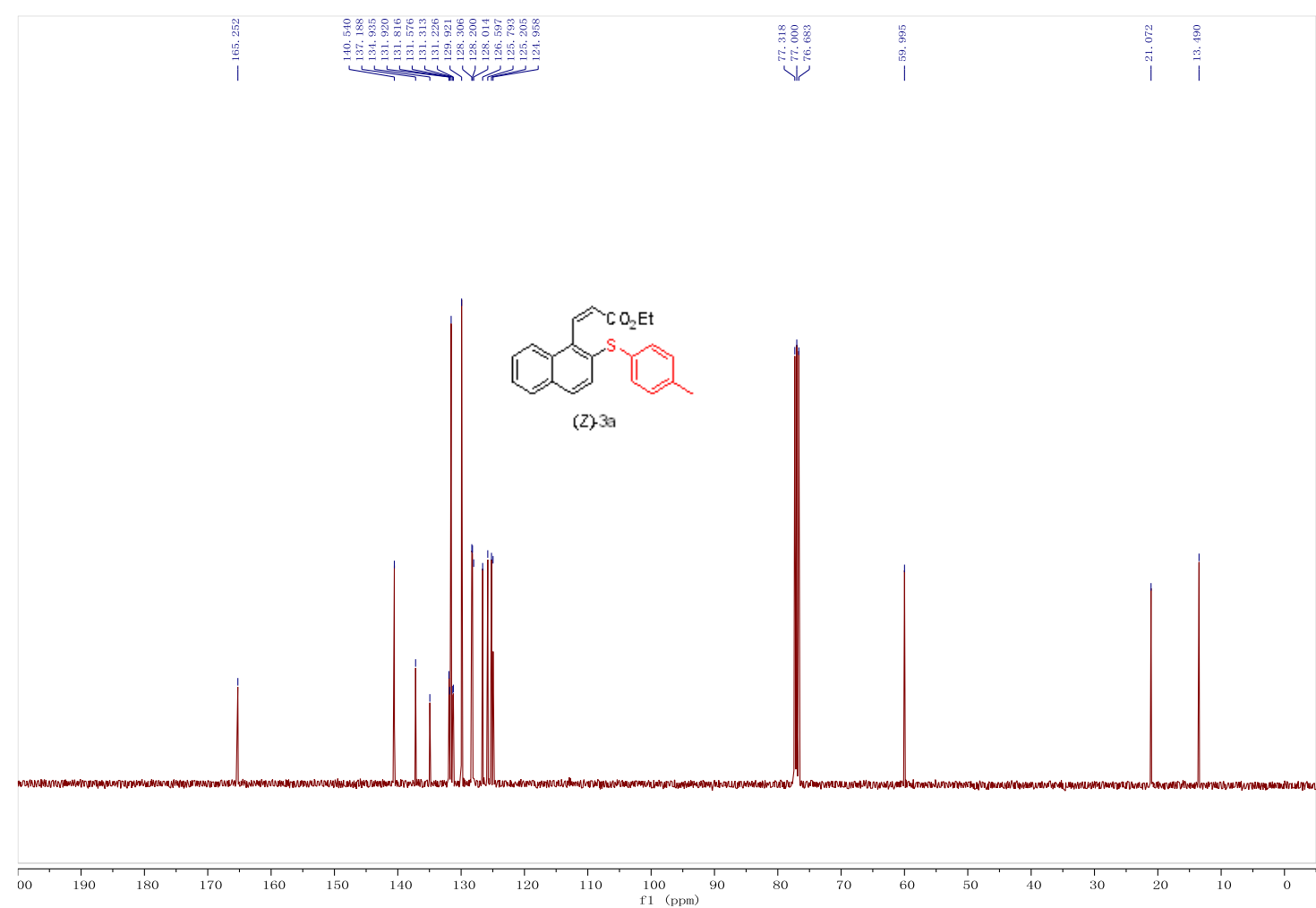

${ }^{13}$ C NMR 
III I

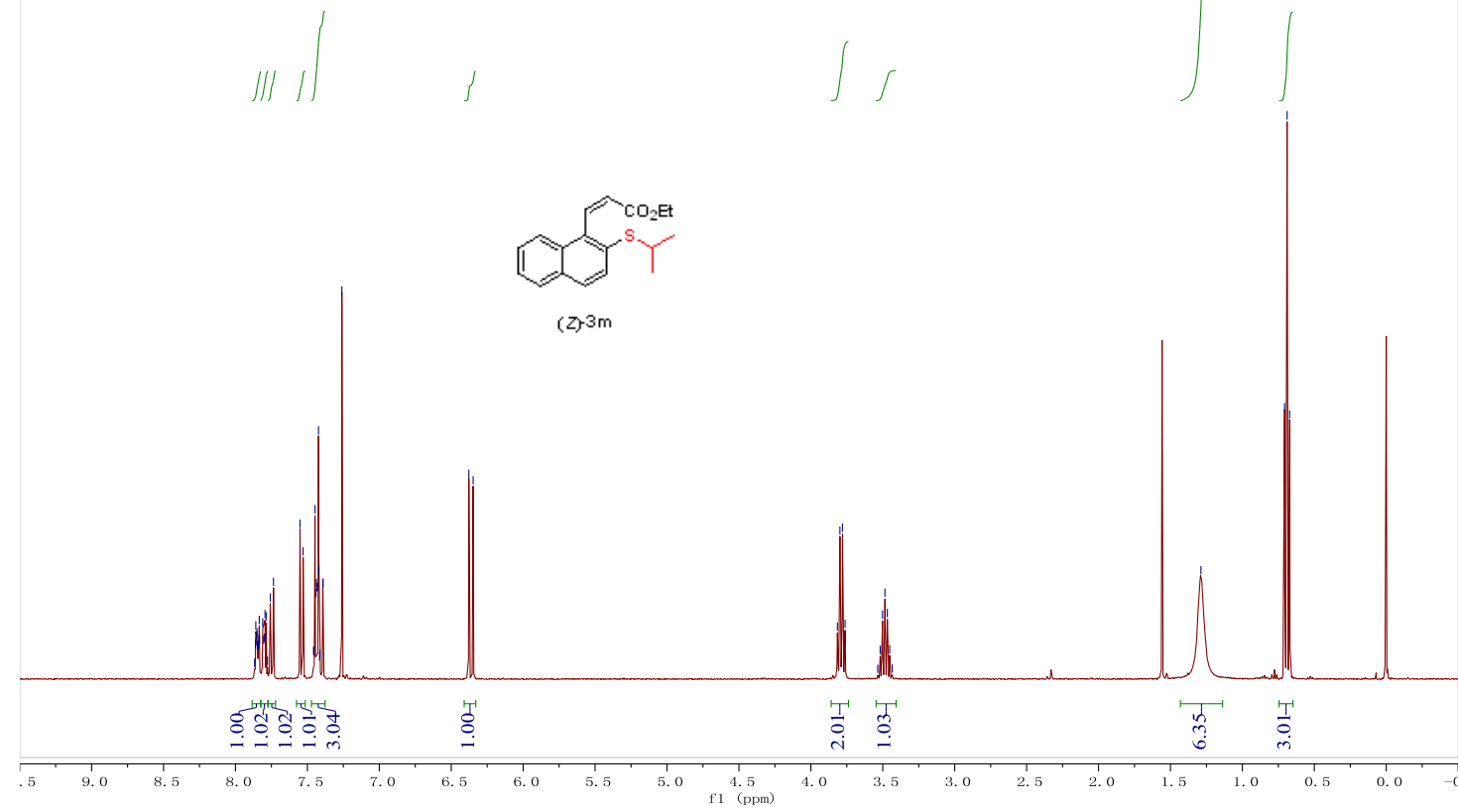

${ }^{1}$ H NMR
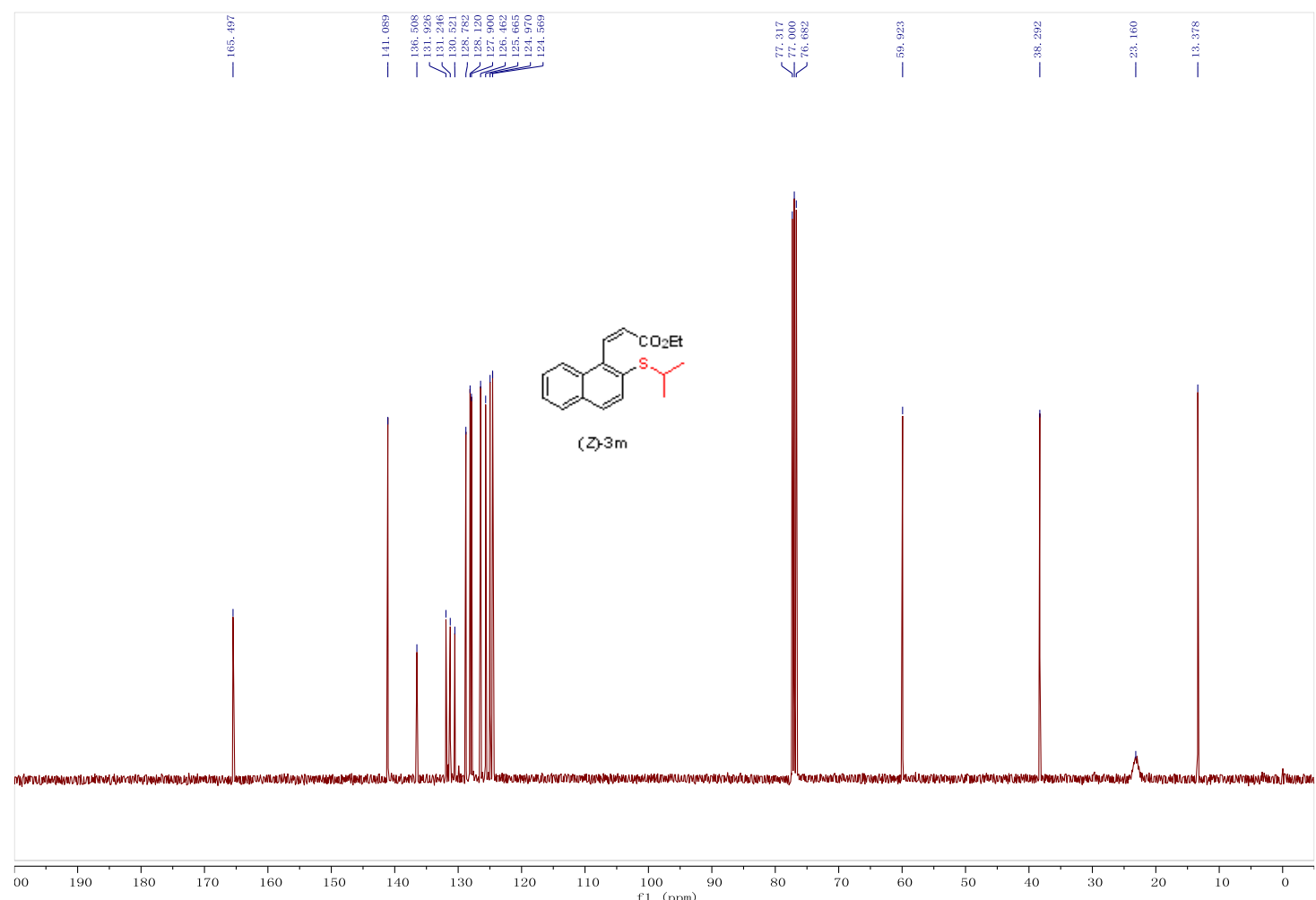

${ }^{13}$ C NMR 